\title{
Abstracts of the 20th College of Medicine Research Dissemination Conference
}

Theme: College of Medicine Research Excellence Yesterday, Today, and Tomorrow

MALARIA

\section{A1 A cost-effectiveness analysis of a school-based malaria intervention in Zomba District}

Linda A. Sande ${ }^{1,2}$, Levison S. Chiwaula ${ }^{2}$, Patrick Kambewa ${ }^{2}$, Don P. Mathanga ${ }^{3}$

1. Malawi-Liverpool-Wellcome Trust Clinical Research Programme, Blantyre, Malawi

2. Chancellor College, University of Malawi, Zomba, Malawi

3. Malaria Alert Centre, College of Medicine, University of Malawi,

Blantyre, Malawi

\section{Introduction}

Malaria is responsible for 50 percent of all preventable absenteeism and leads to a loss of 4 to 10 million school days per year among African school-going children. In Malawi, malaria is a frequent cause of absenteeism in school, resulting in poor scholastic performance among students. Since 2013, Save the Children International (SCI) has been implementing a malaria intervention in primary schools in Traditional Authority (TA) Chikowi, Zomba district. The intervention protocol includes malaria diagnosis using malaria rapid diagnostic tests (mRDTs) and treatment of uncomplicated cases using artemisinin combination therapy (ACT), with all of this carried out by teachers. We conducted a cost-effectiveness analysis of the school-based malaria intervention programme in TA Chikowi in order to determine if the strategy is economically viable.

\section{Methods}

The study modelled treatment-seeking behaviour using decision tree modelling, with costs analysed from a societal perspective using the ingredients method. Effectiveness was measured as expected school attendance days and cost-effectiveness was assessed using an incremental cost-effectiveness ratio, with outpatient malaria care as the comparator.

\section{Results}

The TA Chikowi intervention strategy is highly cost-effective, with the opportunity cost of teachers' time, antimalarial drugs, and staff costs being the cost drivers. The annual cost of the intervention is US $\$ 12.57$ (MWK5,781.58) per child, which (for each additional schooling day gained) is US\$0.28 (MWK128.81) more than it costs for a health facility to deliver outpatient malaria services in the same time period.

\section{Conclusions}

The intervention should be implemented and scaled-up to other areas in the district and is expected to be highly cost-effective in areas with high malaria prevalence.

\section{A2 Assessing the spatial heterogeneity of malaria vectors in the context of increasing vector control interventions}

$\underline{\text { Robert S. McCann }}{ }^{1,2}$, Monicah M. Mburu ${ }^{1}$, Daniela Schluter ${ }^{3}$, Michael G. Chipeta ${ }^{3}$, Peter J. Diggle ${ }^{3}$, Dianne J. Terlouw ${ }^{4}$, Henk van den Berg ${ }^{1}$, Kamija Phiri' ${ }^{2}$, Michèle van Vugt ${ }^{5}$, Willem Takken ${ }^{1}$

1. Wageningen University \& Research, Wageningen, the Netherlands

2. College of Medicine, University of Malawi Blantyre, Malawi
3. Lancaster University, Lancaster, United Kingdom

4. Liverpool School of Tropical Medicine, Liverpool, United Kingdom 5. University of Amsterdam, Amsterdam, the Netherlands

\section{Introduction}

Environmental variation across a landscape naturally contributes to spatial heterogeneity in malaria risk, yet the coverage of malaria interventions is increasing dramatically across many endemic regions. Novel geostatistical methods to investigate the spatiotemporal heterogeneity of malaria are increasingly available in opensource software. Investigating the spatio-temporal heterogeneity of malaria vectors is critical for understanding how transmission patterns change in the context of increasing intervention coverage.

\section{Methods}

In this study we investigated the spatio-temporal heterogeneity of host-seeking adult mosquitoes in a region of southern Malawi where malaria interventions are being intensively scaled-up. We used geostatistical methods to efficiently sample houses for mosquitoes at a sub-district level. Host-seeking mosquitoes were collected using Suna traps on a continuous, rolling basis for one year. We used model-based geostatistics to account for spatial correlation in assessing the determinants of mosquito distribution, and to map spatial variation in the abundance of mosquitoes.

\section{Results}

Preliminary findings suggest clear spatial patterns in mosquito abundance.

\section{Conclusions}

The results provide a basis for determining the spatio-temporal heterogeneity of malaria in the context of a community-based, vector-control intervention trial.

\section{A3 Risk factors for Anopheles spp. mosquitoes in rural and urban areas in Blantyre District, southern Malawi}

Themba Mzilahowa ${ }^{1}$, Madalitso Luka-Banda ${ }^{1}$, Veronica Uzalili ${ }^{1}$, Don P. Mathanga ${ }^{1}$, Carl H. Campbell $\mathrm{Jr}^{2}$, Mavuto Mukaka ${ }^{3}$, John E. Gimnig ${ }^{4}$

1. Malaria Alert Centre, College of Medicine, University of Malawi, Blantyre, Malawi

2. Center for Tropical and Emerging Global Diseases, University of Georgia, Athens, Georgia, USA

3. Malawi-Liverpool-Wellcome Trust Clinical Research Programme, Blantyre, Malawi

4. Entomology Branch, Division of Parasitic Diseases and Malaria, Centers for Disease Control and Prevention, Atlanta, Georgia, USA

\section{Introduction}

Although urban malaria transmission is low and characteristically seasonal, it continues to be a major public health problem. This study aimed at demonstrating the presence of Anopheles mosquitoes and the potential for malaria transmission in urban settings.

\section{Methods}

Two cross-sectional surveys were carried out in Blantyre District, covering both urban and rural areas during the wet and dry seasons of 2008 and 2010, respectively. A grid was laid over Blantyre and 60 cells were randomly selected. Five households located within 100 $\mathrm{m}$ from the centroid of each selected cell were enrolled, a standard questionnaire was administered, and indoor resting mosquitoes were sampled using backpack aspirators. 


\section{Results}

In 2008, a total of 960 mosquitoes were collected of which $9.9 \%$ ( $\mathrm{n}=95$ ) were morphologically identified as An. funestus and bulk were culicines. A subsample $(\mathrm{n}=14)$ were successfully identified as An. funestus s.s. by PCR. In the wet season, 1045 mosquitoes were captured and $A n$. funestus remained the predominant anopheline $(10.3 \% ; \mathrm{n}=108)$. All the Anopheles mosquitoes were collected from households located in rural areas of Blantyre and none from urban areas. In univariate analysis, open eaves was associated with increased Anopheles, both during dry (incidence rate ratio [IRR] = 4.3; $95 \%$ CI 2.4 to 7.6 ) and wet (IRR $=2.47 ; 95 \%$ CI 1.7 to 3.59 ) seasons. Chances of detecting Anopheles decreased with increasing altitude (IRR $=0.996 ; 95 \%$ CI 0.995 to 0.997 ) during the dry season but increased during the wet season $($ IRR $=1.0017 ; 95 \%$ CI 1.0012 to 1.0023). These factors remained significant following a multiple Poisson regression analysis. No association was found between ITN ownership and the number of Anopheles mosquitoes.

\section{Conclusions}

The presence of An. funestus s.s and An. gambiae s.l. in the periphery of Blantyre city was an indication that malaria transmission was potentially taking place in these areas.

\section{A4 Identifying malaria hotspots through continuous malaria prevalence surveys using adaptive sampling design}

$\underline{\text { Alinune N. Kabaghe }}^{1,2}$, Michael G. Chipeta ${ }^{2,3}$, Robert S. McCann ${ }^{2,5}$, Kamija S. Phiri ${ }^{2}$, Michele van Vugt ${ }^{1}$, Willem Takken ${ }^{5}$ Peter Diggle ${ }^{3}$, Dianne J. Terlouw ${ }^{4}$

1. Academic Medical Center, University of Amsterdam, Amseterdam, the Netherlands

2. College of Medicine, University of Malawi, Blantyre, Malawi

3. Lancaster University, Lancaster, United Kingdom

4. Malawi-Liverpool-Wellcome Trust Clinical Research Programme,

Blantyre, Malawi

5. Wageningen University \& Research, Wageningen, the Netherlands

\section{Introduction}

Diseases with spatial and temporal variations need to be accurately mapped for targeted interventions. Existing sampling strategies and surveys for disease prevalence lack the efficiency to detect fine scale spatial heterogeneity, temporal variations, and disease hotspots. Continuous monitoring of disease burden readily provides visualization of local spatio-temporal variation. Adaptive sampling design improves prediction of outcome of interest compared to current random sampling technique. We present findings of continuous malaria prevalence surveys using an adaptive sampling design.

\section{Methods}

We conducted repeated cross sectional surveys guided by an adaptive sampling design to monitor the prevalence of malaria parasitaemia in children 6-59 months old within the Majete Malaria Project in Chikwawa district, Southern Malawi. We fitted a geostatistical model to predict malaria prevalence in the area. The Majete Malaria Project is funded by Dioraphte Foundation, the Netherlands.

\section{Results}

We conducted five sampling rounds and tested 876 children aged 6-59 months from 1377 households over a 12 month period. Malaria prevalence prediction maps showed fine scale spatial heterogeneity and presence of hotspots in the study area. Predictors of malaria include age, social economic status, and ownership of insecticide treated mosquito nets.

\section{Conclusions}

Continuous malaria prevalence surveys using adaptive sampling increased malaria prevalence prediction accuracy. Results from the surveys were readily available after data collection. The approach can assist local managers to target malaria control interventions in areas with the greatest health impact and is readily applicable to other diseases with spatial heterogeneity.

\section{A5 Patterns and determinants of malaria risk in urban and peri-urban areas of Blantyre, Malawi}

\section{Don P. Mathanga ${ }^{1,2}$, Atupele Kapito Tembo ${ }^{1}$, Themba}

Mzilahowa ${ }^{1}$, Andy Bauleni ${ }^{1}$, Kondwani Mtimaukenena ${ }^{1}$, Terrie E. Taylor ${ }^{3}$, Clarissa Valim ${ }^{4}$, Edward Walker ${ }^{5}$, Mark L. Wilson ${ }^{6}$

1. Malaria Alert Centre, College of Medicine, University of Malawi, Blantyre, Malawi

2. Department of Community Health, School of Public Health and Family Medicine, College of Medicine, University of Malawi, Blantyre, Malawi

3. College of Osteopathic Medicine, Michigan State University, East Lansing, Michigan, USA

4. Department of Immunology and Infectious Diseases, Harvard School of Public Health, Boston, Massachusetts, USA

5. Department of Microbiology and Molecular Genetics, Michigan State University, East Lansing, MI, USA

6. Department of Epidemiology, School of Public Health University of Michigan, Ann Arbor, Michigan, USA

\section{Introduction}

Although malaria disease in urban and peri-urban sub-Saharan Africa is a growing concern, the epidemiologic patterns and drivers of transmission in these settings remain poorly understood. In this study, factors associated with variation in malaria risk in urban and peri-urban areas of Blantyre, Malawi were evaluated.

\section{Methods}

A health facility-based, case-control study of children 6-59 months of age was conducted in four urban and two peri-urban facilities of Blantyre city. Cases and matched controls were defined as children who presented with malaria symptoms at the same clinic and tested positive or negative, respectively, for malaria parasites by microscopy and PCR. Controls were matched with cases for age, location (zones) and time of recruitment.

\section{Results}

A total of 187 cases and 286 controls were studied. In univariate analyses, higher level of education, possession of TV, and household electricity were associated with decreased malaria in both urban and peri-urban zones. Having travelled and slept away from home prior to the clinic visit also was associated increased malaria risk $(\mathrm{OR}=2.35 ; 95 \% \mathrm{CI}=1.04,5.3)$, however only for cases living in the urban zones $(\mathrm{OR}=5.1 ; 95 \% \mathrm{CI}=1.62,15.8)$. Use of insecticide treated bed nets the previous night was not associated with clinical malaria. In multivariate analyses, electricity, travel, and higher level education were all associated with odds of clinical disease. Few Anopheles mosquitoes were found inside households in the peri-urban areas, while none were collected from the urban households.

\section{Conclusions}

Overnight travel was the main factor influencing malaria illness in urban Blantyre but this association was not found in the periurban area. More detailed travel information and behaviour of the risk groups may help to more effectively control malaria in urban settings. 
A6 Dissecting the immunological basis of concurrent malaria and invasive bacterial infections in Malawian children

T.S. Nyirenda ${ }^{1,2}$, J.T. Nyirenda ${ }^{1,2}$, D. Tembo ${ }^{2}$, J. Storm ${ }^{2}$, Q. Dube $^{5}$, C. Msefula ${ }^{1}$, C.A. MacLennan ${ }^{4}$, R.S. Heyderman ${ }^{2,5}$, M.A. Gordon $^{2}$, W.L. Mandala ${ }^{2,6}$

1.Pathology Department, College of Medicine, University of Malawi, Blantyre, Malawi

2.Malawi-Liverpool-Wellcome Trust Clinical Research Programme,

Blantyre, Malawi

3. Department of Paediatrics and Child Health, Queen Elizabeth Central Hospital, Blantyre, Malawi

4. The Jenner Institute, Nuffield Department of Medicine, University of Oxford, Oxford, United Kingdom

5. University College London, London, United Kingdom

6. Basic Medical Sciences Department, College of Medicine, University of Malawi, Blantyre, Malawi

\section{Introduction}

Malaria is commonly associated with invasive nontyphoidal Salmonella (iNTS) disease in African children. Case fatality rate is higher in children with malaria and iNTS coinfection (24\%) compared to those with malaria alone (10\%). The immunological basis of concurrent malaria and iNTS is poorly understood. We questioned whether or not malaria parasites in children impair phagocytes immunity and induce anti-inflammatory immune response (regulatory CD4 $\mathrm{T}$ cells) and these promote NTS replication and systemic dissemination.

\section{Methods}

We prospectively examined blood killing activity, phagocyte and anti-inflammatory immune responses to NTS in mild malaria infected children ( $\mathrm{n}=50$, aged $6-60$ months) and age matched febrile non-malaria controls $(n=50)$ at Zingwangwa Health Centre. Cases were followed up at day 14 and 30 (convalescence).

\section{Results}

Neutrophil respiratory burst activity was significantly lower during malaria at acute stage (Median 45\%, IQR [25-75]) compared to 1 month into convalescence (Median 89\%, IQR [72.5-94]) and controls (Median 92\%, IQR [85-94]). Blood killing activity to NTS was abrogated when serum was removed, in whole blood condition (Median -0.05 IQR [-0.8-1.0]) compared to washed cells without serum (Median 4.4, IQR [3.5-5.5]). Blood killing activity to NTS increased with age during malaria (acute stage spearman $\mathrm{r}=-0.33,95 \%$ CI $[-0.6,-0.0008]$ and 1 -month spearman $\mathrm{r}=-0.46$, $95 \%$ CI $[-0.7,-0.07)$. CD $4+T$ regs were higher but not statistically significant during acute malaria (Median 12\%, IQR [8.3-18]) compared to day 30 (Median 10\%, IQR [8.6-15]) and controls (Median 9.1\%, IQR [6.9-11.7]).

\section{Conclusions}

In malaria infected children neutrophil immunity is impaired and exhibit higher levels of anti-inflammatory response mediated by CD4+ T regs. Blood killing activity to NTS is age and serum dependent, suggesting antibodies may have an important role.

\section{A7 Modelling climatic and non-climate drivers of malaria for transmission reduction}

James Chirombo ${ }^{1,2}$, Pietro Ceccato ${ }^{3}$, Rachel Lowe ${ }^{4}$, Dianne J. Terlouw ${ }^{2,5}$, Madeleine C. Thomson ${ }^{3}$, Damson Kathyola ${ }^{6}$, Peter J. Diggle ${ }^{1}$, Jonathan M. Read ${ }^{1}$

1. CHICAS, Lancaster Medical School, Lancaster University, United Kingdom

2. Malawi-Liverpool-Wellcome Trust Clinical Research Programme, Blantyre, Malawi
3. International Research Institute for Climate and Society, New York, New York, USA

4. Catalan Institute of Climatic Sciences, Barcelona, Spain

5. Liverpool School of Tropical Medicine, Liverpool, United Kingdmo 6. Ministry of Health, Lilongwe, Malawi

\section{Introduction}

Malaria incidence reduction is a core focus of national and global malaria control efforts. Transmission hotspots identification, through profiling of geographical variation in malaria over time, could inform more targeted intervention strategies. Spatio-temporal statistical models and Bayesian predictive inference are particularly well suited to mapping health outcomes due to their ability to capture spatial variation at large and small scales. We applied these models to investigate the contribution of climatic, environmental and socio-economic factors to district-level variation in malaria incidence in Malawi.

\section{Methods}

We compiled malaria data from an age stratified health management information system database covering all 28 districts in Malawi between July 2004 and December 2015 while socio-economic data were obtained from national household surveys. We combined this with remotely sensed climate and environmental data averaged over the districts to investigate their impact on malaria transmission. Covariates were assessed in a non-spatial generalized linear model to identify significant drivers of malaria, to build a Bayesian hierarchical model and generate predictive risk maps to reveal spatial variation in disease risk. Malaria exceedance probability maps were used to show probabilities of incidence exceeding predefined thresholds.

\section{Results}

We will present the generated predictive risk maps to reveal spatial variation in disease risk to identify areas of unusually high and low risk that could inform sub-district surveillance and control strategies.

\section{Conclusions}

This climate based model approach could be developed into a key component of an integrated national surveillance system, and may be particularly valuable for resource constrained settings like Malawi.

\section{A8 A prospective cohort study evaluating the cardiac safety of dihydroartemisinin- piperaquine for treatment of uncomplicated falciparum malaria in Malawian children}

\section{A. Chipasula-Teleka, E.M. Hodel, D.J. Terlouw}

Malaria Epidemiology Group, Malawi-Liverpool-Wellcome Trust Clinical Research Programme, Blantyre, Malawi

\section{Introduction}

Higher doses of dihydroartemisinin-pipearquine (DHA-PPQ) have been recommended by WHO for treatment of uncomplicated falciparum malaria in children $(<25 \mathrm{~kg})$ in order to achieve adequate exposure needed for efficacy. A known side effect of PPQ is prolongation of QT interval.

The aim was to evaluate the cardiac safety of DHA-PPQ with a normal dose $(<26 \mathrm{mg} / \mathrm{kg})$ and high dose PPQ $(\geq 26 \mathrm{mg} / \mathrm{kg})$ in Malawian children.

\section{Methods}

A total of 100 children treated for uncomplicated malaria at Chikwawa District Hospital, Malawi in ADJuST trial were analyzed. We divided them into 2 groups; DHA-PPQ with normal dose PPQ (74) and high dose PPQ (26). We evaluated the dose/concentration 
relationship with the QT interval and heart rate measured before, during and after the treatment. QT interval was corrected by Bazett's formula.

\section{Results}

DHA-PPQ was well tolerated and did not cause cardiotoxicity among patients in both groups. There were no significant differences in the mean $\triangle \mathrm{QTcB}$ from baseline to treatment completion between the 2 groups; $42 \mathrm{~ms}$ and $43 \mathrm{~ms}$ for normal and high dose PPQ respectively. There was no patient that had a QTcB $\geq 500 \mathrm{~ms}$ during the study. Twenty five and $16 \%$ of normal and high dose group, respectively, had $\Delta \mathrm{QTcB} \geq 60 \mathrm{~ms}$ which resolved by day 28 . Age, height, temperature and concentration had a significant effect on $\triangle \mathrm{QTCB}$.

\section{Conclusions}

DHA-PPQ with normal and high dose PPQ for treatment of uncomplicated falciparum malaria was safe for the heart and did not cause any clinically relevant prolonged QTcB or cardiotoxicity.

\section{A9 Increasing insecticide resistance in Anopheles funestus sensu stricto and An. gambiae sensu lato (Diptera: Culicidae) in Malawi, 2011 to 2015}

\author{
Themba Mzilahowa ${ }^{1}$, Martin Chiumia ${ }^{1}$, Rex B. Mbewe ${ }^{1}$, \\ Veronica Uzalili ${ }^{1}$, Madalitso Luka ${ }^{1}$, Anna Kutengule ${ }^{1}$, Don P. \\ Mathanga ${ }^{1}$, Doreen $\mathrm{Ali}^{2}$, John Chiphwanya ${ }^{2}$, John Zoya ${ }^{2}$, Shadreck \\ Mulenga ${ }^{2}$, Wilfred Dodoli ${ }^{3}$, Jennifer Bergeson-Lockwood ${ }^{4}$, Peter \\ Troell $^{5}$, Jessica Oyugi ${ }^{6}$, Kim Lindblade ${ }^{6}$, John E. Gimnig \\ 1. Malaria Alert Centre, College of Medicine, Blantyre, Malawi \\ 2. National Malaria Control Programme, Ministry of Health, Lilongwe, \\ Malawi \\ 3. WHO Country Office, Lilongwe, Malawi \\ 4.President's Malaria Initiative, United States Agency for International \\ Development, Lilongwe, Malawi \\ 5.President's Malaria Initiative, Centers for Disease Control and \\ Prevention, Lilongwe, Malawi \\ 6. Division of Parasitic Diseases and Malaria, Centers for Disease \\ Control and Prevention, Atlanta, Georgia, USA
}

\section{Introduction}

Susceptibility of principal Anopheles malaria vectors to common insecticides was monitored over a five year period across Malawi to inform and guide the national malaria control programme (NMCP).

\section{Methods}

Adult blood-fed Anopheles spp. and larvae were collected from multiple sites in sixteen districts across the country between 2011 and 2015. First generation $\left(F_{1}\right)$ progeny aged 2-5 days old were susceptibility tested, using standard WHO procedures, against pyrethroids (permethrin and deltamethrin), carbamates (bendiocarb and propoxur), organophosphates (malathion and pirimiphos-methyl) and an organochlorine (DDT).

\section{Results}

Mortality of An. funestus to deltamethrin, permethrin, bendiocarb and propoxur declined significantly over the 5 year (2011-2015) monitoring period. There was wide variation in susceptibility to DDT but it was not associated with time. In contrast, An. funestus exhibited 100\% mortality to the organophosphates (malathion and pirimiphos-methyl) at all sites tested. There was reduced mortality of An. gambiae s.l. to deltamethrin over time though this was not statistically significant. However, mortality of $A n$. gambiae s.l. exposed to permethrin declined significantly over time. Anopheles gambiae exposed to DDT were more likely to be killed if there was high ITN coverage in the mosquito collection area the previous year. There were no other associations between mosquito mortality in a bioassay and ITN coverage or IRS implementation.
Mortality of An. funestus from 4 sites exposed to deltamethrin alone ranged from $2 \%$ to $31 \%$ and from $41 \%$ to $94 \%$ when pre-exposed to the synergist piperonyl butoxide followed by deltamethrin. For permethrin alone, mortality ranged from 2 to $13 \%$ while mortality ranged from $63 \%$ to $100 \%$ when pre-exposed to $\mathrm{PBO}$.

\section{Conclusions}

Pyrethroid resistance was detected in An. funestus and An. gambiae s.l. populations across Malawi and has worsened over the last five years. New insecticides and control strategies are urgently needed to reduce the burden of malaria in Malawi.

\section{A10 Effectof dailytrimethoprim-sulfamethoxazole prophylaxis on the long term clinical impact of malaria infection among HIV infected adults on successful ART in Blantyre, Malawi}

Felix A. Mkandawire ${ }^{1}$, Randy G. Mungwira ${ }^{1}$, Titus H. Divala ${ }^{1}$, Osward M. Nyirenda ${ }^{1}$, Maxwell Kanjala ${ }^{1}$, Lufina E. Tsirizani ${ }^{1}$, Francis Muwalo ${ }^{1}$, Nicaise Ndembi ${ }^{2}$, Terrie E. Taylor ${ }^{3}$, Jane Mallewa ${ }^{4}$, Joep J. van Oosterhout ${ }^{4,5}$, Matthew B. Laurens ${ }^{6}$, Miriam K. Laufer ${ }^{6}$

1. Blantyre Malaria Project, College of Medicine, University of Malawi, Blantyre, Malawi

2. Institute of Human Virology, Abuja, Nigeria

3. Institute for Global Health, University of Maryland School of

Medicine, Baltimore, Maryland, USA

4. Department of Medicine, College of Medicine, University of Malawi,

Blantyre, Malawi

5. Dignitas International, Zomba, Malawi

6. Division of Malaria Research, Institute for Global Health, University of Maryland School of Medicine, Baltimore, Maryland, USA

\section{Introduction}

Sub-Saharan Africa has $90 \%$ and $70 \%$ of all new cases of malaria and HIV respectively. The risk of malaria infection is higher in HIV infected adults. Malaria infection in HIV positive individuals is associated with increased HIV viral load (VL) and decreased CD4+ T-cells. Daily trimethoprim-sulfamethoxazole (TS) reduces the risk of malaria infection in HIV positive individuals but its long term benefit after successful ART has not been well documented.

\section{Methods}

To determine the impact of TS on malaria infection and disease, we analyzed data from clinically stable, non-pregnant HIV infected adults on non-protease inhibitor ART enrolled in an ongoing randomized controlled trial in Blantyre, an area with low to moderate malaria transmission. Participants with CD4 count $>250$ cells/mm3 and VL $<400$ copies/ml were randomized to continue daily TS, discontinue TS, or discontinue TS and begin chloroquine. During the rainy season, we measured asymptomatic infection by quantitative PCR of dried blood spots. Clinical malaria was diagnosed in participants with symptoms suggestive of malaria and positive microscopy.

\section{Results}

We included a subset of participants who continued on TS prophylaxis $(n=34)$ or stopped prophylaxis $(n=27)$. The two groups were similar in age, gender distribution, CD4 count, hemoglobin level and bed net use. Four participants in the TS discontinuation group developed clinical malaria compared to one from the daily TS group. No episodes of asymptomatic malaria infection were detected by PCR.

\section{Conclusions}

Even in this lower transmission setting, TS prophylaxis was 
associated with protection against clinical malaria disease. The absence of asymptomatic malaria infection is in contrast with the common finding of high rates of low-level asymptomatic parasitemia in Malawi. HIV infected adults may be more likely to develop symptomatic disease, or ART or malaria prophylaxis confer some protection. We are currently undertaking immunological evaluation to determine mechanism of this observed phenomenon.

\section{A11 Anopheles funestus sensu stricto Giles (Diptera: Culicidae) bites late in the morning at two rural villages in northern Malawi and implications for malaria vector control}

Themba Mzilahowa ${ }^{1}$, S. Gowelo ${ }^{1}$, J. Chiphwanya ${ }^{2}$, J.H. Banda ${ }^{1}$, A. Bauleni' ${ }^{1}$ M.M. Mukaka ${ }^{3}$

1. Malaria Alert Centre, College of Medicine, University of Malawi, Blantyre, Malawi

2. National Malaria Control Programme, Ministry of Health, Lilongwe, Malawi

3. Department of Community Health, College of Medicine, University of Malawi, Blantyre, Malawi

\section{Introduction}

Understanding the behavior of Anopheles vector mosquitoes provides critical information to guide deployment of malaria vector control interventions especially insecticide-treated bed nets (ITNs) and indoor residual spraying (IRS). Some recent studies have reported shifts in biting behavior in An. funestus, a major malaria vector in sub-Saharan Africa including Malawi. This study aimed to investigate if this phenomenon is true in malaria vectors in Malawi.

\section{Methods}

Human landing catches (HLCs) were carried out at three randomly selected households twice monthly for six months between January and December 2012 at Dambo and Kanyuka villages in Nkhata Bay and Karonga districts in northern Malawi respectively. Mosquitoes were sampled from 18:00 hours in the evening to 06:00 hours in the morning and all hourly collections were put in well labeled separate cups. Subsequently mosquitoes were counted and identified.

\section{Results}

A total of 4,668 and 2,079 mosquitoes were collected over a six months study period (January - August 2014) in Nkhata Bay and Karonga districts respectively. An. funestus s.l was predominant $(55.9 \% ; \mathrm{n}=2,611)$ in Nkhata Bay and An. gambiae s.l was common in Karonga $(34.0 \% ; \mathrm{n}=706)$.

An. funestus was significantly caught biting inside $(\mathrm{p}=0.002)$ while $A n$. arabiensis, preferred biting outside the house $(\mathrm{p}=0.05)$. Furthermore, $A n$. funestus showed a peak biting late in the morning around 4 am when approximately .70 bites were experienced by individuals and remained high till 6 am while An. arabiensis peak biting was around midnight $(12: 00 \mathrm{am})$ when approximately 7 bites/ person were experienced and sharply dropped to 50 bites/ person by the time collections stopped in the morning.

\section{Conclusions}

An. funestus was biting people in the morning when they were outside the protection of ITNs. Further, the tendency by $A n$. arabiensis to bite outdoors would be an important factor in residual malaria transmission.
A12 Comparison of three genotyping methods for resolving the genetic diversity of Plasmodium falciparum field isolates

Tamika J. Sisya ${ }^{1}$, Rachel L. Banda ${ }^{1}$, Standwell C. Nkhoma ${ }^{1,2}$

1. Malawi-Liverpool-Wellcome Trust Clinical Research Programme, Blantyre, Malawi

2. Liverpool School of Tropical Medicine, Liverpool, United Kingdom

\section{Introduction}

With spreading artemisinin resistance and global efforts towards malaria transmission reduction, it is important to correctly diagnose malaria treatment failures from re-infections. We compared two new methods of genotyping with the standard method of genotyping of Plasmodium falciparum merozoite surface proteins 1 and 2 (msp-1 and msp-2).

\section{Methods}

We genotyped Plasmodium falciparum infections $(\mathrm{n}=65)$ to determine the DNA fingerprint of malaria parasites sampled from a single location in Malawi using the classic msp-1 and msp-2 genotyping method, 24 genome-wide single nucleotide polymorphisms (SNPs) and microsatellite genotyping to determine and compare the complexity of each infection.

\section{Results}

Our results indicate that there is a higher concordance $(94 \%)$ between 24 SNP barcoding and microsatellite genotyping, compared with the concordance between msp and microsatellite $(68 \%)$ or msp and 24 SNP barcoding results (65\%). We will present the factors underlying the differences in concordance between these methods, linked to the identification of multiple infections, the complexity of infection, linkage disequilibrium and genotypic richness.

\section{Conclusions}

Our data suggest that msp genotyping underestimates the genetic diversity of infections possibly because it uses fewer genetic markers as compared to 24 SNP barcoding and microsatellite genotyping. Barcoding and microsatellite genotyping methods perform better than msp genotyping to distinguish between recrudescences and reinfections.

\section{HIV/AIDS}

\section{B1 Ready-to-use supplementary food (RUSF) at ART initiation improves nutritional status but does not reduce mortality in severely immunosuppressed HIV-infected adults/older children in sub-Saharan Africa: REALITY trial}

J. Mallewa ${ }^{1}$, P. Mugyenyi ${ }^{2}$, J. Berkley ${ }^{3}$, A.J. Szubert ${ }^{4}$, E. Chidviza ${ }^{5}$, M.J. Thomason ${ }^{4}$, P. Chepkorir ${ }^{6}$, G. Abongomera ${ }^{2}$, K. Baleeta ${ }^{7}$, A. Etyang ${ }^{3}$, C. Warambwa ${ }^{5}$, B. Melly ${ }^{6}$, S. Mudzingwa ${ }^{5}$, C. Kelly ${ }^{1}$, C. Agutu ${ }^{3}$, H. Wilkes ${ }^{4}$, V. Musiime ${ }^{2}$, A. Lugewma ${ }^{8}$, S.L. Pett ${ }^{4}$, M. Bwakura-Dangarembizi ${ }^{5}$, A.J. Prendergast ${ }^{4,9}$, A.S. Walker ${ }^{4}$, D.M. Gibb $^{4}$, REALITY trial team

1. Department of Medicine, College of Medicine, University of Malawi, Blantyre, Malawi

2. Joint Clinical Research Centre, Kampala, Uganda

3. KEMRI-Wellcome Trust Research Programme, Kilifi, Kenya

4. MRC Clinical Trials Unit at UCL, London, United Kingdom

5. University of Zimbabwe Clinical Research Centre, Harare, Zimbabwe

6. School of Medicine, Moi University, Eldoret, Kenya

7. Joint Clinical Research Centre, Mbale, Uganda

8. Joint Clinical Research Centre, Mbarara, Uganda

9. Queen Mary University of London, London, United Kingdom 


\section{Introduction}

Early mortality after antiretroviral therapy(ART) initiation is high among HIV-infected adults/children with severe immunosuppression in sub-Saharan Africa. Baseline malnutrition is common and increases mortality, but nutritional supplementation is generally only provided to those with severe malnutrition. Whether universal provision in advanced disease would improve nutritional status and reduce early mortality is unknown.

\section{Methods}

REALITY 2x2x2-factorial open-label randomised trial (ISRCTN43622374) enrolled ART-naïve HIV-infected adults/ children >5years with CD $4<100$ cells $/ \mathrm{mm}^{3}$ from Kenya, Malawi, Uganda, Zimbabwe. Randomisation compared initiating ART with/without 12 weeks of Ready-to-Use Supplementary Food(RUSF), providing $1000 \mathrm{kcal} /$ day with multi-vitamins/ minerals. Those with severe malnutrition received Ready-to-Use Therapeutic Food(RUTF) regardless of randomisation. Primary endpoint was 24-week mortality.

\section{Results}

1805 eligible adults $(\mathrm{n}=1733 ; 96.0 \%)$ and children/adolescents $(n=72 ; 4.0 \%)$ were enrolled,median age 36years; $53.2 \%$ male; $53.7 \%$ WHO stage $3 / 4$, and median baseline CD4 36cells $/ \mathrm{mm}^{3}$ (IQR 16-62). For those $\geq 13$ years, median baseline weight was $53 \mathrm{~kg}$ (IQR 47-60), BMI 19.3kg/ $\mathrm{m}^{2}$ (17.4-21.5) and MUAC 24.0 $\mathrm{cm}(22.0-$ 26.1). Participants were randomised to RUSF ( $\mathrm{n}=897$ ) or no-RUSF $(\mathrm{n}=908)$ with ART. $19(2.1 \%)$ and 32(3.5\%) respectively received RUTF, following local guidelines. Follow-up was 48weeks $(3.8 \%$ loss-to-follow-up). Gains in weight, BMI and MUAC were greater in the RUSF group ( $p=0.004,0.005,0.03)$. Maximum differences were at 12 weeks; +3.8 RUSF versus $+2.9 \mathrm{~kg}$ no-RUSF, +1.4 versus $+1.1 \mathrm{~kg} / \mathrm{m}^{2}$, and +1.2 versus $+1.0 \mathrm{~cm}$ respectively. There were no differences in grip strength $(\mathrm{p}=0.35) .96(10.9 \%)$ RUSF versus $92(10.3 \%)$ no-RUSF died before 24 weeks (adjusted hazard ratio $=1.07(95 \%$ CI $0.80-1.42 \mathrm{p}=0.65)$ to 48 -weeks $(\mathrm{p}=0.86)$.

\section{Conclusions}

RUSF supplementation at ART initiation in advanced disease improved nutritional status but did not impact mortality.

\section{B2 The influence of masculinity on HIVST community intervention: A qualitative evaluation of empirical evidence from Blantyre, Malawi}

\author{
Moses Kumwenda ${ }^{1,2}$, A. Munthali ${ }^{3}$, A. Choko ${ }^{1,4}$, J. Chikovore $^{5}$, \\ M. Nliwasa ${ }^{4}$, R. Sambakunsi ${ }^{1}$, G. Chipungu ${ }^{2}$, M. Mwapasa ${ }^{2}$, K. \\ Kaswaswa $^{2}$, T. Gutteberg ${ }^{6}$, E.L. Corbett ${ }^{4}$, N. Desmond ${ }^{7}$ \\ 1.Malawi-Liverpool-Wellcome Trust Clinical Research Programme, \\ Blantyre, Malawi \\ 2. College of Medicine, University of Malawi, Blantyre, Malawi \\ 3. Centre for Social Research, Chancellor College, University of Malawi, \\ Zomba, Malawi \\ 4. London School of Hygiene and Tropical Medicine, London, United \\ Kingdom \\ 5. Human Sciences Research Council, Pretoria, South Africa \\ 6. University of Tromso, Tromso, Norway \\ 7. University of Liverpool, Liverpool, United Kingdom
}

\section{Introduction}

For HIV, population surveys and cohort outcomes show men to have a higher risk of undiagnosed disease than women, and higher risk of death following diagnosis. Here we report findings from an HIV self-testing (HIVST) study with relevance to other chronic conditions, including TB. HIVST addresses barriers associated with traditional models of providing HIV testing, giving notably high male participation. We examined the role of masculinity on HIVST decisions and subsequent actions amongst couples in urban Blantyre.

\section{Methods}

Sixty seven self-tested participants were interviewed at baseline, 49 at first follow-up (3-5 months) and 50 at second follow-up (12-15 months). Content analysis was used to interpret study findings.

\section{Results}

Conception of masculinity dictated men's actions and prescribed specific behavioral attributes, namely having control, knowledge, strength and toughness, and both sexual and economic productivity. The mandate of constant economic provision prevented men living a hand-to-mouth existence from testing with their partners, as they were not at home when HIVST was offered. Notions of men as all-knowing could promote denial of positive HIVST results, with men allowed to question their authenticity especially when their partner had tested negative. Even when positive HIVST results were believed, notions of strength and resilience, combined with the relentless demands of their household provider role, dissuaded men from promptly seeking HIV care, as they felt no urgency to act when healthy. Notions of sexual productivity encouraged men to insist unprotected sex irrespective of their own or their partner's HIV status.

\section{Conclusions}

The social construction of masculinity, which includes relentless pressure to earn in this impoverished urban setting, restricted the potential benefits of early HIV care and prevention, even within established couples. Community HIV strategies need to explicitly account for the obstacles and barriers linked to masculinity to have full impact.

\section{B3 Maternal characteristics, PMTCT programme utilization and confirmation of HIV status in a nationally representative sample of women screened at 4-26 weeks postpartum in Malawi}

M. van Lettow ${ }^{3,4}$, M. Landes ${ }^{3,4}$, J.J. van Oosterhout ${ }^{3.5}$, E. Schouten ${ }^{2}$, H. Phiri ${ }^{2}$, E. Nkhoma ${ }^{2}$, T. Kalua ${ }^{6}$, S. Gupta ${ }^{1}$, B. Tippett-Barr' ${ }^{1}$; on behalf of the NEMAPP Consortium

1. Centers for Disease Control and Prevention, Lilongwe, Malawi 2. Management Sciences for Health (MSH) Malawi

3. Dignitas International, Zomba, Malawi

4. University of Toronto, Canada

5. College of Medicine, University of Malawi, Blantyre, Malawi 6. Ministry of Health, Lilongwe, Malawi

\section{Background}

The National Evaluation of the Malawi PMTCT Program aims to evaluate the effectiveness of the integrated approach towards elimination of MTCT of HIV in Malawi. To identify a representative sample of HIV-exposed infants for inclusion in a prospective evaluation, mother-infant-pairs were screened for HIV at 4-26 weeks postpartum. This paper describes maternal characteristics, PMTC program utilization and ELISA confirmed maternal HIV status, by known HIV status during pregnancy.

\section{Methods}

Mothers were consented and screened while attending an under-5 clinic with their infant in 54 randomly selected health facilities across 10 districts. Mothers were interviewed and HIV rapidtesting was conducted at site, followed by batched confirmatory ELISA testing in a reference laboratory.

\section{Results}

A total of 30,822 mothers were screened (Nov'14- Mar'16). HIV status was known/ascertained during last pregnancy in $>95 \%$ of mothers. Median age and parity among HIV-uninfected and infected mothers was 23(IQR20-29) and 29(24-34) years, and 2(IQR1-3) and 3(IQR2-5), respectively. Among 2961(9.6\%) known HIV- 
infected mothers, $45.9 \%$ started ART during the last pregnancy; $48.7 \%$ prior to that; $1.5 \%$ post-partum, and $3.8 \%$ did not start. HIV testing confirmed $30008 / 30411(98.7 \%)$ of the HIV status ascertained during pregnancy. An additional 290 infected mothers were identified; 56/471(11.9\%) among women with unknown status and 234/27495(0.9\%) with HIV-negative status during last pregnancy. A total of $175(0.6 \%)$ test results were discordant; of which $26(0.9 \%)$ were false previous positives, $11(0.04 \%)$ presumed false positive rapid-tests, and $138(0.4 \%)$ false negatives rapid tests.

\section{Conclusion}

Utilization of PMTCT services under option B+ in Malawi is high; $>95 \%$ of women at ANC had their HIV status ascertained or confirmed and almost $50 \%$ of HIV infected women were already on ART prior to their recent pregnancy. A significant number of (presumed) new and possible acute infections were identified 4-26 weeks postpartum, highlighting missed opportunities for prevention of MTCT in a high risk group.

\section{B4 12-week raltegravir-intensified quadruple-} therapy versus triple first-line ART reduces viral load more rapidly but not mortality in severely immunosuppressed African HIVinfected adults/older children: The REALITY trial

$\underline{\text { C. Kityo }}^{1}$, A. Siika ${ }^{2}$, A.J. Szubert ${ }^{3}$, J. Mallewa ${ }^{4}$, M. BwakuraDangarembizi $^{5}$, S. Kabahenda ${ }^{6}$, S. Mwaringa ${ }^{7}$, S.L. Pett ${ }^{3}$, A. Griffiths $^{3}$, A. Lugemwa ${ }^{8}$, S. Wachira ${ }^{2}$, G. Musoro ${ }^{5}$, C. Rajapakse ${ }^{3}$, T. Etyang ${ }^{7}$, J. Abach ${ }^{9}$, P. Wavamunno ${ }^{1}$, L. Nyondo-Mipando ${ }^{4}$, A. $\operatorname{Reid}^{5}$, K. Nathoo ${ }^{5}$, J. Hakim 5 , D.M. Gibb ${ }^{3}$, A.S. Walker ${ }^{3}$, REALITY trial team

1. Joint Clinical Research Centre, Kampala, Uganda,

2. School of Medicine, Moi University, Eldoret, Kenya

3. MRC Clinical Trials Unit at UCL, London, United Kingdom

4. Department of Medicine, College of Medicine, University of Malawi; and Malawi-Liverpool-Wellcome Trust Clinical Research Programme,

Blantyre, Malawi,

5. University of Zimbabwe Clinical Research Centre, Harare, Zimbabwe

6. Joint Clinical Research Centre, Fort Portal, Uganda

7. KEMRI-Wellcome Trust Research Programme, Kilifi, Kenya

8. Joint Clinical Research Centre, Mbarara, Uganda

9. Joint Clinical Research Centre, Gulu, Uganda

\section{Introduction}

Early mortality after initiating antiretroviral therapy (ART) is high among HIV-infected adults and children with advanced disease in Sub-Saharan Africa. Intensifying ART with an integrase inhibitor should reduce viral load (VL) faster, whether this reduces early mortality is unknown.

\section{Methods}

The REALITY 2x2x2-factorial open-label trial (ISRCTN43622374) randomised ART-naïve. HIV-infected adults and children $>5$ years with CD $4<100$ cells $/ \mathrm{mm}^{3}$ from Kenya, Malawi, Uganda, Zimbabwe. Randomisation compared initiating ART with 2NRTI+NNRTI with/without 12-week raltegravir intensification.

\section{Results}

1805 eligible adults $(\mathrm{n}=1733 ; 96.0 \%)$ and children/adolescents $(\mathrm{n}=72 ; 4.0 \%)$, (median 36years; $53.2 \%$ male) were randomised to raltegravir-intensified ( $\mathrm{n}=903)$ or standard-ART $(\mathrm{n}=902)$ and followed for 48 weeks (3.8\% loss-to-follow-up). Median baseline CD4 was 36cells/mm3 (IQR 16-62) and VL 230,000c/ $\mathrm{ml}(72.5 \% \geq 100,000 \mathrm{c} / \mathrm{ml})$. At $4,12,24$ and 48 weeks, VL was $<50 \mathrm{c} / \mathrm{ml}$ in $42.8 \%, 74.1 \%, 77.2 \%$ and $82.9 \%$ in raltegravirintensified versus $14.5 \%, 54.6 \%, 76.0 \%$ and $79.5 \%$ standard-ART ( $\mathrm{p}<0.001,<0.001,0.59,0.12$, respectively). CD4 increases through
24 weeks were similar $(\mathrm{p}=0.82)$, although a small difference became apparent at 48 weeks $(+163$ cells $/ \mathrm{mm} 3$ intensified versus +148 cells $/ \mathrm{mm} 3$ standard, $\mathrm{p}=0.04) .97(10.9 \%)$ intensified versus 91(10.2\%) standard-ART died before 24 weeks (adjusted hazardratio $[\mathrm{aHR}]=1.09(95 \%$ CI $0.82-1.46) \mathrm{p}=0.54) ; 110(12.4 \%)$ versus $115(13.0 \%)$ respectively died before 48 weeks $(\mathrm{aHR}=0.98(0.75$ 1.27) $\mathrm{p}=0.86$ ). There was no difference in time to first WHO $3 / 4$ event or death $(p=0.31)$. Serious adverse events (AEs), grade $3 / 4$ AEs and drug-related AEs (adjudicated blind to randomisation) were similar $(\mathrm{p}>0.3)$.

\section{Conclusions}

12-week raltegravir-intensified ART was well tolerated, resulted in faster VL reduction through 24 weeks and increased CD4 at 48 weeks, but did not reduce mortality or WHO 3/4 events.

\section{B5 Adherence to antiretroviral therapy during and after pregnancy: Cohort study on women receiving care in Malawi's "Option B+" programme}

Andreas D. Haas ${ }^{1}$, Malango T. Msukwa ${ }^{1,2}$, Matthias Egger ${ }^{1,7}$, Lyson Tenthani ${ }^{1,3}$, Hannock Tweya ${ }^{4,3,1}$, Andreas Jahn ${ }^{6,3}$, Oliver J. Gadabu $^{3}$, Kali Tal ${ }^{1}$, Luisa Salazar-Vizcaya ${ }^{1}$, Janne Estill ${ }^{1}$, Adrian Spoerri ${ }^{1}$, Nozgechi Phiri ${ }^{1}$, Frank Chimbwandira ${ }^{5}$,Joep J. van Oosterhout ${ }^{8,9}$, Olivia Keiser ${ }^{1}$

1. Institute of Social \& Preventive Medicine, University of Bern, Bern, Switzerland

2. The Baobab Health Trust, Lilongwe, Malawi

3. International Training \& Education Center for Health Malawi, Lilongwe, Malawi

4. The International Union Against Tuberculosis and Lung Disease, Paris, France

5. Lighthouse Trust, Lilongwe, Malawi

6. Department of HIV and AIDS, Ministry of Health, Lilongwe, Malawi 7. Centre for Infectious Disease Epidemiology and Research, University of Cape Town, Cape Town, South Africa

8. Dignitas International, Zomba, Malawi

9. Department of Medicine, College of Medicine, University of Malawi, Blantyre, Malawi

\section{Introduction}

Adherence to antiretroviral therapy (ART) is crucial to preventing mother-to-child transmission of HIV and ensuring the long-term effectiveness of ART, yet there are few data from African routine care programmes on maternal adherence to triple ART.

\section{Methods}

We analyzed data of women who started ART at 13 large health facilities in Malawi between September 2011 and October 2013. We defined adherence as the percentage of days "covered" according to pharmacy claims. Adherence of $\geq 90 \%$ was deemed adequate. We calculated inverse probability of censoring weights to adjust adherence estimates for informative censoring. We used descriptive statistics, survival analysis and pooled logistic regression to compare adherence between three groups: pregnant and breastfeeding women eligible for ART under Option B + , and non-pregnant and non-breastfeeding women who started ART with low CD4 and/or WHO clinical stage 3/4 disease.

\section{Results}

Adherence was adequate for $73 \%$ of the women during pregnancy, for $66 \%$ in the first three months postpartum, and for about $75 \%$ during months 4-21 postpartum. About $70 \%$ of women who started ART during pregnancy and breastfeeding adequately adhered during the first 2 years of ART, but only about $30 \%$ of them maintained adequate adherence at every visit. Risk factors for inadequate adherence included starting ART with an Option $\mathrm{B}+$ indication, at a younger age, or at a district hospital or health centre. 


\section{Conclusions}

One-third of women retained in the Option $\mathrm{B}+$ programme inadequately adhered during pregnancy and breastfeeding, especially soon after delivery. Effective interventions to improve adherence among Option $\mathrm{B}+$ women should be implemented.

B6 The effect of type of progestin-only contraception (DMPA versus levonorgestrel implant) on HIV viral shedding in the genital tract of HIV-infected women on antiretroviral therapy

Lameck Chinula ${ }^{1,2,3}$, Jeffrey Wiener ${ }^{4}$, Jennifer Tang ${ }^{1,2,3}$, Julie A.E. Nelson ${ }^{5}$, Stacey Hurst ${ }^{4}$, Gerald Tegha ${ }^{2}$, Albans Msika ${ }^{2}$, Sascha Ellington ${ }^{4}$, Mina Hosseinipour ${ }^{2,6}$, Ronald Mataya ${ }^{3,7}$, Lisa B. Haddad $^{8}$, Athena P. Kourtis ${ }^{4}$

1. Department of Obstetrics \& Gynecology, University of North Carolina at Chapel Hill, Chapel Hill, North Carolina, USA

2. UNC Project-Malawi, Lilongwe, Malawi

3. Department of Obstetrics \& Gynecology, College of Medicine, University of Malawi, Blantyre, Malawi;

4. Centers for Disease Control and Prevention, Division of Reproductive Health, Atlanta, Georgia, USA

5. Department of Microbiology and Immunology, University of North Carolina at Chapel Hill, Chapel Hill, North Carolina, USA

6. Division of Infectious Diseases, University of North Carolina at

Chapel Hill, Chapel Hill, North Carolina, USA

7. School of Public Health, Loma Linda University, Loma Linda,

California, USA

8. Department of Obstetrics \& Gynecology, Emory University, Atlanta, Georgia, USA

\section{Introduction}

Hormonal contraception, particularly injectable contraception, has been linked in some studies to higher genital HIV shedding and risk of HIV transmission to partners. There is very limited information about this association in the context of antiretroviral therapy (ART). We assessed the effect and compared the impact of two progestin-containing contraceptives, the Depot Medroxyprogesterone Acetate (DMPA) injectable and the 5-year Levonorgestrel (LNG) implant, on HIV shedding in the genital tract of HIV-infected women.

\section{Methods}

This analysis is from a randomized trial evaluating the effects of DMPA and LNG-implant on the genital tract of HIV-infected and uninfected women in Malawi. HIV-1 RNA viral load (VL) was measured in cervicovaginal lavage using the Abbott RealTime assay. We compared the frequency and magnitude of genital shedding at visits before and for 6 months after initiation of contraception and between arms using repeated measurement models fit by generalized estimating equations.

\section{Results}

We randomized 73 HIV-infected women: 37 to DMPA and 36 to LNG-implant. Eighty-nine percent of DMPA users and 97\% of LNG-implant users were on ART at enrollment. Among women on ART, $6.1 \%$ of DMPA users and $5.7 \%$ of LNG-implant users had detectable genital HIV at enrolment. The frequency of genital shedding and the genital VL were not different before and after contraceptive initiation $[\mathrm{RR}=1.01(95 \% \mathrm{CI}=0.98-1.04)]$ or between the two arms $[\mathrm{RR}=1.01(95 \% \mathrm{CI}=0.98-1.04)]$ among women on ART at enrollment. Genital shedding was uncommon $(<10 \%$ at any time point) and of low magnitude (maximum 3,478 copies/ml).
B7 Early HIV transmission in 4- to 12-week-olds in Malawi's PMTCT Option B+ programme

Beth A. Tippett Barr ${ }^{1}$, Erik Schouten ${ }^{1}$, Joep J. van Oosterhout ${ }^{1}$, Sundeep Gupta ${ }^{1}$, Happy Phiri ${ }^{2}$, Frank Chimbwandira, Deus Thindwa ${ }^{2}$, Christopher Blair ${ }^{2}$, Andreas Jahn ${ }^{5}$, Monique van Lettow $^{3}$

1. Centers for Disease Control and Prevention, Lilongwe, Malawi

2. Management Sciences for Health (MSH)

3. Dignitas International, Zomba, Malawi

4. Department of Medicine, College of Medicine, University of Malawi, Blantyre, Malawi

5. Department of HIV and AIDS, Ministry of Health, Lilongwe, Malawi 6. I-TECH Malawi, Lilongwe, Malawi

\section{Background}

Option B+ was conceptualized and implemented in Malawi in 2011, and a two year cohort study called the National Evaluation of Malawi's PMTCT Program (NEMAPP) was implemented in November 2014. A primary objective of NEMAPP is to measure national mother-to-child-transmission (MTCT) in the era of Option $\mathrm{B}+$, and determine Malawi's progress towards the virtual elimination of MTCT.

\section{Methods}

NEMAPP was implemented at 54 health facilities in 10 districts. A stratified cluster sampling design was used to identify a nationally representative sample of 4-12 week old infants. Mothers were consecutively consented and screened for HIV while attending an under-5 clinic, and all identified HIV-exposed infants underwent HIV-1 DNA testing. This paper presents results of early infant transmission at the time of enrolment into NEMAPP. Complex weighted survey design analysis was conducted using SPSS.

\section{Results}

Amongst 2,125 HIV-positive mothers of 4-12 week old infants, 2,082 $(96.1 \%)$ reported knowing their HIV status in pregnancy, and $1,865(88.5 \%)$ were on ART in pregnancy. Overall MTCT was $4.2 \%$ (95\% CI 2.9-6.1); for women on ART in pregnancy, MTCT was $2.5 \%$ (95\% CI 1.6-3.9). Of the women not on ART in pregnancy, MTCT was $17.9 \%$ (95\% CI 13.0 24.2). MTCT varied by timing of ART initiation: from $1.4 \%(95 \%$ CI $0.5-3.9)$ in those on ART before pregnancy, to $20.2 \%$ (95\% CI 5.8-50.7) in those starting ART post-partum.

\section{Conclusions}

Five years after trailblazing the way globally with Option $\mathrm{B}+$, Malawi's MTCT rates under Option B+ are comparable to that of developed nations. The largest proportional contribution to new pediatric infections is from the small percentage of HIV-positive women not on ART in pregnancy. With the current sustained emphasis on routine testing in ANC to close the remaining treatment gap, Malawi will be ready to submit a validation request for virtual elimination of MTCT to WHO before 2020.

\section{B8 Enhanced infection prophylaxis reduces mortality in severely immunosuppressed HIV- infected adults and older children initiating antiretroviral therapy in Kenya, Malawi, Uganda and Zimbabwe: the REALITY trial}

J. Hakim ${ }^{1}$, V. Musiime 2 , A.J. Szubert ${ }^{3}$, A. Siika ${ }^{4}$, J. Mallewa ${ }^{5}$, C. Agutu $^{6}$, S.L. Pett ${ }^{3}$, M. Bwakura- Dangarembizi ${ }^{1}$, A. Lugemwa ${ }^{7}$, S. Kaunda ${ }^{5}$, M. Karoney ${ }^{4}$, K. Maitland ${ }^{6}$, A. Griffiths ${ }^{3}$, C. Kityo ${ }^{2}$, P. Mugyenyi ${ }^{2}$, A.J. Prendergast ${ }^{3,8}$, A.S. Walker ${ }^{3}$, D.M. Gibb ${ }^{3}$, REALITY trial team 
3. MRC Clinical Trials Unit at UCL, London, United Kingdom

4. School of Medicine, Moi University, Eldoret, Kenya

5. Department of Medicine, College of Medicine, University of Malawi; and Malawi-Liverpool-Wellcome Trust Clinical Research Programme,

Blantyre, Malawi

6. KEMRI-Wellcome Trust Research Programme, Kilifi, Kenya

7. Joint Clinical Research Centre, Mbarara, Uganda

8. Queen Mary University of London, London, United Kingdom

\section{Introduction}

Mortality from infections is high in the first 6 months of antiretroviral therapy (ART) among HIV-infected adults and children with advanced disease in sub-Saharan Africa. Whether an enhanced package of infection prophylaxis at ART initiation would reduce mortality is unknown.

\section{Methods}

The REALITY 2x2x2factorial open-label trial (ISRCTN43622374) randomised ART-naïve HIV-infected adults and children $>5$ years with CD $4<100$ cells $/ \mathrm{mm}^{3}$. Randomisation compared initiating ART with enhanced prophylaxis (continuous cotrimoxazole plus 12 weeks isoniazid/pyridoxine (anti-tuberculosis); fluconazole (anti-cryptococcal/candida); 5 days azithromycin (anti-bacterial/ protozoal); and single-dose albendazole (anti-helminth), versus standard-of-care cotrimoxazole. The primary endpoint was 24 week mortality.

\section{Results}

1805 eligible adults $(\mathrm{n}=1733 ; 96.0 \%)$ and children/adolescents $(\mathrm{n}=72 ; 4.0 \%$ ) (median 36 years; $53.2 \%$ male) were randomised to enhanced $(n=906)$ or standard prophylaxis $(n=899)$ and followed for 48 weeks (3.8\% loss-to-follow-up). Median baseline CD4 was 36 cells $/ \mathrm{mm}^{3}$ (IQR 16-62), $47.3 \%$ were WHO stage $1 / 2$. $80(8.9 \%)$ enhanced versus $108(12.2 \%)$ standard prophylaxis died before 24 weeks (adjusted hazard ratio [aHR] $=0.73$; 95\% CI $0.54-0.97 ; \mathrm{p}=0.03)$ and $98(11.0 \%)$ versus $127(14.4 \%)$, respectively, died before 48 weeks $(\mathrm{aHR}=0.75 ; 95 \%$ CI $0.58-0.98$; $\mathrm{p}=0.04)$. Enhanced prophylaxis significantly reduced incidence of tuberculosis $(p=0.02)$, cryptococcal disease $(p=0.01)$, oral/ oesophageal candidiasis $(\mathrm{p}=0.02)$, deaths of unknown cause $(p=0.02)$, and hospitalisations $(p=0.06)$ but not presumed severe bacterial infections $(p=0.38)$. Serious and grade 4 adverse events were marginally less common with enhanced prophylaxis $(\mathrm{p}=0.06)$. CD4 increases and VL suppression were similar between groups $(\mathrm{p}>0.2)$.

\section{Conclusions}

Enhanced infection prophylaxis at ART initiation reduces early mortality by $25 \%$ among HIV-infected adults and children with advanced disease. The pill burden did not adversely affect VL suppression. Policy-makers should consider adopting and implementing this low-cost broad infection prevention package which could save 3.3 lives/100 individuals treated.

\section{B9 Modelling the effect of vaccination and quarantine on tuberculosis dynamics}

\section{John D. Mutepuwa, L.K. Eneya, M. Maliyoni}

Chancellor College, University of Malawi, Zomba, Malawi

\section{Introduction}

Tuberculosis is caused by a bacteria called Mycobacterium tuberculosis (MTB). One- third of the global population is affected with MTB and 7 to 8 million cases occur every year. In Africa 1.5 million new TB cases occur each year. Tuberculosis remains a major public health problem in Malawi. It is one of the leading causes of morbidity and mortality as such a Mathematical study assess the effect of vaccination and quarantine on tuberculosis dynamics.

\section{Methods}

A mathematical model using an SEIR framework for the dynamics of tuberculosis formulated incorporating vaccination and quarantine and analyzed in order to establish the effect of vaccination and quarantine on tuberculosis dynamics. The equilibrium points of the model are determined qualitatively.

\section{Results}

The disease-free equilibrium point is locally asymptotically stable whenever the effective reproduction ratio is less than one and the endemic equilibrium point is locally asymptotically stable whenever the effective reproduction ration is greater than one (analytical results). Tuberculosis epidemic can be reduced when vaccination is introduced to individuals and also quarantine is introduced to infected individuals (numerical results).

\section{Conclusions}

Vaccination and quarantine are the important disease preventive and control measures respectively as it reduces the number of infected individuals and further reduces the spread of the disease. Thus, if more people are vaccinated and more infectious people are put on quarantine then TB can be reduced.

\section{B10 Evaluating the accuracy of a low-cost diagnostic test for susceptible and drug- resistant tuberculosis}

$\underline{\text { E. Jumbe }}^{1}$, A. Zhang ${ }^{2}$, E. Chemey ${ }^{1}$, C. Evans $^{3}$, C. Kamba ${ }^{4}$, A. Norris ${ }^{5}$, X. Pan' ${ }^{2}$, V. Mwapasa ${ }^{6}$, S. Wang ${ }^{2}$, J.B. Torrelles ${ }^{2 *}$, J. Kwiek ${ }^{2 *}$

1. Child Legacy International, Umoyo wa Thanzi Research, Msundwe, Malawi

2. College of Medicine, The Ohio State University, Columbus, Ohio, USA 3. Imperial College London, London, United Kingdom

4. Ministry of Health, Lilongwe, Malawi

5. College of Public Health, The Ohio State University, Columbus, Ohio, USA

6. College of Medicine, University of Malawi, Blantyre, Malawi

\section{Introduction}

While acid-fast bacilli (AFB) smear is the most common tuberculosis (TB) diagnostic method in Malawi, it is labor intensive, has lessthan-ideal sensitivity, and cannot assess TB drug susceptibility. TB color plates (called CX-test), which simultaneously diagnoses TB and TB drug-susceptibility in 14 days, are a low cost, simple, highly sensitive alternative TB diagnostic. TB CX-test has been tested extensively in research laboratories, but its performance in realworld settings is not well characterized.

\section{Methods}

In this proof of concept study, to date we enrolled 54 suspected TB patients at a rural hospital in Malawi. Participants provided basic demographic data and submitted sputum samples for TB testing using AFB staining and the TB CX-test. Discordant samples were resolved by GeneXpert. We calculated sensitivity, specificity, positive- and negative predictive values for the TB CX-test.

\section{Results}

An interim analysis of the ongoing study shows that 9 of the 54 participants $(17 \%)$ had a positive TB diagnosis. One sample was AFB smear negative but TB-CX and GeneXpert positive; the remaining samples were concordant between tests. The TB CXtest was highly sensitive $(100 \%)$ and specific $(100 \%)$. At a range of TB prevalence values, PVP and NPV were calculated to be $100 \%$. The TB CX-test detected a small number of drug resistant samples $(6 \%)$.

\section{Conclusions}

Our preliminary findings demonstrate that the TB CX-test 
accurately identifies TB and may be an effective, low cost TB diagnostic with the potential to simultaneously diagnose TB and test for drug sensitivity.

\section{B11 Delayed submission of sputum specimen by adult tuberculosis suspects in Mzimba District, Malawi}

\author{
John L.Z. Nyirenda ${ }^{1}$, K. Phiri ${ }^{2}$ \\ 1. University of Livingstonia, Livingstonia, Malawi \\ 2. College of Medicine, University of Malawi, Blantyre, Malawi
}

\section{Introduction}

Tuberculosis continues to be a major cause of morbidity and mortality in the world although the disease is curable even in the presence of HIV infection. Sputum examination is one of the methods through which TB cases are detected from the community hence timely submission of sputum specimen is vital in Pulmonary TB case detection.

\section{Methods}

The study was a cross-sectional study that applied both quantitative and qualitative approaches.

\section{Results}

The results indicated that $61.5 \%(\mathrm{~N}=410, \mathrm{P}-\mathrm{v}<0.001)$ of the participants had adequate knowledge about transmission of TB. The results also indicated that $20.5 \%$ of the participants were not comfortable to disclose or talk about a relatives TB status and $32.0 \% ; \mathrm{P}-\mathrm{v}<0.000$, of the participants believe that there are traditional drugs that cure cough.

\section{Conclusions}

There is an inadequate knowledge and low levels of awareness of transmission and basic aetiological information of TB. There is inadequate accessibility to service points. There are socialeconomic and cultural beliefs that contribute to delay. There are existing knowledge gaps, beliefs and community TB control operational challenges that affect submission of sputum specimen by adult TB suspects in Mzimba District.

\section{B12 Utility of counselling within HIVST from the perspective of HIV-negative individuals exposed to HIV self-testing}

\author{
Mwiza Sambo ${ }^{1}$, Wezzie Lora ${ }^{1,2}$, Moses Kumwenda ${ }^{1}$, Nicola \\ Desmond $^{1,2}$ \\ 1. Malawi-Liverpool-Wellcome Trust Clinical Research Programme, \\ Blantyre, Malawi \\ 2. Liverpool School of Tropical Medicine, Liverpool, United Kingdom
}

\section{Introduction}

Recent research on community based HIV self-testing in Malawi highlights community preferences for home-based testing and emphasizes the ethical requirement to provide counselling within an HIV self-testing delivery model. We know that the package of HIV Counselling and Testing has effectiveness for HIV discordant couples and HIV positive individuals. However there is no clear evidence on the effectiveness of counselling in HTC context for HIV negative individuals

\section{Methods}

This paper focuses on serial biographical interviews taken at time point 0 and 3 months post HIV with individuals aged 16 to 49 Model used was a semi restricted model in which community distribution agents provided support to testers.

\section{Results}

Both men and women reported to have benefitted from the counselling though women were more positive towards counselling. Counselling offered was convenient, private and autonomous thereby removed stigma associated with HIV test results. However, there were concerns around counselling because it was mainly focused on an individual and not the social networks that clients had. Thus the counselling only helped to manage the immediate and clinical shocks after HIV self-testing and not the broader contextual issues. Complaints were raised on the deficiencies in the content of counselling provided.

\section{Conclusions}

Counselling is considered useful to testers irrespective of the test outcomes. However counselling is not different between models since it targets the individuals and aims to address the immediate post-test reactions. Individual targeted counselling does not account for complexity of behaviour embedded in broader contextual dynamic and longer time period so effect overtime diminishes especially for HIV negative individuals.

\section{HEALTH SYSTEMS AND NONCOMMUNICABLE DISEASES}

\section{C1 Factors affecting the utilization of electronic medical records system at Queen Elizabeth Central Hospital and Kamuzu Central Hospital in Malawi}

\section{$\underline{\text { Khwima Esther Mkalira }}{ }^{1}$, Andrew Kumitawa ${ }^{1}$, Benjamin} Kumwenda

1. School of Public Health and Family Medicine, College of Medicine, University of Malawi, Blantyre, Malawi

2. Faculty of Biomedical Sciences and Health Professions, College of Medicine, University of Malawi, Blantyre, Malawi

\section{Introduction}

Manual records keeping in the Malawian health management information system has been a challenge because of difficulties in accessing patients' records, loosing of health passports by patients, problems in patient tracking and many more. Despite introduction of electronic medical records system (EMR) in 2001, manual records system has been in parallel use. Some health workers still prefer using the manual systems than EMRs. This research addresses two main research questions: What are the factors that affect the utilization of EMRs in Malawian hospitals? To what extent do these factors affect the delivery of health services?

\section{Methods}

This was a cross-sectional study which used both quantitative and qualitative methods. There were 111 randomly selected health workers that were interviewed. The respondents were health workers from Queen Elizabeth and Kamuzu Central Hospitals. Data was collected using a questionnaire and an interview guide. Quantitative data was analyzed using the Stata version 12 while content analysis was used to analyse qualitative data.

\section{Results}

The study found that age, gender and previous computer experience had no significant influence on the utilization of EMR. In contrast, educational levels and staff job title affected EMR use. Computer network problems, lack of EMR training and managerial support negatively affected the utilization of the EMR. 


\section{Conclusions}

These findings provide adequate information that could guide hospital management, system designers, implementers and users on measures of improving the use of the EMRs to improve health service delivery.

\section{C2 Early experiences of integrating noncommunicable disease screening and treatment in a large ART clinic in Zomba, Malawi}

C. Pfaff ${ }^{1}$, V. Singano ${ }^{1}$, H. Akello ${ }^{1}$, A. Amberbir ${ }^{1}$, D. Garone ${ }^{1}$, J. Berman $^{1}$, A. Kwekwesa ${ }^{1}$, A. Matengeni ${ }^{1}$, C. Speight ${ }^{3}$, T. Allain ${ }^{2,4}$, J. van Oosterhout ${ }^{1,2}$

1. Dignitas International, Zomba, Malawi

2. Department of Medicine, College of Medicine, University of Malawi,

Blantyre, Malawi

3. Lighthouse Trust, Lilongwe, Malawi

4. Independent consultant

\section{Introduction}

ART programs represent the first large scale chronic disease systems in Africa and can be leveraged to manage the growing burden of non-communicable disease (NCD).

\section{Methods}

Tisungane HIV clinic at Zomba Central Hospital has 6550 patients receiving treatment. In October 2015 a model of integrated HIVNCD care was developed. Clinical protocols for hypertension and diabetes screening and treatment were developed combining international literature and National Treatment Guidelines. All HIV clinic staff $(n=27)$ were trained during 2 two-day sessions. Blood pressure was measured on adults at every visit and random blood glucose every two years. If initial blood pressure was raised, it was repeated at that visit and confirmed on a subsequent visit before hypertension was diagnosed. Only patients with stage 2 hypertension (systolic $>160$ and/or diastolic $>100$ ) or those with stage I hypertension and cardiovascular risk factors were given antihypertensive drug treatment. If random glucose was high, it was confirmed with a fasting glucose, using WHO thresholds. All measurements were done by lay health workers and expert clients who received stipends. Combined ART-NCD treatment was provided by clinical officers, uncomplicated ART-only care by nurses.

\section{Results}

In the first six months $>6,500$ adults and children attended HIV care, of whom 5864 had $\geq 1$ blood pressure recorded and 3,803 had $\geq 1$ glucose reading measured. Five expert clients/lay health workers were employed for the additional care burden. This was manageable as the prevalence rates of newly diagnosed stage 2 hypertension and diabetes were low. The less-flexible vertical nature of the ART system made the design of integrated tools including electronic data capture challenging. Process mapping and patient flow were utilized to decrease missed screening opportunities. Integration of NCD care increased workload and consultation time.

\section{Conclusions}

Screening and treatment of hypertension and diabetes in single "one-stop" HIV-NCD clinic appears feasible.
C3 Attitudes and perceptions of pregnant women towards HIV 'opt out' testing in the context of Option B+ policy, QECH, Malawi

\section{Ruby Tionenji Ng'ong'ola, Lucinda Manda-Taylor}

College of Medicine, University of Malawi, Blantyre, Malawi

\section{Introduction}

HIV and AIDS continue to be the number one disease with the highest mortality. In 2012 it accounted for an estimated 1.6 million deaths. The prevalence of HIV and AIDS in sub-Saharan Africa currently stands at 4.7\% (15 - 49 of age) as of 2012. It is also estimated that $10 \%$ of the new HIV and AIDS infections in Malawi is acquired through mother to child transmission every year. There is a global effort to eliminate vertical transmission of new HIV infections. One component of this effort can be achieved through the adoption of efficacious prevention of mother to child transmission (PMTCT) programs. Bearing this in mind, Malawi, in 2011 began a new PMTCT program termed Option B+. However, Option B + is not without its weaknesses, ethical concerns are raised because most women are initiated on ARV treatment the same day they are tested for HIV, without providing proper informed consent.

\section{Objectives}

The main objective of this study was to describe women's knowledge, perceptions and attitudes on HIV testing and Option $\mathrm{B}+$. It also described women's understanding of informed consent during HIV testing and the implementation of Option B+.

\section{Methods}

This study was a cross sectional study utilizing qualitative research methods that took place at antenatal care (ANC) clinic, Queens Elizabeth Central Hospital (QECH). Data was collected through 15 in-depth interviews (IDIs) and 4 non participatory observation sessions of pregnant women that had undergone pre-information sessions, HIV testing and were enrolled on Option B+.

\section{Results}

It is promising to note that all women attending ANCs acquire knowledge on the concepts of HIV and AIDS and its mode of transmission and PMTCT polices from health workers at the clinic. The study participants also mentioned that they have understood the importance of HIV testing and Option $\mathrm{B}+$. However, interestingly, almost all the study respondents revealed that they did understand properly about informed consent and were not consented when it came to HIV testing and Option B+ programme.

\section{Conclusions}

This study demonstrated key findings that address how women perceive, experience and understand HIV testing within the context of PMTCT. Unfortunately, Option B+ negates women's rights to make voluntary decisions about their own healthcare. As a programme, Option B + is being delivered in an approach that makes HIV testing seem mandatory, thus ignoring the fact that women have a choice. In fact, the very name itself, Option B+, suggests that there are other options.

\section{C4 Understanding factors that shape community engagement in health research}

Deborah Nyirenda $^{1,2,3}$, Kate Gooding, ${ }^{2,3}$, Bertie Squire ${ }^{2}$, Chiwoza Bandawe $^{1}$, Salla Sariola ${ }^{4}$, Nicola Desmond ${ }^{2,3}$

1. College of Medicine, University of Malawi, Blantyre, Malawi

2. Liverpool School of Tropical Medicine, Liverpool, United Kingdom

3. Malawi-Liverpool-Wellcome Trust Clinical Research Programme, 
Blantyre, Malawi

4. Nuffield Department of Population Health, University of Oxford, Oxford, United Kingdom

\section{Introduction}

Community engagement is increasingly promoted in international research guidelines to improve: dialogue between researchers and communities and ethical research practice. There is however no widely agreed definition of community engagement leading to various interpretations. Limited evidence exists on effective approaches of engaging communities in two-way dialogue particularly in settings with low health literacy. Our research seeks to examine factors that shape the engagement process.

\section{Methods}

This paper draws from a wider study investigating the purpose, relevance and benefits of community engagement in health research using a qualitative research design. Three research projects were purposively selected as ethnographic case studies from two research institutions in Malawi. Participant observation was used to understand unarticulated aspects of community engagement in health research. In-depth interviews and focus group discussions were used to understand community engagement as experienced by stakeholders in research.

\section{Results}

Although community engagement is promoted in the literature to support two-way dialogue between researchers and communities, findings suggest that it is seen by stakeholders as a means of informing communities about research. We noted that community engagement practices were shaped by interactions between multiple stakeholders involved in research, their diverse agendas for engagement, communication approaches, interactions with other service providers in a given context, and other socio cultural factors. Despite having an aim of informing, a majority of research participants still had challenges to understand health research, raising concerns on informed consent processes.

\section{Conclusions}

We conclude that community engagement is used as a means of informing communities about research which does not match the ideals in the literature. This understanding is shaped by a number of social cultural factors. An understanding of these factors will help to design community engagement approaches that will promote meaningful dialogue in a particular context.

\section{C5 A comprehensive knowledge translation approach to improve the screening and treatment of hypertension in people living with HIV in Malawi}

Austrida Gondwe ${ }^{1}$, Josh Berman ${ }^{1}$, Collins Mitambo ${ }^{2}$, Shiraz $\mathrm{Khan}^{3}$, Beatrice L.Matanje-Mwagomba ${ }^{2}$, Chiyembekezo Kachimanga ${ }^{4}$, Emily Wroe ${ }^{4}$, Alemayehu Amberbir ${ }^{1}$, Moffat Nyirenda $^{5}$, Sam Phiri ${ }^{6}$, Tom Heller ${ }^{6}$, Salem Gugsa ${ }^{6}$, Joe Gumulira ${ }^{6}$, Colin Phaff ${ }^{1}$, Jean Baptiste Sagno ${ }^{7}$, Daniela Garone ${ }^{1}$, Joep J van Oosterhout $^{1,8}$, Damson Kathyola ${ }^{2}$

1. Dignitas International, Zomba, Malawi

2. Ministry of Health, Lilongwe, Malawi

3. UNC Project-Malawi, Lilongwe, Malawi

4. Partners in Health, Neno, Malawi

5. Malawi Epidemiology and Intervention Research Unit, Lilongwe, Malawi

6. Lighthouse Trust, Lilongwe, Malawi

7. DREAM Project, Blantyre, Malawi

8. Department of Medicine, College of Medicine, University of Malawi, Blantyre, Malawi

\section{Introduction}

In Malawi, recent studies show an adult hypertension burden of between $24 \%-46 \%$ with a large majority of individuals unaware of their hypertension. The prevalence among people living with HIV (PLWHA) also appears to be high. To support effective uptake of evidence into national health policies, Malawi has formed a knowledge translation platform (KTPMalawi) with support from WHO's Evidence-Informed Health Policy Network.

\section{Methods}

Led by the Ministry of Health's Department of Research, a steering committee prioritized the formation of a community of practice $(\mathrm{CoP})$ of policymakers, researchers, implementers and civil society members, to improve screening and treatment of hypertension in PLWHA. Members of the CoP were trained to develop a systematic evidence brief for policy by global KT experts. This evidence brief synthesized the best available evidence on the size and underlying factors of the problem, four policy options to address the problem, and examined potential implementation barriers. This brief was used as the primary input into a national policy dialogue.

\section{Results}

Dialogue participants concluded that:

1. More evidence was needed prior to recommending a policy direction.

2. Further research on non-communicable diseases (NCD) - HIV integration was warranted. Four geographically and programmatically diverse programs have been developed within Malawi and are implementing one of four proposed policy options:

1. Integrating the screening and treatment of hypertension into existing HIV clinics

2. Hypertension screening within HIV clinics and subsequent referral

3. Development of a comprehensive chronic care clinic model

4. The addition of hypertension screening and referral into community-based HIV activities

KTP Malawi has led a coordinated approach to align the programs' monitoring and evaluation frameworks to allow for meaningful comparisons on program outputs and outcomes.

\section{Conclusions}

KTP Malawi represents a viable model for engaging diverse stakeholders in evidence synthesis and policy development, ultimately feeding high quality evidence into ongoing policy discussions.

\section{C6 Diagnostic and prescribing practices and care seeking for uncomplicated malaria in under- five children in rural Chikwawa, Malawi - A health facility survey}

Alinune N. Kabaghe ${ }^{1,2}$, Mphatso D. Phiri ${ }^{3,4}$, Kamija S. Phiri ${ }^{2}$, Dianne J. Terlouw ${ }^{4}$, Michele van Vugt ${ }^{1}$

1. Academic Medical Centre, University of Amsterdam, Amsterdam, the Netherlands

2. College of Medicine, University of Malawi, Blantyre, Malawi

3. Queen Elizabeth Central Hospital, Blantyre, Malawi

4. Malawi-Liverpool Wellcome Trust Clinical Research Programme,

Blantyre, Malawi

\section{Background}

Malaria accounts for over a third of under-five outpatient visits in public health facilities in Malawi. Prompt and appropriate case management of malaria is key in reducing severity, burden and transmission of malaria. The Malawi National Malaria Treatment 
Guidelines recommend the use of malaria Rapid Diagnostic Tests (mRDT) to confrim cases before treatment with first-line antimalarial lumefantrine-artemether (LA). There is sparse data on delays in care seeking and prescriber adherence to national case management guidelines in rural Chikhwawa, an area of high malaria transmission.

\section{Methods}

We conducted a cross-sectional health facility survey and exit interviews with guardians of under-five febrile patients in health centres surrounding the Majete Wildlife Reserve in Chikhwawa to assess management of uncomplicated malaria in children and guardian promptness to seek care.

\section{Results}

We surveyed six health centres and conducted 120 exit interviews. Four facilities reported RDT or LA stock outs in the previous month. Seventy-one of $120(59.1 \%)$ guardians with febrile children presented within 24 hours of symptoms onset. Sixty-two of 120 (51.7\%) were RDT +, 45.8\% RDT- and $2.5 \%$ not tested. All RDT+ patients received an anti-malarial drug; two patients without an RDT + test received antimalarials. Among the cases treated, 72.6\% received LA, $27.3 \%$ received other antimalarials. For LA recipients, $28.9 \%$ were inappropriately dosed.

\section{Conclusions}

Findings suggest high health worker adherence to RDT result, but drug stock outs result in prescription of un-recommended anti-malarial drugs. Also, patients are frequently prescribed inappropriate doses of LA. Suboptimal dosing may contribute to spread of drug resistance.

\section{C7 Molluscicidal Properties of Sphenostylis marginata Tubers}

Moir Theu $^{1}$, Sosten Chiotha ${ }^{2}$, Chikumbusho Kayira ${ }^{1}$

1. University of Livingstonia, Livingstonia, Malawi

2. Leadership for Environment and Development (LEAD) Southern and Eastern Africa, Zomba, Malawi

\section{Introduction}

The increase in water development projects may increase the risk of schistosomiasis transmission in Malawi. One of the cheapest and environmentally friendly methods of preventing this transmission is the use of molluscicides derived from plants sources. Sphenostylis marginata is a local edible plant whose tuberous roots possess fungicidal, piscicidal, nutritional and medicinal properties. This study was conducted to test the efficacy of aqueous root extracts of $S$. marginata against Bulinus globosus, a fresh water vector snail species that hosts the urinary schistosomiasis parasite.

\section{Methods}

Plant tubers were collected for the dry and wet seasons. One hundred and sixty eight adult snails, with shell diameter of $9.5 \pm$ $1.0 \mathrm{~mm}$, were then subjected to aqueous preparations of powdered tubers in $24 \mathrm{~h}$ static bioassays. Seven nominal concentrations, including the control, were prepared from each sample and 12 snails were subjected to triplicates of each concentration. Snail mortality data was analyzed by probit method in SPSS to obtain $\mathrm{LC}_{50}$ values. The paired sample T-test was also conducted on the data to test the difference in efficacy of the extracts due to seasonal variation in tuber collection. Physiological changes were recorded as observed.

\section{Results}

Probit analysis showed that the wet season root powder extracts were the most lethal preparation with a corresponding $24 \mathrm{~h} \mathrm{LC}_{50}$ value of $297 \mathrm{mg} / \mathrm{L}$ compared to $367 \mathrm{mg} / \mathrm{L}$ for the dry season. There was a significant difference $(\mathrm{p}<0.05)$ between the wet and dry season samples. Physiologically, exposed snails exhibited escape behaviour, mucus excretion, hemorrhage and discoloration of the foot base.

\section{Conclusions}

The study has shown that locally available plants such as $S$. marginata have a potential to play a critical role in schistosomiasis snail control in Malawi.

\section{C8 Health services' response to harmful use of alcohol in Malawi: Health care staff regard towards management of alcohol use disorders (AUDs)}

\section{$\underline{B e a t r i c e ~ M w a g o m b a ~}{ }^{1}$, Alex Baldacchino, William Stones,} Moffat Nyirenda,

1. Joint Program in Global Health Implementation, College of Medicine, University of Malawi, Blantyre, Malawi \& University of St Andrews, Fife, United Kingdom

\section{Introduction}

Harmful use of alcohol is one of major risk factors for chronic non-communicable diseases (NCDs). This study aimed at assessing available health care services and staff perceptions for managing patients with alcohol use disorders (AUDs) relative to other NCDs in Malawi.

\section{Methods}

A cross-sectional study using mixed methods in 9 purposively sampled health facilities, including specialised psychiatric hospitals, general tertiary and secondary level hospitals. Data were collected using structured in-depth interviews with site incharges and a self-administered Medical Condition Regard Scale (MCRS) that measured staff regard for working with patients with different NCDs including alcohol use disorders, diabetes mellitus, hypertension, other cardiovascular diseases and mental health disorders. Complementary data from multidisciplinary focus group discussions (FGDs) were also collected. Mean MCRS scores by condition, followed by score comparisons using Analysis of Variance (ANOVA) were conducted in SPSS statistical package. Qualitative data transcripts were coded and analyzed using NVivo by a deductive approach.

\section{Results}

Only the specialised psychiatric hospitals and mental health clinics had some form of guidelines for managing AUDs. Tests used for identification or diagnosis of AUDs were various including: CAGE, SCID and the DSM-IV alcohol module. Overall, regard for working with AUDs was significantly lower than regard for other NCD conditions. Lack of standardized AUD management protocols, inservice training and appropriate medicines (detoxifiers) were the commonly perceived barriers to management of AUDs.

\section{Conclusions}

Establishing a standardised system for identification of AUDs can potentially enhance staff competencies for management of harmful use of alcohol in Malawi. 
C9 Risk factors for reported asthma among urban and ural adults in Malawi

\author{
Abena S. Amoah ${ }^{1}$, A.C. Crampin, S. Geis, A. Amberbir, N. \\ Kayuni, C. Musicha, M.J. Nyirenda. \\ 1. Malawi Epidemiology and Intervention Research Unit, Lilongwe, \\ Malawi
}

\section{Introduction}

The incidence of asthma is on the increase in low-and middle income countries with a number of factors being implicated in this rise. The objective of the study was to investigate risk factors for self-reported asthma in urban and rural adults in Malawi in preparation for future interventions.

\section{Methods}

We conducted a cross-sectional study on the burden and risk factors for non-communicable diseases among urban adults in Lilongwe City and rural adults in Karonga district. Trained interviewers collected data on self-reported asthma diagnosis and asthma symptoms as main outcomes. Information was also collected on body mass index (BMI), demographic characteristics, socioeconomic status, environmental exposures and other chronic disease co-morbidities for our risk factor analysis.

\section{Results}

Data on a total of 27,820 participants $(50.9 \%$ urban and $49.2 \%$ rural) were included in the analysis. Preliminary results show that about $5.1 \%$ of urban subjects reported a history of diagnosed asthma compared to $4.5 \%$ of rural participants. In urban women only, we observed positive associations between obesity (defined as BMI >30) as well as indoor fire exposure and self-reported asthma When it came to wheeze in the past 12 months, being underweight (BMI <18) compared to having a normal BMI (18-25) was positively associated with wheeze in rural women and urban men but not in urban women or rural men.

\section{Conclusions}

Although physical examinations are needed to validate the selfreported asthma diagnosis, the study provides indications that there is a role for body mass index and environmental exposures in the manifestations of diagnosed asthma and related symptoms in Malawi. There are also indications of gender and urban-rural differences in asthma risk factors. Health programmes would have to take into account such differences in the planning of programmes to tackle the burden of asthma in Malawi.

\section{C10 Impact of active tracing on overall survival estimates among children with lymphoma in Lilongwe}

Christopher Stanley ${ }^{1}$, Kate Westmoreland ${ }^{1,5}$, Salama Itimu ${ }^{1}$, Ande Salima ${ }^{1}$, Toon Van Der Gronde ${ }^{1}$, Paula Ward ${ }^{1}$, Nader ElMallawany $^{2}$, Peter Wasswa ${ }^{2,3}$, Idah Mtete ${ }^{3}$, Mercy Butia ${ }^{3}$, Satish Gopal ${ }^{1,4,5}$

1. UNC Project-Malawi, Lilongwe, Malawi

2. Texas Children's Hospital, Houston, Texas, USA

3. Baylor College of Medicine Children's Foundation Malawi, Lilongwe, Malawi

4. University of North Carolina, Chapel Hill, North Carolina, USA

5. College of Medicine, University of Malawi, Blantyre, Malawi

\section{Introduction}

Lymphoma is the commonest pediatric cancer in Malawi. Survival estimates from sub-Saharan Africa are often based on short followup with significant loss to follow-up (LTFU). The magnitude of bias introduced by LTFU and opportunities to correct through active follow-up are uncertain.

http://dx.doi.org/10.4314/mmj.v28i4.9
Methods

Children with newly diagnosed lymphoma were enrolled June 2013-February 2016 in Lilongwe. Staging, supportive care and treatment were standardized, and children were actively traced using telephone and home visits. For comparison, follow-up time and overall survival (OS) were calculated from enrolment until death or LTFU using passive and active tracing respectively.

\section{Results}

121 children were enrolled with lymphoma during the study period: 100 (83\%) Burkitt lymphoma (BL) and 21 (27\%) Hodgkin lymphoma (HL). Median age was 10.1 years (interquartile range (IQR) 7.1-12.8), 84 (69\%) male, 85 (70\%) were stage III/IV, and 94 $(78 \%)$ had poor performance status. Using passive tracing, median follow-up time was 4.4 months IIQR 2.0-9.4; BL 3.9 months (IQR 1.9-8.0), HL 9.4 months (IQR 6.1-12.5)], and 12-month OS was 69\% [95\% CI 54-80; BL 63\% (95\% CI 44-77), HL 83\% (95\% CI 55-94)]. With active tracing, median follow-up increased to 10.8 months [IQR 6.2-20.6; BL 10.4 months (IQR 5.3-18.4), HL 11.6 months (IQR 9.4-20.8)], and 12-month OS decreased to 44\% [95\% CI 34-54; BL 38\% (95\% CI 27-48), HL 74\% (95\% CI 48-89)].

\section{Conclusions}

Passive follow-up significantly overestimated OS and underestimated mortality for pediatric lymphoma in Malawi. Active tracing increases follow-up and minimizes bias in OS estimation, with low cost and high implementability.

\section{C11 Focal adhesion proteins, vinculin and integrin $\beta 5$, during early pregnancy in rat uterine epithelial cells: Anastrozole favors their normal distribution}

\author{
Anthony Mwakikunga ${ }^{1,2}$, Lynne Schepartz ${ }^{2}$, Margot Hosie $^{2}$ \\ 1. Department of Basic Medical Sciences, College of Medicine, \\ University of Malawi, Blantyre, Malawi \\ 2. School of Anatomical Sciences, University of the Witwatersrand \\ Medical School, Johannesburg, South Africa
}

\section{Introduction}

An alternative superovulator to replace clomiphene citrate is needed as clomiphene citrate is associated with low pregnancy rates. Anastrozole is an effective superovulator, but it has not been well researched. In order to determine the effectiveness of anastrozole as a superovulator and to compare it with clomiphene citrate in similar situations, this study ascertained the effects of these drugs on the expression of the focal adhesion proteins, vinculin and integrin $\beta 5$, which are uterine receptivity markers, in the uterine epithelial cells of day 1 and day 6 pregnant Wistar rats.

\section{Methods}

Thirty five mature female rats were randomly divided into 7 dose groups of 5 rats each: $1 \mathrm{mg} / \mathrm{kg}, 10 \mathrm{mg} / \mathrm{kg}, 15 \mathrm{mg} / \mathrm{kg}$ and $25 \mathrm{mg} / \mathrm{kg}$ of body weight anastrozole; $1.25 \mathrm{mg} / \mathrm{kg}$ of body weight CC, saline and untreated groups. Rats in pro-oestrus received the treatment regimes and were caged overnight with males. Pregnant rats were sacrificed on day 6 around the time of implantation. Implantation sites were recorded. JMP was used for statistical comparisons of means of implantation sites between treatment groups using Turkey-Kramer HSD. Differences were considered statistically significant when $p<0.05$.

\section{Results}

The results show that vinculin and integrin $\beta 5$ are co-localized at the base of the uterine epithelium at day 1 of pregnancy whereas at day 6 , they disassemble from the basal focal adhesions and co-localize and significantly increase their expression apically $(\mathrm{p}<0.0001)$. MMJ VOL 28 (4): December 2016 
Moreover, there is a significant difference in the protein expression levels of vinculin and integrin $\beta 5$ in uterine luminal epithelial cells between untreated (control) and chlomiphene citrate treated rats $(p<0.0001)$, anastrozole and chlomiphene citrate treated rats at day $6(\mathrm{p}<0.0001)$ suggesting the interpretation that anastrozole seems to enhance their expression in order to perhaps assist in the implantation process of the blastocyst. The immunofluorescence experiments agree with the vinculin and integrin $\beta 5$ gene expression findings in which at day 6 of pregnancy vinculin and integrin $\beta 5$ gene expression are significantly up-regulated in uterine luminal epithelial cells in the anastrozole treated group relative to the calibrator sample $(\mathrm{p}<0.0001)$.

\section{Conclusions}

These findings suggest that anastrozole is implantation friendly. The effect of anastrozole in the level of estrogen $\left(\mathrm{E}_{2}\right)$ and progesterone $(\mathrm{P})$ may change the $\mathrm{E}_{2} / \mathrm{P}$ ratio at day 6 of pregnancy, suggesting its effect on endometrial maturation and therefore improving receptivity.

\section{C12 Inhibitory geostatistical designs for spatial prediction taking account of uncertain covariance structure}

\author{
Michael G. Chipeta ${ }^{1,2,3}$, Dianne J. Terlouw ${ }^{2,3,4}$, Kamija S. Phiri ${ }^{2}$, \\ Peter J. Diggle \\ 1. Lancaster Medical School, Lancaster University, Lancaster, United \\ Kingdom \\ 2. College of Medicine, University of Malawi, Blantyre, Malawi \\ 3. Malawi-Liverpool-Wellcome Trust Clinical Research Programme, \\ Blantyre, Malawi \\ 4. Liverpool School of Tropical Medicine, Liverpool, United Kingdom
}

\section{Introduction}

The problem of choosing spatial sampling designs for investigating unobserved spatial phenomenon arises in many contexts, for example in identifying households to select for a prevalence survey to study disease burden and heterogeneity in a study region.

\section{Methods}

We studied randomised inhibitory spatial sampling designs to address the problem of spatial prediction whilst taking account of the need to estimate covariance structure. Two specific classes of design are inbibitory designs and inbibitory designs plus close pairs. In an inhibitory design, any pair of sample locations must be separated by at least an inhibition distance $\delta$. In an inhibitory plus close pairs design, sample locations in an inhibitory design with inhibition distance $\delta$ are augmented by locations each positioned close to one of the randomly selected ocations in the inhibitory design, uniformly distributed within a disc of radius.

\section{Results and conclusions}

I present simulation results for the Matern class of covariance structures. When the nugget variance is non-negligible, inbibitory plus close pairs designs demonstrate improved predictive efficiency over designs without close pairs. I illustrate how these findings can be applied to the design of a rolling Malaria Indicator Survey that forms part of an ongoing large-scale, five-year malaria transmission reduction project in Majete perimeter, southern Malawi.

\section{MATERNAL AND CHILD \\ HEALTH}

\section{D1 Assessment of the quality of care in maternal waiting homes (MWHS) in Mulanje District, Malawi}

\section{Leticia Chimwemwe Suwedi}

School of Public Health and Family Medicine, College of Medicine, University of Malawi, Blantyre, Malawi

\section{Introduction}

Maternal Mortality Ratio (MMR) in Malawi remains high at 675 deaths per 100,000 live births, primarily due to pregnant women's lack of access to skilled birth care. Although Malawi established Maternal Waiting Homes (MWHs) to improve access to skilled labour, the quality of care provided in the homes has received limited assessment. This study assessed the clients' and providers' perceptions on the quality of care in MWHs in Mulanje district.

\section{Methods}

We conducted a descriptive qualitative study in 3 MWHs in Mulanje district, Malawi from June 2015 to June 2016. We conducted a non-participatory observation using a checklist on the facilities, 6 face to face in depth interviews with health providers and 4 Focus group discussions (FGDs) with 33 pregnant women admitted for more than 48 hours in MWHs. We digitally recorded all FGDs and IDI and simultaneously transcribed and translated verbatim into English. Data were analysed using thematic analysis.

\section{Results}

There were mixed reactions towards the quality of care in the MWHs. The layout and care provided varied across the facilities. There were no standards to guide provision of health services in MWHs. Factors that positively influenced quality of care were quite environment at the MWH and midwifery services while lack of cooking spaces, 24 hours nursing care, recreation services, absence of food and sleeping on the floor negatively affected perception of quality.

\section{Conclusions}

There is a need to develop MWHS guidelines to optimize provision of standardised quality services.

\section{D2 Health outcomes of babies born before arrival at the hospital: A case study of Bwaila Maternity Hospital, Lilongwe, Malawi}

\section{Eunice Nahache ${ }^{1}$, Adamson Muula ${ }^{2}$}

1. Kamuzu College of Nursing, University of Malawi, Lilongwe, Malawi 2. College of Medicine, University of Malawi, Blantyre, Malawi

\section{Introduction}

Despite Malawi government's effort to support women deliver in health facilities, some women deliver babies before arriving (BBA) at health facility. Studies have shown higher case fatality rate among neonates of BBA than babies delivered at health institutions. Lack of Essential New born Care (ENC) exposes BBAs to infection and death. The study assessed health outcomes of BBA at Bwaila Maternity Hospital in Lilongwe from January 2013 to December 2014

\section{Methods}

Cross sectional study design utilizing qualitative and quantitative methods was used. Secondary data from Lilongwe District Health 
Information Management System and 303 admission case files of mothers from January 2013 to December 2014 were used.

\section{Results}

The prevalence of BBA was 8.8\% in 2013 and $7.9 \%$ in 2014. About $70.6 \%$ of BBA were admitted in nursery ward due to several complications and $18 \%$ of BBAs died. The Majority of women who had BBAs were from urban areas. Some of the characteristics associated with BBA outcome included: baby weight, parity, gestation period, age and, education level. Health system factors reported to contributing to BBA include: poor referral systems, negative attitude of health workers towards women in labour and inadequate information on labour and delivery.

\section{Conclusions}

We have shown that majority of BBAs were admitted at the hospital with several complications. Among these BBA, low birthweight, birth asphyxia and prematurity were the main causes of death. Poor referral system, failure of health workers to convey adequate information on labour and delivery and negative attitude of staff were some of health system factors contributing to BBA.

\section{D3 Persistent vaccine type carriage of Streptococcus pneumoniae four years after introducing 13 -Valent pneumococcal conjugate vaccine in a $3+0$ schedule in Malawi}

T.D. Swarthout ${ }^{1}$, A. Kamng'ona ${ }^{2}$, T. S. Mwalukomo ${ }^{2}$,

D. Everett ${ }^{1}$, N. Bar-Zeev ${ }^{1}$, T. Chipasula ${ }^{1}$, K. Malisita ${ }^{2}$, C. Mwansambo $^{3}$, M. Banda 4 , J. Hinds ${ }^{5}$, K.A. Gould ${ }^{5}$, N. French ${ }^{6}$, R.S. Heyderman $^{7}$

1. Malawi-Liverpool-Wellcome Trust Clinical Research Programme, Blantyre, Malawi

2. College of Medicine, University of Malawi, Blantyre, Malawi

3. Ministry of Health, Lilongwe, Malawi

4. Ministry of Education, Blantyre, Malawi

5. St George's - BUGS Bioscience, University of London, London,

United Kingdom

6. Division of Infection \& Immunity, University College London,

London, United Kingdom

\section{Introduction}

In 2011 Malawi introduced PCV13, at 6, 10 and 14 weeks of age. We explore whether there are under-recognized reservoirs of pneumococcal carriage where vaccine serotypes (VT) and diseasecausing non-vaccine serotypes (NVT) persist.

\section{Methods}

Cross-sectional nasopharyngeal carriage surveys over 4 years in Blantyre, Malawi, commencing June 2015. Recruitment includes PCV13-vaccinated and -unvaccinated healthy, HIV-uninfected children aged 3-10 years, using stratified random sampling of households and schools; and adults aged 18-40 receiving ART. Swabs in STGG were cultured on SB-Gentamicin. Pneumococcal serotyping used a 13-valent latex kit (SSI, Denmark). Multiple serotype carriage (MSC) investigation used molecular serotyping by microarray (BUGS Bioscience, UK). This was in collaboration with ongoing pneumococcal disease surveillance.

\section{Results}

Analysis includes 629 vaccinated (aged 3-4) and 473 unvaccinated children (aged 5-10) and 400 adults. PCV13 coverage is $>85 \%$ amongst age-eligible. By latex, VT and NVT carriage among vaccinated children is respectively $22.4 \%(95 \%$ CI: $18.3,27.1)$ and $59.2 \%(53.0,67.2)$; unvaccinated children: $25.3 \%(20.9,31.1)$ and $41.3 \%(35.7,47.0)$; adults: $13.9 \%(9.8,18.8)$ and $28.6 \%(23.0,34.7)$. Serotype-1 represents respectively $5.2 \%, 12.0 \%$ and $7.0 \%$ of VT carriage. Among a representative subset of carriers, MSC is $55.7 \%$ (162/291) amongst children aged 3-6 years. Microarray increased prevalence of detectable VT carriage by $35.6 \%$.

http://dx.doi.org/10.4314/mmj.v28i4.9
Conclusions

While significantly reducing burden of pneumococcal disease, four years after PCV13 introduction there is substantial VT nasopharyngeal carriage. This suggests the current schedule has, to date, had limited indirect effect. Review of schedules to optimise total population impact in high carriage, high disease-burden countries such as Malawi is warranted.

\section{D4 Physical intimate partner violence is associated with adverse birth outcomes}

\author{
$\underline{\text { N. Rao }}^{2}$, A. Turner ${ }^{2}$, B. Harrington ${ }^{4}$, P. Nampandeni ${ }^{3}$, V. Banda ${ }^{3}$, \\ A. Norris ${ }^{1}$
}

1. Division of Epidemiology, College of Public Health, The Ohio State University, Columbus, Ohio, USA

2. College of Medicine, The Ohio State University, Columbus, Ohio, USA

3. Child Legacy International, Umoyo wa Thanzi Research Program, Lilongwe District, Malawi

4. Department of Epidemiology, University of North Carolina, Chapel Hill, North Carolina, USA

\section{Introduction}

Intimate partner violence (IPV) has been consistently linked to increased rates of miscarriage, stillbirth, prematurity, and neonatal death in high-resource settings. Although IPV is highly prevalent in sub-Saharan Africa, few studies have characterized the association between IPV and adverse birth outcomes in the region.

\section{Methods}

We use data from the baseline survey of a cohort study of sexual and reproductive health decision making in rural Malawi to examine the association between physical IPV experienced by women (with their current husband) and history of adverse birth outcomes - miscarriages, stillbirths, and neonatal deaths - among married Malawian women.

\section{Results}

Among women in our analysis population $(n=792)$, physical IPV was prevalent $(21 \%)$. We observed no significant differences in age $(p=0.98)$, income $(p=0.64)$, and education $(p=0.81)$ between women with a history of IPV and women without a history of IPV. Women who reported experiencing physical IPV with their husbands were significantly more likely to have a history of adverse birth outcomes in unadjusted (prevalence ratio (PR): 1.23; 95\% confidence interval (CI): 1.08, 1.41) and adjusted (adjusted PR (aPR): 1.19; 95\% CI: 1.01, 1.40) analyses.

\section{Conclusions}

Physical IPV was prevalent among married Malawian women and was significantly, albeit modestly, associated with adverse birth outcomes. Though temporality is a limitation in these data - we were not able to determine the timing of IPV in relation to adverse birth outcomes - confidential screening for physical IPV and prompt referral to support services could have added benefit for improving maternal and child health in this setting. 
D5 Quality of facility-based family planning services for adolescents in Malawi: Findings from a national census of health facilities

\author{
Vinitha Jayachandran $^{1}$, Gertrude Chapotera ${ }^{1}$, William Stones ${ }^{1,2,3}$ \\ 1. School of Public Health and Family Medicine, College of Medicine, \\ University of Malawi, Blantyre, Malawi \\ 2. Department of Obstetrics and Gynaecology, College of Medicine, \\ University of Malawi, Blantyre, Malawi \\ 3. School of Medicine, University of St Andrews, Fife, United Kingdom
}

\section{Introduction}

Fertility remains high among Malawian adolescents despite extensive investment in 'youth friendly' services. We examined provision and experience of care in facility-based family planning services. We hypothesised that the quality of services might be influenced by the age of the client.

\section{Methods}

We used data from the Service Provision Assessment survey 2013-14, a census of all formal health facilities in the country. The inclusion criterion was that the client's age was recorded in the data set, which gave a weighted total of 1388 observations of consultations, reflecting provision of care, and client exit interviews, reflecting experience of care.

\section{Results}

The youngest clients had twice the odds of reporting a better experience of care compared to clients aged 26 and older (OR $2.03,95 \%$ CI 1.15 to $3.54, \mathrm{P}=0.013)$. The standard of observed provision was low, typically with half or more of the mandated elements of care omitted. Compared with older clients, provision of care was slightly better for adolescents. Care was better in NGO facilities but worse in clinics compared to hospitals and also for clients seen by health surveillance assistants compared to those seen by a clinician.

\section{Conclusions}

Quality of care for adolescents attending facility-based family planning services was slightly better than for older clients, but this is overshadowed by the finding of a low standard of care overall. Health system strengthening, especially at the clinic level, is a policy and programming priority that will contribute to adolescent reproductive health in Malawi.

D6 Validity and acceptability of self-collected samples for HPV testing in rural Malawi: Findings from a clinic-based feasibility Study

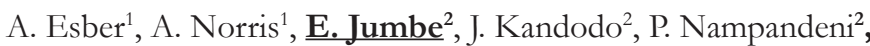 \\ A.N. Turner
}

1. Division of Epidemiology, The Ohio State University, Columbus, Ohio, USA

2. Child Legacy International, Umoyo wa Thanzi Research Program, Lilongwe District, Malawi

3. College of Medicine, The Ohio State University, Columbus, Ohio, USA

\section{Introduction}

The WHO recently endorsed HPV testing as a cervical cancer screening method in countries without established programs. Self-collection for HPV testing may be an effective way to expand screening to hard to reach women. Our objective was to assess the feasibility, validity, and acceptability of self-collection for HPV testing in a population of care-seeking, unscreened women in rural Malawi.

\section{Methods}

We enrolled women reporting to a rural Malawian clinic from January -August 2015. Participants were offered the option to self- collect a vaginal sample and the study clinician collected a cervical sample for HPV testing. Using the clinician-collected sample as the reference standard we calculated a kappa statistic, sensitivity, and specificity by hr-HPV type. Participants also received a brief survey assessing acceptability of the procedure.

\section{Results}

Among the 193 enrolled women, 22\% had any hr-HPV. Comparing self- and clinician-collected samples for HPV testing, we found generally high agreement $(x=0.60-0.90)$ and high specificity $(98 \%$ $100 \%$ ), but varied sensitivity $(50 \%-91 \%)$ for different types of hr-HPV. We also found that self-collection was acceptable, with $98 \%$ of women reporting it was easy to do and 98\% reporting willingness to do so again.

\section{Conclusions}

Our findings demonstrate that self-collection of samples for HPV testing is a feasible and acceptable method of cervical cancer screening in this rural Malawian population. High agreement between the self- and clinician-collected samples, and high levels of acceptability among women in the study suggest that selfcollection of vaginal samples for HPV testing may be effectively incorporated into screening programs among rural, largely unscreened populations.

\section{D7 Sensory evaluation of Mgaiwa-Moringa oleifera leaf powder porridge}

\section{R. Manda, A.M. Mwangwela}

Lilongwe University of Agriculture and Natural Resources, Bunda Campus, Lilongwe, Malawi

\section{Introduction}

Moringa powder is a potential locally sourced nutrition supplement to combat nutrient deficiencies in Malawi. The limiting factor to the use of Moringa is its unpalatable sensory properties which lead to decreased acceptability. In light of its high quality nutritional profile, there is a need to use Moringa, create a more palatable way to incorporate it into traditional Malawian meals. The study's objective was to analyze the effects of cooking method (Moringa powder added during cooking vs Moringa powder added on the plate after cooking) on sensory characteristics, develop and refine a recipe where Moringa powder is incorporated into mgaiwa porridge.

\section{Methods}

Moringa leaf powder was prepared according to AVARD, 2007. Mgaiwa and Moringa leaf powder were added in the ratios of 100:0, 95:5, 90:10, 85:15, 80:20 and 75:25. Sensory evaluation was done by using descriptive and consumer acceptance tests (Lawless and Heymann, 2010). SPSS was used to analyze data.

\section{Results}

Addition of Moringa leaf powder did not affect viscosity, stickiness, grittiness, and fermentation of porridge but increased its olive green colour, speckles, sourness, bitterness, nutty, burnt and Moringa aromas. Saltiness, sweetness, well-cooked taste, shininess and mgaiwa aroma were reduced. Addition of Moringa powder above $15 \%$ decreased acceptability of the porridge $(\mathrm{P}>0.05)$. Adding Moringa powder during cooking was the preferred method $(82 \%)$ as fermented aroma, olive green colour, bitterness and shininess were decreased and mgaiwa, nutty aromas, softness, sweetness and saltiness of the porridge were not affected.

\section{Conclusions}

Addition of Moringa powder to foods increased bitterness, Moringa and fermented aromas which reduced acceptability. Cooking reduced these unpalatable sensory characteristics making the porridge acceptable. 
D8 Pancreatic enzyme replacement therapy in children with severe acute malnutrition: A randomised controlled clinical trial

$\underline{\text { Rosalie H. Bartels }}^{1,2}$, Céline Bourdon ${ }^{3}$, Isabel Potani ${ }^{2}$, Brian Mhango $^{2}$, Deborah A. van den Brink ${ }^{4}$, John S. Mponda ${ }^{5}$, Robert H. Bandsma ${ }^{4,6}$, Michael Boele van Hensbroek ${ }^{1}$, Wieger P. Voskuij ${ }^{1,2}$

1. Global Child Health Group, Emma Children's Hospital, Academic Medical Centre, Amsterdam, the Netherlands

2. Department of Pediatrics and Child Health, College of Medicine,

University of Malawi, Blantyre, Malawi

3. Division of Gastroenterology, Hepatology and Nutrition, The

Hospital for Sick Children, University of Toronto, Toronto, Ontario,

Canada

4. Department of Pediatrics, Center for Liver, Digestive and Metabolic

Diseases, University of Groningen, University Medical Center

Groningen, Groningen, the Netherlands

5. Department of Pharmacy, College of Medicine, University of Malawi, Blantyre, Malawi

6. Department of Biomedical Sciences, College of Medicine, University of Malawi, Blantyre Malawi

\section{Introduction}

Severe acute malnutrition (SAM) is associated with high mortality rates which is often related to severe diarrhoea. Recent data indicate that most children with SAM suffer from exocrine pancreatic insufficiency (EPI). We aimed to assess the effect of pancreatic enzyme replacement therapy (PERT) in children with complicated SAM.

\section{Methods}

This randomized controlled trial was conducted at Queen Elizabeth Central Hospital in Blantyre, Malawi. Ninety children with SAM were randomly assigned to receive additional PERT for 28 days or standard nutritional rehabilitation feeds only. Primary outcome was the percentage of weight gain after 28 days. Secondary outcome measures were mortality after the intervention, improvement of EPI by increased FE-1 levels, and duration of admission.

\section{Results}

No significant difference $(\mathrm{p}=0.71$ ) was found in the percentage of weight gain after 28 days between the PERT group and the control group ( $15.2 \pm 11.3 \%$ and $14.3 \pm 8.1 \%$, respectively). Although not powered to detect such, mortality was significantly lower in the PERT group $(17.8 \%$ versus $40 \%, \mathrm{p}=0.035)$. Interestingly, the duration of admission until death (4.9 vs 4.5 days) was not significantly different $(p=0.250)$.

A significant increase in FE-1 was found after 28 days in both groups $(\mathrm{p}<.000)$. Duration of admission was equal in both groups $(\mathrm{p}=0.437)$.

\section{Conclusions}

Additional PERT therapy did not result in extra weight gain after 28 days in children with SAM. Mortality was lower in the PERT group suggesting a potential beneficial effect. Both groups showed an increase in pancreatic function (FE-1). These findings warrant further investigation in a larger cohort.
D9 Coverage, awareness and adoption levels of ecological sanitation latrines in Traditional Authority Mzukuzuku in Mzimba District

\author{
John L.Z. Nyirenda ${ }^{1}$, K. Phiri ${ }^{2}$, S.G. Simeza ${ }^{3}$
}

1. Malawi-Liverpool-Wellcome Trust Clinical Research Programme, Blantyre, Malawi

2. Chancellor College, University of Malawi, Zomba, Malawi

\section{Introduction}

Synod of Livingstonia Development Department (SOLDEV) started implementing community water and sanitation project in Mzimba district in 2002. Ecological sanitation promotes personal hygiene and use of human manure for fruits and crops production. The research was conducted to assess awareness, coverage and adoption levels of the ecological sanitation latrines in Traditional Authority Mzukuzuku in Mzimba District.

\section{Methods}

The assessment engaged cross-sectional and observatory approaches in the quantitative paradigm. The structured questionnaire was used to conduct interviews with the adults in the sampled villages; targeting heads of households.

\section{Results}

Many people; $92.3 \%$, heard about ecological sanitation while $45.6 \%$ had satisfactorily defined ecological sanitation in simple terms. The coverage of basic sanitation latrines is $58.9 \%$ and that of ecological sanitation is $25 \%$. Furthermore, the results showed that $33 \%$ of the households have ever used compost manure from the human faeces and $76.8 \%$ of the household accepts use of human manure for fruit and crop production.

\section{Conclusions}

The results suggest that awareness is not adequate and adoption levels are low. In brief, awareness has to be improved so that many people have adequate knowledge about ecological sanitation. It is also suggested that the promoters be added and accessibility to construction materials should be improved for promotion of coverage and adoption of ecological sanitation latrines in the district. Despite low adoption levels, the project contributed to introduction of Ecological sanitation latrines and improvement of sanitation standards and coverage of latrines in the impact communities.

\section{D10 Influence of plasma fatty acids on zinc dynamics and blood clotting}

Kondwani G.H. Katundu ${ }^{1,2}$, Fanuel Lampiao ${ }^{1}$, Claudia A. Blindauer $^{3}$, Alan J. Stewart ${ }^{2}$,

1. College of Medicine, University of Malawi, Blantyre, Malawi

2. School of Medicine, University of St Andrews, Fife, United Kingdom

3. Department of Chemistry, University of Warwick, Coventry, United Kingdom

\section{Introduction}

Zinc ions are a key regulator of haemostasis. The concentration of labile zinc in plasma is tightly regulated through binding to Human serum albumin (HSA). The zinc binding capacity of HSA is altered in disease conditions such as diabetes and cancer, which are also associated with increased plasma free fatty acid (FFA) levels; FFAs can allosterically disrupt binding of zinc to HSA. Zinc and cobalt share a binding site on HSA. Thus, the albumin cobalt binding (ACB) assay may be employed in assessing effects of FFAs on metal/zinc binding by HSA. Platelet aggregation experiments may also be useful to study effects of unregulated zinc in plasma on platelet aggregation.

Methods

The influence of FFAs of different chain lengths on cobalt-HAS 
binding was investigated by the ACB assay. In addition, a platelet aggregation assay using fresh platelet-rich-plasma (PRP) in 96-well format was established, optimised and the influence of $\mathrm{Zn}^{2+}$ on platelet aggregation was examined.

\section{Results}

Saturated fatty acids elicited concentration-dependent effects upon cobalt binding to HSA, with longer chain FFAs associated with greater perturbation of cobalt binding. Whilst addition of $10 \mu \mathrm{M}$ $\mathrm{ZnCl}_{2}$ to PRP caused an increase in platelet aggregation response using the optimised assay.

\section{Conclusions}

The results of the experiments are consistent with the hypothesis that elevated levels of FFA, as associated with certain disease states, may adversely influence haemostasis through mishandling of zinc and lead to the development of thrombotic complication.

\section{D11 Development of real time polymerase chain reaction for the detection of Salmonella in stool specimens}

\author{
Angeziwa Chirambo ${ }^{1,5}$, Tonney Nyirenda ${ }^{1,2}$, Ndaru Kaluwa ${ }^{1}$, \\ Chisomo Msefula $^{1,2}$, Sandra Molina ${ }^{5}$, Melita Gordon ${ }^{1,4}$ \\ 1. Malawi-Liverpool-Wellcome Trust Clinical Research Programme, \\ Blantyre, Malawi \\ 2. Department of Pathology, College of Medicine, Blantyre, Malawi \\ 3. Liverpool School of Tropical Medicine, Liverpool, United Kingdom \\ 4. University of Liverpool, Liverpool, United Kingdom \\ 5. London School of Hygiene and Tropical Medicine, London, United \\ Kingdom
}

\section{Introduction}

Salmonella is among the leading cause of bacteremia and death in sub Saharan African children. The burden of Salmonella in Africa and the link between Salmonella exposure within the gastrointestinal tract and blood stream is poorly understood in part due to lack of reliable diagnostic test for detection of Salmonella. Stool culture which is the gold standard for Salmonella detection is less sensitive and time consuming. In this study, we validated a RT-PCR test for Salmonella in stool specimens from a cohort of healthy children.

\footnotetext{
Methods

TTR and InvA primers were tested for Inclusivity and exclusivity, Specificity and sensitivity. The primers were also validated against stool culture for Salmonella. Salmonella exposure events in stool samples collected from a cohort of healthy children aged 6-18 months was also determined.
}

\section{Results}

Both TTR and InvA RT-PCR primers demonstrated 100\% inclusivity and between $87 \%$ and $94 \%$ exclusivity. Stool culture and RT-PCR (TTR and InvA) were able to determine Salmonella exposure event in 409 stool samples by $4.4 \%, 8.8 \%$ and $13.7 \%$ respectively. Using stool as the gold standard, specificity and sensitivity of TTR was $94.3 \%$ and $88.8 \%$, and for InvA it was $89.2 \%$ and $77.7 \%$ respectively.

\section{Conclusions}

TTR is more specific and sensitive than InvA but they both perform much better than stool culture. Cross reactivity of the primers is reduced by Selenite enrichment of the samples. The two primers can be used together as a diagnostic tool for surveillance studies.

\section{D12 Evaluation of a new tool for assessing pharmaceutical quality}

\section{Ibrahim Chikowe $^{1}$, Marya Lieberman ${ }^{2}$}

1. Faculty of Biomedical Science and Health Professions, College of Medicine, University of Malawi, Blantyre, Malawi

2. Department of Chemistry and Biochemistry, University of Notre Dame, Notre Dame, Indiana, USA

\section{Introduction}

Paper Analytical Devices (PADs) have given promising results on the rapid testing of medicines. These inexpensive paper cards can carry out multiple chemical colour tests to assist in routine quality control activities in communities that have limited access to expensive quality control procedures. This method is aimed at detecting suspicious medicines in the field that are then taken to the central laboratory for confirmatory tests using the gold standard method the HPLC.

\section{Methods}

In this preliminary study, we looked at the suitability of this tool on the medicines used in Malawi. Ten antibiotic products of different brands were collected and the PAD was used to classify their active ingredients by comparison to reference images. Next, we plan to carry out thin-layer chromatography (TLC).

\section{Results}

PAD results were comparable to the reference images, which supports the idea that they can work on a wide range of antibiotics from different sources.

\section{Conclusions}

The preliminary results show that the PADs can work for assessing pharmaceutical quality in Malawi. Further studies are underway now to sample more classes and brands of medicines (anti-HIV, anti-TB and antimalarial medicines) with the cooperation of the Pharmacy, Medicines and Poisons Board (PMPB). Field screening will be conducted in Malawi using packaging analysis, semiquantitative thin-layer chromatography (SQ-TLC) and a paper test card (the PADs) to assess drug quality, followed by confirmatory analysis using high pressure liquid chromatography (HPLC). The test results will be shared with the PMPB, the World Health Organization (WHO) and archived and visualized using fusion tables and Google Maps. Our long term goal is the development of a sustainable program for post-market surveillance of pharmaceuticals in Malawi.

\section{STUDENT PRESENTATIONS}

\section{S1 Factors associated with acceptability of vasectomy among married men in urban and rural Blantyre, Malawi: A case study of Chilomoni and Lirangwe}

Tchaiwe Zulu, Gilbert Nkadaseka Nthuluzi, Yamikani Idi Kalima

College of Medicine, University of Malawi, Blantyre, Malawi

\section{Introduction}

Vasectomy is one of the most effective contraceptive method that has been comparatively invisible. Prevalence of vasectomy in Malawi is $0.1 \%$ compared to bilateral tubal ligation which is $10.2 \%$. The aim of the study was to examine factors associated with acceptability of vasectomy among married men in urban and rural Blantyre, Malawi. The specific objectives of the research were; to assess perceptions about vasectomy among married men; to assess socio economic and cultural factors affecting vasectomy acceptance among married men and to assess health system factors that affect acceptance of vasectomy among married men. 


\section{Methods}

A total of 138 participants were included; 134 were married men; 4 were health workers and 2 focus group discussions of married women. A descriptive cross sectional study design was used for the study. Multi-stage sampling technique was used. Simple random sampling was used to select study areas. Two focus group discussions were conducted among married women using interview guides. Four health workers were interviewed from Chilomoni and Lirangwe Health Centres. The collected data was entered and analysed using Epi info version 7 . Thematic content analysis was used for the analysis of textual data.

\section{Results}

The majority of the participants $79.1 \%$ believed that vasectomy is beneficial and would encourage married men to undergo the vasectomy procedure. Most participants had positive perceptions towards vasectomy. Factors that were associated with low acceptability of vasectomy included; future uncertainty of need for children due to death of children or a wife; women are supposed to be on family planning; lack of information on vasectomy surgical procedures; vasectomy can tamper with sexual organ thereby reducing libido and fear that the community would mock vasectomised men. Health system factors included the unavailability of vasectomy services at Chilomoni and Lirangwe health centres, health workers were not trained to provide vasectomy procedures and lack of equipment for vasectomy surgical procedures

\section{Conclusions}

Expansion of male oriented family Planning services and education can help to boost family planning acceptability among men, including vasectomy. Education on family planning can enhance vasectomy knowledge level hence overcoming some myths and misconceptions of vasectomy.

\section{S2 Implementation of the 5S Model in Malawi: A case study of Mzimba South and Chiradzulu district hospitals}

\author{
Robert Saizi, Mesilina Kausiwa, Jamester Chilunjika \\ College of Medicine, University of Malawi, Blantyre, Malawi
}

\section{Introduction}

In an effort to improve quality of healthcare, Ministry of Health adopted 5S quality improvement model in 2007. The model uses five Japanese names which translate as sort, set, shine, standardize and sustain. It is a low cost approach aimed at managing work environment with an intention of improving efficiency, eliminating waste and increasing process consistency. Like many other countries that have implemented 5S model, evaluation of district hospitals in Malawi has shown great variations in success in its implementation. This is so despite that all implementing districts had undergone similar processes to prepare them to successfully implement the model.

\section{Methods}

A comparative study was done between Mzimba South and Chiradzulu district hospitals. Descriptive cross sectional design using qualitative methods was used to study the research question. Semi-structured interview guide was used to collect data. The data was transcribed from audio to text and analysed using manual thematic analysis. A purposeful sample of 15 participants was selected at each study site.

\section{Results}

The factors that affected successful implementation of $5 \mathrm{~S}$ were (1) top management support and leadership (2) commitment, (3) motivation, (4) team spirit, (5) supervision and (6) training.

\section{Conclusions}

The study findings demonstrate the need to channel more effort in addressing the factors that affected successful implementation of the model. As such, Ministry of Health and key stakeholders should critically look into the factors as the model is rolling out to many hospitals to improve implementation.

\section{S3 Barriers and enablers to implementing the "Reaching Every Child" approach for improving immunisation services in Malawi: Health care workers' perspective}

Atupele Kapito-Tembo ${ }^{1}$, Geofrey Chirwa ${ }^{2}$, Moussa Valle ${ }^{2}$, Chawanangwa Chirwa ${ }^{1}$, Thomas Makwinja ${ }^{1}$, Madalitso Zulu ${ }^{3}$, Waleke Khumalo ${ }^{3}$, Allan Macheso ${ }^{4}$, Don Mathanga ${ }^{1}$, Kyaw Aung ${ }^{4}$

1.Malaria Alert Centre, College of Medicine, University of Malawi, Blantyre, Malawi

2. Expanded Programme on Immunisation, Ministry of Health, Lilongwe, Malawi

3. College of Medicine, University of Malawi, Blantyre, Malawi

4. UNICEF Malawi, Lilongwe, Malawi

\section{Introduction}

WHO developed a "Reaching Every Child" (REC) strategy to improve immunisation coverage by reaching un-immunised children such as those living in hard to reach communities. Between 2006 and 2010, the Ministry of Health with support from UNICEF trained health workers (HWs) in REC strategy in all districts Malawi. However, REC is not fully implemented in health facilities. We asked HWs to provide insights as regards barriers and enablers to REC implementation.

\section{Methods}

HWs attending REC micro-planning sessions from 5 low performing districts (Kasungu, Nkhata-Bay, Chitipa, Mchinji and Mzimba South) in Expanded Programme on Immunisation (EPI) were administered a standardised pre-session form in 2015. At least one HW from each facility in the districts attended the sessions. Information collected on REC included trainings attended, experiences and factors affecting REC implementation.

\section{Results}

Of the total $149 \mathrm{HWs}, 47 \%$ indicated they were previously trained in REC approach, varying from $25 \%$ to $76 \%$ in the districts. Twenty percent $(20 \%)$ of the HWs mentioned they were ever oriented or involved in facility-level REC micro-planning for EPI, varying from $4 \%$ to $35 \%$ in the districts. HWs identified availability of resources (55\%), HW skills/capacity including micro-planning $(23 \%)$ and supportive supervision and planning $(22 \%)$ as factors that facilitate REC implementation. HWs mentioned that lack of these factors, in the same order, is hindering REC approach implementation in facilities. Resources cited included transport, fuel, gas/kerosene for refrigerators, data tools, and suitable mode of transportation for hard to reach areas among others.

\section{Conclusions}

Despite previous REC approach trainings, most HWs are either not trained or do not possess micro-planning skills to implement REC strategy effectively. Making available resources for EPI, building HWs' skills and capacity, planning at facility level and supportive supervision will facilitate full implementation of REC strategy and improve immunisation coverage in Malawi. 
S4 An exploration of factors contributing to increased occurrence of teenage pregnancies in Malawi: The case of Mulanje District

Arthur Phiri ${ }^{1}$, Adamson S. Muula ${ }^{2}$, Grace Mzunzu ${ }^{1}$ and Cornelius Kunkeyani $^{1}$

1. Department of Health Systems and Policy, School of Public Health and Family Medicine, College of Medicine, University of Malawi

2. Department of Public Health, School of Public Health and Family Medicine, College of Medicine, University of Malawi, Blantyre, Malawi

\section{Introduction}

Adolescent pregnancies are increasing in sub-Saharan Africa; $16 \%$ of all births in the region are from girls aged 15-19 years per year. Malawi is second amongst countries with high incidences of teenage pregnancies. Nation-wide surveys and other studies indicate variations across regions and among districts. We were not aware of any specific studies conducted in Mulanje District on factors contributing to increased occurrences of teenage pregnancies.

\section{Methods}

This was an exploratory qualitative study with purposive selection of key informants and focus group discussions (FGD) with community members. Multi-stage and convenient sampling techniques used to draw the sample of adolescents' participants. Qualitative data were collected using an interview guide approach with respondents within in-depth interviews with key informants and FGD. Thematic content analysis was done for qualitative data.

\section{Results}

We identified factors contributing to increased occurrences of teenage pregnancies, and these were: cultural practices; socioeconomic status; peer pressure; poor relationships between parents and adolescents; and gender inequality

\section{Conclusions}

Teenage pregnancy is an important issue in Mulanje district. There is an urgent need to adopt new approaches such as further research and multi-sectoral integration to address unplanned adolescent pregnancies with more efficient and effective interventions that improve young people's practices, beliefs, knowledge and attitudes towards family planning as well as increase their access and utilization of sexual reproductive health services and information.

\section{POSTERS}

\section{P001 Predictors of uptake and timeliness of newly introduced pneumococcal and rotavirus vaccines, and measles vaccine in rural Malawi: A population cohort study}

$\underline{\text { Hazzie Mvula }}^{1}$, E. Heinsbroek ${ }^{2}$, M. Chihana ${ }^{1}$, A.C. Crampin ${ }^{1,3}$, S. Kabuluzi ${ }^{4}$, G. Chirwa ${ }^{4}$, C. Mwansambo ${ }^{5}$, A. Costello ${ }^{6}$, N.A. Cunliffe $^{2}$, R.S. Heyderman ${ }^{7,8,9}$, N. French ${ }^{2,9}$, N. Bar-Zeev ${ }^{2,9}$, VacSurv Consortium

1. Karonga Prevention Study, Chilumba, Malawi.

2. Institute of Infectious and Global Health, University of Liverpool, Liverpool, United Kingdom

3. London School of Hygiene and Tropical Medicine, London, United Kingdom

4. Expanded Programme on Immunisation Office and Preventive Services Office, Ministry of Health, Lilongwe, Malawi

5. Health Services, Ministry of Health, Lilongwe, Malawi

6. Institute of Global Health, University College London, London, United Kingdom

7. Liverpool School of Tropical Medicine, Liverpool, United Kingdom 8. Division of Infection \& Immunity, University College London, London, United Kingdom

9. Malawi-Liverpool-Wellcome Trust Clinical Research Programme, Blantyre, Malawi

\section{Introduction}

Vaccination is the most strategic way of preventing morbidity and mortality from childhood diseases, but oftentimes there are selected groups that achieve poorer coverage than the national average. We analysed predictors of uptake and timeliness of newly introduced pneumococcal conjugate vaccine (PCV13) and rotavirus vaccine (RV1) and of measles vaccine (MV) in order to identify potential gaps in vaccination coverage and timeliness.

\section{Methods}

This was a four year prospective population based birth cohort study of 2, 616 children in the Karonga Health and Demographic Surveillance System (KHDSS), northern Malawi, from August 2011 to November 2015. All vaccination age-eligible children at the dates of introduction of PCV13 or RV1 were eligible for enrolment. Study staff visited households and collected data on individual and population level characteristics and transcribed child vaccination records from parent held child booklets on to study questionnaires.

\section{Results}

Vaccine coverage was high for all vaccines and raged from $86.9 \%$ for RV1 dose 2 to $95.4 \%$ for PCV13 dose 1 . Median delay for receiving vaccination was 17 days (IQR 7-36) for PCV13 dose 1, 19 days (IQR 8-36) for RV1 dose1 and 20 days (IQR 3-46) for MV. Lower maternal education, farming and far distance from road or clinic predicted being not fully vaccinated or late vaccination. Nonfacility birth was also associated with late vaccination.

\section{Conclusions}

Despite high vaccination coverage, delays in vaccination were common in this setting. We identified individual and programmatic predictors for uptake and timeliness of vaccination. Malawi's vaccine programme is very successful, but there is a need for vaccination delivery to also specifically target those at high risk of not being vaccinated fully or on time in order to maximise the benefit achieved by vaccination. 
P002 Assessment of nutrition therapy knowledge and practices among health care workers in a paediatric burns unit: A case of Kamuzu Central Hospital

\author{
Samuel Mwango, Getrude Mphwanthe \\ Lilongwe University of Agriculture and Natural Resources, Bunda \\ Campus, Lilongwe, Malawi
}

\section{Introduction}

Burns are the 6th leading cause of death among U-5-year-old children worldwide. Through Millennium Development Goal (MDG) 4 Malawi targets to reduce under five mortality by two thirds by 2015. The concept that nutritional support plays an indisputable role in treating critically ill patients is well-accepted by scientific and health professionals. However, nutrition knowledge and better practices are a preliquisite in ensuring proper nutrition for patients.

\section{Objectives}

To assess health workers' nutrition knowledge, practices and availability of protocols in burn management.

\section{Methods}

The study was conducted at Kamuzu Central Hospital in the Surgical Department. Purposive sampling was used select 32 health workers. Data was collected using structured questionnaire. Knowledge levels were determined using the Michigan State University diabetics' knowledge assessment scale. Ethical approval was sought at Kamuzu Central Hospital and verbal consent was obtained from the participants,

\section{Results}

About $78.1 \%$ of the respondents were knowledgeable about nutritional therapy in pediatric burn patients. Respondents $(56.3 \%)$ reported that they take weight of burn patients only on admission, whilst $37.5 \%$ and $3.1 \%$ reported that weight is taken on daily and weekly basis, respectively. When determining energy and nutrients need of the burn patients a recommended formula is used, however it was found out that $68.8 \%$ of the respondent they do not use it, implying that patient nutrition needs are not estimated. Respondents claimed that lack of expertise and resources contributed to poor practices. Availability and following standard nutrition protocol in case management is very important, however, $87.5 \%$ of the respondents reported that the burns unit had a standard nutrition therapy protocol but could not show it whilst $12.5 \%$ reported the unit had no protocol.

\section{Conclusions}

Respondents nutrition knowledge is not below par; however more has to be done on nutrition practice and resource availability in ensuring proper nutrition care for burn patients.

\section{P003 Repellence Effect of pyrethroids on mosquitoes}

\section{Elizabeth Bandason $^{1,2,3}$, Peng $\mathrm{Xu}^{1}$, Ke Dong ${ }^{1}$}

1. Michigan State University, East Lansing, Michigan, USA

2. Malaria Alert Centre, College of Medicine, University of Malawi, Blantyre, Malawi

3. Lilongwe University of Agriculture and Natural Resources, Bunda Campus, Lilongwe, Malawi

\section{Introduction}

For decades, Pyrethroid interaction with the sodium channels in the insects have been a widely accepted hypothesis. Contact or ingestion are well known routes of exposure to pyrethroids for insects. Recent studies reveal the insects' ability to be deterred or repelled from treated arenas through olfactory pathways without contact.

\section{Methods}

A laboratory raised strains of Aedes aegypti (Waco strain) and Peurto Rico strain were used in Arm in cage landing assay. Four to ten day old, starved and nulliparous mosquitoes were used. Fifty female mosquitoes were recruited for each experiment, replicated 5 times. Pyrethroid, serial dilutions of 10-2 to 10-8 were prepared and used in arm in cage landing assay. The insect repellent, DEET N,N-diethyl-3-methylbenzamide (a non-pyrethroid) was used as a positive control and blank solvent (acetone) was used as a negative control.

\section{Results}

A dose dependent landing response was evident in all the pyrethroids that were used in the assay. Different pyrethroids elicited different landing responses. Transfluthrin elicited higher repellence compared to permethrin and pyrethrum.

\section{Conclusions}

These preliminary findings present a most likely new mode of action of pyrethroids.

\section{P004 Recurrent malaria in a patient on ART illustrates the need for more evidence for management of a common medical challenge}

Wongani Nyangulu ${ }^{1}$, Edson Mwinjiwa ${ }^{1}$, Titus Divala ${ }^{2}$, Randy Mungwira $^{2}$, Osward Nyirenda ${ }^{2}$, Maxwell Kanjala ${ }^{2}$, Gillian Mbambo $^{3}$, Terrie E. Taylor ${ }^{2}$, Mathew B. Laurens ${ }^{3}$, Miriam K. Laufer ${ }^{3}$, Joep J. van Oosterhout ${ }^{1,4}$

1. Dignitas International, Zomba, Malawi

2. Blantyre Malaria Project, College of Medicine, University of Malawi, Blantyre, Malawi

3. Division of Malaria Research, Institute for Global Health, University of Maryland School of Medicine, Baltimore, Maryland, USA

4. Department of Medicine, College of Medicine, University of Malawi Blantyre, Malawi

\section{Introduction}

Co-treatment of HIV and malaria is common in Sub-Saharan Africa. Several factors can impact on malaria treatment outcomes in such patients. We describe a case illustrating complex issues that can lead to recurrent malaria, including clinically relevant drug interactions.

\section{Case presentation}

A 49-year-old woman on tenofovir/lamivudine/efavirenz (5A) with good response to ART (undetectable viral load; CD4= 461 cells $/ \mu \mathrm{L}$ ) and no recent history of malaria, stopped cotrimoxazole prophylaxis upon enrolment into a clinical trial. Four weeks later she presented with Plasmodium falciparum malaria diagnosed on blood film, was treated with artemether-lumefantrine (LA), and underwent formal malaria treatment evaluation according to WHO guidelines. After successful treatment assessed at 28 days, the patient experienced three subsequent malaria episodes over 4 months, one of which was due to $P$. ovale. All episodes were treated with LA and parasitemia resolved within 3 days. The last episode occurred 21 days after LA treatment initiation, raising suspicion about late parasitological failure. PCR testing confirmed the species identified by microscopy, with only one episode containing $P$. ovale infection.

\section{Discussion}

Drug interactions between lumefantrine and efavirenz, $P$ ovale hypnozoite relapse, possible suboptimal LA adherence and stopping 
cotrimoxazole prophylaxis may have contributed to recurrent malaria episodes in this patient. Drug interactions between efavirenz and lumefantrine, which normally has a long half-life of 3-6 days, contribute to enhanced metabolism of lumefantrine, and a shorter-than-expected prophylactic duration after successful treatment of an initial infection. In some individuals, efavirenz is metabolized slowly due to genetically-determined factors, and they maintain high efavirenz levels, leading to stronger CYP 3A4 enzyme induction and even more rapid lumefantrine metabolism.

\section{Conclusions}

There was no evidence of antimalarial drug resistance in this case but more research is needed to assess the public health importance of LA-efavirenz drug interactions.

\section{P005 Barriers and facilitators to accessing village clinic services for the improvement of maternal and child health services in rural Blantyre}

\author{
$\underline{\text { Daniel Mwale }}^{1}$, Lucinda Manda-Taylor ${ }^{1}$ \\ College of Medicine, University of Malawi, Blantyre, Malawi
}

\section{Introduction}

The World Health Organization (WHO) reports that every year about 12 million children die before their fifth birthday. Pneumonia, diarrhoea and malaria remain leading causes of death for under-five children and access to effective and appropriate treatment for sick children is extremely low where it is needed most. Therefore, community-based service delivery and interventions through trained community health workers (CHWs) have received renewed global interest as a strategy to expand access to effective interventions and treatment of childhood illnesses. This study uncovers obstacles and enabling factors underpinning utilization of village clinics (VCs) by mothers of under-five sick children in rural Blantyre.

\section{Methods}

This was a descriptive-qualitative study. Twenty-four in-depth interviews were conducted with mothers of under-five sick children accessing care at the facilities and those residing in the communities. Health Surveillance Assistants (HSAs) were also interviewed from each facility. Participants were purposively selected from eight randomly selected health facilities in rural Blantyre.

\section{Results}

Participants attributed VCs proximity and good quality of HSAs services as enabling factors. Proximity of VCs was related to easy access of services. High-quality HSAs service was accredited to proper assessment of sick children and Panadol or LA prescription. However, poor quality of VCs services, drug stock out, HSAs residence and HSAs workload impeded mothers' VC access.

\section{Conclusions}

Constant supply of essential drugs and permanent availability of HSAs at the VCs and within the community are fundamental for effective delivery of VCs services. However, various factors impede delivery. Stakeholders should have good understanding of these facilitators and barriers in order to improve delivery of VCs in the community.

\section{P006 Malaria control: A systematic review}

Tumaini Malenga, Lucinda Manda-Taylor, Francis Griffiths

College of Medicine, University of Malawi, Blantyre, Malawi

\section{Introduction}

In Sub Saharan Africa, malaria is more prevalent in rural areas with poor health systems. This has influenced the research into malaria control in terms of developing tools that can be easily adopted for such settings. Recent efforts for malaria eradication look towards a multifaceted approach to disease prevention. This paper presents a realist review on various malaria control strategies, to uncover what works or doesn't work, for whom and in what context. The paper works to inform what current malaria control strategies are most useful and what barriers exist with access and use, to discover the gaps in current knowledge.

\section{Methods}

A scoping review of literature on malaria control; a search for evidence of what works or doesn't work; extraction of data and synthesis of evidence; and development of a narrative which includes a hypothesis.

\section{Results}

Most behaviour change programs are based on socio-cognitive that aim to improve uptake and use of interventions, to adopt behaviours that improve health. The deficit in literature exists with theory promoting health behaviour change maintenance. There is a wealth of evidence that speaks to why malaria interventions don't work. In a situation where, if the intervention works, what guarantees or supports behaviour maintenance with malaria control interventions?

\section{Conclusions}

By understanding how the intervention works and seeing it work consistently over time, improves the buy in and likelihood of an individual to comply with use and maintain use in the long term. Assuming they accept the design and experience minimal barriers.

\section{P007 Exploring malaria parasite transmission dynamics following enhanced intervention around Majete Game Reserve using a population genetics approach}

\section{Lizzie Tchongwe-Divala $^{1}$, Alinune Kabaghe ${ }^{2}$, Robert S. McCann ${ }^{3}$, Michelle van Vugt ${ }^{4}$, Kamija Phiri ${ }^{2}$, Dianne J. Terlouw ${ }^{1,2,5}$, Standwell Nkhoma ${ }^{1,2,5}$}

1. Malawi-Liverpool-Welcome Trust Clinical Research Programme

2. College of Medicine, University of Malawi, Blantyre, Malawi

3. Wageningen University, Wageningen, the Netherlands

4. University of Amsterdam, Amsterdam, the Netherlands,

5. Liverpool School of Tropical Medicine, Liverpool, United Kingdom

\section{Introduction}

Accurate methods for monitoring the progress of malaria control efforts at subnational level are urgently needed to inform national control policies. Population genetic monitoring, a method that utilizes the relationship between malaria transmission intensity and parasite population structure, may provide a novel monitoring option. The higher the transmission intensity, the higher the genetic diversity, effective population size, multilocus linkage disequilibrium, and proportion of multiple-genotype infections. In this new study we aim to identify genetic parameters associated with transmission level changes based on data from rolling crosssectional surveys with known transmission variation during a period of scale of up control efforts in communities within the perimeter of the Majete Wildlife Reserve in Chikwawa District. 


\section{Methods}

We will genotype malaria parasite isolates sampled from selected intervention and control areas using 24-SNP barcode assay to identify the following parameters: multiple-genotype infections (MIs), mean number of heterozygous SNPs within MIs, parasite genetic diversity, multilocus linkage disequilibrium and effective population size $(\mathrm{Ne})$. Using multiple logistic regression analysis, we will determine the population genetic parameters that best predict malaria transmission and develop a malaria transmission prediction algorithm.

\section{Results}

This is an ongoing study, I propose to present the study design phase of the project, detailing study design challenges, choices and selection study methods to discuss these with senior academic seniors and peers.

\section{Conclusions}

Population genetic monitoring may prove an accurate method for tracking and predicting malaria transmission reduction. Our study will explore which parasite genetic parameters could be used for monitoring in Malawi.

\section{P008 Increased use of long-acting reversible contraception among Malawian women after implementation of a package of family planning interventions}

Clara Lemani ${ }^{1}$, James Chirombo ${ }^{1}$, Nenani Kamtuwanje ${ }^{1}$, Loyce Chikosi $^{4}$, Ann Phoya ${ }^{1}$, Timothy Bonyonga ${ }^{1}$, Mwawi Mwale ${ }^{5}$, Grace Chiudzu', Jennifer H. Tang ${ }^{1,2,3}$

1. UNC Project-Malawi. Lilongwe, Malawi

2. Department of Obstetrics \& Gynecology, University of North

Carolina, Chapel Hill, North Carolina, USA

3. Department of Obstetrics \& Gynaecology, College of Medicine,

University of Malawi, Blantyre, Malawi

4. Area 25 Health Centre, Lilongwe, Malawi

5. Lilongwe District Health Office, Lilongwe, Malawi

\section{Introduction}

Almost half of pregnancies in Malawi are unintended. One strategy to reduce unintended pregnancy is to expand modern contraceptive method mix and ensure that the most effective reversible contraceptives, long-acting reversible contraceptives (LARC), are available at health facilities. Our objective was to implement a package of family planning (FP) interventions at a health centre in Malawi to expand its modern contraceptive method mix and decrease reliance on short-acting reversible contraception (SARC).

\section{Methods}

We began implementing our FP interventions at Area 25 Health Centre in Lilongwe in February 2014. Interventions included: 1) Community sensitizations via Open Days and Population Weekends, 2) Provider trainings in LARC insertion, 3) FP mentoring, and 4) Weekly monitoring of family planning commodities. FP data were abstracted from facility's FP Reports and descriptive statistics were used to compare the proportion of SARC and LARC methods provided at the health centre one year pre-intervention and two one-year periods post-intervention.

\section{Results}

A total of $14,385,17,581$ and 17,430 women chose to receive a modern contraceptive method at Area 25 Health Centre during the pre- intervention, first year and second year post-intervention periods, respectively. During the pre-intervention period, $94.0 \%$ of all family planning clients chose SARC, and $4.9 \%$ chose LARC. During the first year post-intervention period, $87.7 \%$ of all family planning clients chose SARC, and $12.9 \%$.
Conclusions

FP interventions which focus on both increasing demand via community sensitization and supply via provider training and mentoring can increase utilization of LARC. However, further research should be done to examine barriers to IUD utilization, which still accounted for $<2 \%$ of modern contraceptive provision at the health centre.

P009 Experiences of caregivers of infants who have been on bubble continuous positive airway pressure at Queen Elizabeth Central Hospital, Malawi: A descriptive qualitative study

\section{Mtisunge Joshua Gondwe ${ }^{1}$, Belinda Gombachika ${ }^{2}$, Maureen Majamanda ${ }^{2}$}

1. Kamuzu College of Nursing, University of Malawi, Blantyre, Malawi 2. Department of Medical/Surgical Nursing, Kamuzu College of Nursing, University of Malawi, Blantyre, Malawi

\section{Introduction}

Bubble Continuous Positive Airway Pressure (BCPAP) is a form of oxygen treatment given to children with severe respiratory distress. The use of BCPAP is a new concept in Malawi and little is known about caregiver experiences on use of BCPAP on their infants. The purpose of this study was to explore the experiences of caregivers of infants who have been on BCPAP at Queen Elizabeth Central Hospital, Malawi

\section{Methods}

This was a qualitative descriptive study carried out at Chatinkha and Paediatric nursery wards at Queen Elizabeth Central Hospital. Purposive sampling technique was used to select caregivers of infants who had been on BCPAP. A sample size of 12 caregivers was decided upon after data saturation. Qualitative data was analysed using Colaizzi's framework

\section{Results}

Some caregivers were anxious, stressed, frightened, worried, disappointed and afraid of death while others were crying when they saw their infants on this treatment. Family members and friends were among preferred sources of psychological support compared to healthcare workers. The information given by health care workers about BCPAP was inadequate for caregivers to understand this treatment.

\section{Conclusions}

The results have shown that there are gaps in providing support to caregivers of infants on BCPAP at the hospital in terms of giving information and psychological care. As such, it is recommended that specific interventions be developed to minimize psychological distress and promote the psychological health of caregivers of sick infants on BCPAP at Queen Elizabeth Central Hospital.

\section{P010 Factors associated with uptake of intrauterine contraceptive devices and implants among women of childbearing age in Machinga District, Malawi: A qualitative exploratory study}

Mathews Chavunya, Adamson S. Muula

College of Medicine, University of Malawi, Blantyre, Malaw

\section{Introduction}

Uptake of Intrauterine Contraceptive Device (IUCD) and implants is very low in Machinga district despite some organizations 
including the Machinga District Health Office (DHO) addressing almost all of the factors identified by other studies as underlying the use of IUCD and implants in other areas. This comes against the backdrop of a high district fertility rate of 6.9 vs. 5.7 and 4.4 nationally in 2010 and 2015 respectively. Nationally, IUCD use still remained low at $1.1 \%$ in 2015 . The national data for IUCD and Implants use still indicate the need for more research into the factors associated with their utilization at district level including in Machinga.

\section{Methods}

Focus Group Discussions, In-depth interviews and Key Informant interviews were used to collect the data. A total of 120 (54 males, 66 females) participated in the study. Of these 10 (1 male, 9 females) were family planning providers and 10 ( 4 males, 6 females) were local leaders. The rest were community members. The participants were purposively selected. Data analysis was done through content analysis and theme generation.

\section{Results}

Among the many motivators and barriers identified, identified such factors as integration of services and un-intended consequences of other project interventions as barriers to LARC use. The factors were categorized under the individual, family and peer networks; community and system levels. The study also unveiled the risk of unintended consequences that economic empowerment programs may bring if they are not mainstreamed with family planning.

\section{Conclusions}

The findings highlight the importance of using the socioecological model in family planning programming so as to identify and address those factors at every level. The study exposes the weakness of implementing the socio-ecological approach with no regard for the systems thinking approach in implementing family planning programs.

\section{P011 Evidence of target-site resistance associated with pyrethroid use in Anopheles gambiae sensu lato: Majete case study}

Trancizeo Lipenga ${ }^{1}$, Robert S. McCann, ${ }^{2,3}$ Themba Mzilahowa ${ }^{4}$, Standwell Nkhoma ${ }^{1,2}$, Martin Donnelly ${ }^{5}$

1. Malawi-Liverpool-Wellcome Trust Clinical Research Programme, Blantyre, Malawi

2. College of Medicine, University of Malawi, Blantyre, Malawi

3. Wageningen University and Research, Wageningen, the Netherlands

4. Malaria Alert Centre, College of Medicine, University of Malawi,

Blantyre, Malawi

5. Vector Group, Liverpool School of Tropical Medicine, Liverpool,

United Kingdom

\section{Introduction}

Malaria vector control strategies largely rely on the use of long-lasting insecticides nets (LLINs) and/or indoor residual spraying. Scaling-up of these interventions has led to increasing selection pressure, with growing evidence of insecticide-resistant phenotypes in populations of all major malaria vector species. We determined phenotypic susceptibility levels of Anopheles gambiae s.l. populations in Chikwawa District to one insecticide used in LLINs. We also explored one potential molecular mechanism underlining resistance.

\section{Methods}

Adult Anopheles gambiae s.l. were raised from wild-caught larvae sampled from nine distinct aquatic habitats in the Majete area of Chikwawa. Un-fed adult mosquitoes were exposed to permethrinimpregnated papers according to WHO protocol. Specimens were further identified to species and screened for knock down resistance $(k d r)$ mutations using molecular markers.
Results

The highest observed mortality of An. gambiae s.l. exposed to permethrin was $75.3 \%(67 / 89)$ in specimens collected from Mwalija, with $100 \%(7 / 7)$ mortality in An. qaudrannulatus species and $73.1 \%$ (60/82) mortality in An. arabiensis species. Molecular genotyping revealed no record of $k d r$ mutation in neither $L 014 \mathrm{~S}$ nor $L 1014 \mathrm{~F}$ alleles.

\section{P012 Chloroquine-induced photosensitivity in a Malawian female on first line antiretroviral therapy}

Titus H. Divala ${ }^{1}$, Randy G. Mungwira ${ }^{1}$, Osward Nyirenda ${ }^{1}$, Lameck Khonde ${ }^{1}$, Joep J. van Oosterhout ${ }^{2,3}$, Miriam K. Laufer ${ }^{4}$, Matthew B. Laurens ${ }^{4}$

1. Blantyre Malaria Project, College of Medicine, University of Malawi, Blantyre, Malawi

2. Dignitas International, Zomba, Malawi

3. Department of Medicine, College of Medicine, University of Malawi, Blantyre, Malawi

4. Division of Malaria Research, Institute for Global Health, University of Maryland School of Medicine, Baltimore, Maryland, USA

\section{Introduction}

Dermatoses are common in people with HIV and etiology includes infections, malignancies and drugs. We present a case of photosensitive dermatosis in a participant of a clinical trial comparing chloroquine to cotrimoxazole prophylaxis with respect to improvement of morbidity and mortality in adults on ART. Chloroquine, an antimalarial drug discontinued in Malawi due to resistance, has recently generated research interest following return of efficacy. Chloroquine is known to cause pruritus, but photosensitivity is a little-known side effect. Both chloroquine and efavirenz can cause photosensitivity.

\section{Case presentation}

A 37-year-old Malawian woman with HIV presented with 3 weeks' history of progressive pruritic skin rash with discoloration on sunexposed areas of face, neck, upper chest and forearms. She denied recent fever or illness. She did not change cosmetic products and reported a balanced diet. At presentation, she was taking tenofovir/lamivudine/efavirenz and weekly chloroquine. Physical examination showed a micro-vesicular rash with dark grayish to bluish skin discoloration in sun exposed areas. Assessment of other systems was non-remarkable. Due to lack of alternate etiology, chloroquine induced photosensitivity was suspected.

\section{Investigations and management}

Her CD4 count, full blood count, and serum alanine aminotransferase and creatinine were all within normal limits and HIV viral load was undetectable. She was given oral antihistamine and topical steroid. Four weeks later, symptoms worsened and chloroquine was stopped. Pruritus resolved after four weeks, but hyperpigmentation took 25 weeks to resolve.

\section{Discussion and conclusions}

Diagnostic challenges exist when patients are taking medications with overlapping side effects. In this case, the temporal relationship of withdrawal of chloroquine to resolution of symptoms suggests chloroquine was the etiology. Long term chloroquine use may cause photosensitivity and skin hyperpigmentation due to its high affinity to melanin. For individuals on chloroquine, clinicians should prioritize careful surveillance of skin reactions and discontinue use when photosensitivity is diagnosed. 
P013 Malaria morbidity and mortality before and after first national universal bed net distribution campaign: A facility-based malaria surveillance study

$\underline{\text { Atupele Kapito-Tembo }}^{1}$, Clarissa Valim ${ }^{2}$, Andy Bauleni ${ }^{1}$, Osward Nyirenda ${ }^{3}$, Paul Pensulo ${ }^{3}$, Don Mathanga ${ }^{1,4}$, Terrie E. Taylor ${ }^{3,5}$, Miriam K. Laufer

1. Malaria Alert Centre, College of Medicine, Blantyre, Malawi

2. Department of Immunology and Infectious Diseases, Harvard School

of Public Health, Boston, Massachusetts, USA

3. Blantyre Malaria Project, College of Medicine, University of Malawi, Blantyre, Malawi

4. Department of Community Health, School of Public Health and Family Medicine, College of Medicine, Blantyre, Malawi

5. College of Osteopathic Medicine, Michigan State University, East Lansing, Michigan, USA

6. Division of Infectious Disease and Tropical Pediatrics, Center for Vaccine Development, University of Maryland School of Medicine, Baltimore, Maryland, USA

\section{Introduction}

Despite decades of social marketing and targeted insecticidetreated nets (ITN) distribution to under-five children and pregnant women, evidence suggests there is no/little decline in malaria morbidity in Malawi. In this facility-based study, malaria morbidity in patients was evaluated before-and-after first universal ITN campaign.

\section{Methods}

Malaria surveillance was established at Ndirande health centre and Queen Elizabeth central hospital in Blantyre, Thyolo and Chikhwawa district hospitals. Thyolo and Blantyre are in moderate while Chikhwawa is intense endemic area. Demographic and illness data were collected from 2012 to 2013 one week out of every four at each facility. All malaria suspected patients were tested for malaria. Comparison was for January-May 2012 and January-May 2013.

\section{Results}

Malaria positivity rates among 10,352 and 11,891 outpatients' malaria tested pre and post-campaign were $29 \%$ and $32 \%$; $\mathrm{p} \leq 0.0001$ respectively. The rate in outpatients increased from pre- to post-campaign in intense transmission (35\% vs. $48 \%$; $\mathrm{p} \leq 0.0001)$ compared to moderate transmission area $(24 \%$ vs. $20 \% ; \mathrm{p} \leq 0.0001)$ and in rural $(30 \%$ vs $36 \% ; \mathrm{p} \leq 0.0001)$ compared to urban $(24 \%$ vs $16 \% ; \mathrm{p} \leq 0.0001)$. There was no difference in pre- and post-campaign rates in children $<5$ years but a modest increase in children 5-15years and adults $>15$ years. Parasite density decreased from pre- to post-campaign among outpatients with positive results in all age groups. Among hospitalised patients with malaria, the proportion with severe malaria decreased from pre- to post-campaign $(67 \%$ vs. $46 \%$; $\leq 0.0001)$ and pattern was similar in all age groups. Mortality in hospitalised malaria patients was similar during pre- and post-campaign.

\section{Conclusions}

Following the first national universal ITN campaign, malaria morbidity did not decline especially in rural and high transmission areas but appeared to have reduced parasite density and severe disease in Malawi. Findings support increased universal ITN distribution in rural and high transmission areas and possibly consider adopting other control interventions to compliment ITNs in Malawi.
P014 Easy access sampling for malaria surveillance in Africa: A systematic review

\author{
S.S.S. Sesay ${ }^{1,2}, \underline{\text { D. Mategula }}^{1}$, E. Giorgi ${ }^{3,4}$, D.G. Lalloo ${ }^{2}$, P.J.
} Diggle $^{3,4}$, D.J. Terlouw ${ }^{1,2}$

1. Malawi-Liverpool-Wellcome Trust Clinical Research Programme, Blantyre, Malawi

2. Liverpool School of Tropical Medicine, Liverpool, United Kingdom 3. Lancaster Medical School, Lancaster University, Lancaster, United Kingdom

4. Institute of Infection and Global Health, University of Liverpool, Liverpool, United Kingdom

\section{Introduction}

Monitoring fine-scale heterogeneity of malaria burden could allow more targeted control efforts, but would require accurate and affordable survey tools. Sampling subgroups that are representative of the underlying population while they aggregate at easily accessible locations, so called "easy-access groups", may be an option. We reviewed the literature for EAG based survey approaches to monitor malaria burden, with a particular interest in studies which compared EAG based sampling with population sampling to estimate malaria burden and coverage.

\section{Methods}

We conducted a literature search for descriptive surveys and prospective studies involving any EAG study populations that determined malaria prevalence or other coverage or impact indicators. We conducted an additional search of surveys of easy access group based surveys compared with population based surveys to determine if EAG based estimates are representative of the underlying population based estimate.

\section{Results}

We will present search findings based on 19 reports of malaria surveillance sampling EAGs populations. We will discuss six reports that compared EAG-based estimates with contemporaneous population-based estimates, and use these to discuss the current evidence and research gaps around EAG based sampling.

\section{Conclusions}

EAG based sampling may provide a potential complementary cost-effective malaria survey tool at (sub) district level, but robust comparisons are needed to determine their relative accuracy and costs compared to gold standard household sampling.

\section{P015 Efficacy of Bacillus thuringiensis var israelinsis (Bti) on anopheline mosquito larvae in Zomba}

\section{Martin Chiumia}

Malaria Alert Centre, College of Medicine, University of Malawi, Blantyre, Malawi

\section{Introduction}

Malaria vector control is facing great challenges due to the emerging insecticide resistance. Therefore, there is need of novel strategies to overcome such challenges hence this study evaluated the efficacy of Bacillus thuringiensis var israelinsis (Bti) on anopheline mosquito larvae in Zomba. Two formulations of Bti were evaluated namely VectoBac ${ }^{\circledR} \mathrm{WG}$ (granular) and VectobaBac ${ }^{\circledR} 12 \mathrm{AS}$ (liquid) Bti.

\section{Methods}

Mosquito collection: Mosquito samples were collected using two methods; Adult wild caught blood-fed female mosquitoes and 
larvae from their breeding sites. Adult mosquitoes laid eggs and hatched into larvae, while those collected from the field as larvae were allowed to emerge into adults and got blood-fed with Rattus norvegicus Albinus, then laid eggs and hatched into larvae. Third in star larvae were used in all experiments.

Experimental set up: During this study, six different concentrations of Bti were set and 360 mosquito larvae were exposed to different concentrations and results were observed hourly for 10 hours, then after 24 hours and 48 hours. The experiment was replicated three times.

\section{Results}

The lower effective dosage of granular Bti to kill 100\% anopheles mosquito larvae after 48 hours of exposure was $103.41 \mathrm{~g} /$ ha and the $\mathrm{LT}_{50}$ and $\mathrm{LT}_{90}$ were $6.2 \mathrm{hrs}$ and $18.5 \mathrm{hrs}$ respectively. In addition, anopheles' mosquito larvae required $0.001 \mathrm{ml} / \mathrm{L}$ of liquid Bti to achieve $100 \%$ mortality within 48 hours of exposure.

\section{Conclusions}

Both liquid and granular Bti have shown to be effective against anopheline mosquito larvae, hence this has a direct impact in reducing adult mosquito populations and consequently a reduction in the transmission of malaria and other diseases that are spread by mosquito bites. This should be replicated in the actual field and on a larger scale since this was laboratory based.

\section{P016 Classroom and clinical competence of nurse tutors in Malawi nursing colleges}

\section{Noel Mbirimtengerenji ${ }^{1}$, F.M. Daniels ${ }^{2}$, P. Martin ${ }^{2}$}

1. Kamuzu College of Nursing, University of Malawi, Lilongwe, Malawi

2. University of the Western Cape, Cape Town, South Africa

\section{Introduction}

Nurses need to possess classroom and clinical teaching competences to perform the role of a tutor properly. There are 2 physicians and 38 nurses for every 100,000 people in Malawi. The staff shortage contributes to the difficulties nurses experience to conduct their work with maximum capabilities. The main objective of this research was to determine the classroom and clinical competencies of the nurse tutors in Malawi nursing colleges.

\section{Methods}

Exploratory, descriptive design which utilized both qualitative and sequential quantitative methods was applied to Iterated Purposive Probability Sampling (IPPS) of 8 nursing colleges in Malawi. The sample size of 129 students and 82 nurse tutors responded to two structured questionnaires whilst 40 in-depth interviews and 10 focus groups were conducted with 8 participants in each group. There were 40 variables for the teaching competences under five ranked Likert scale and the Cronbach`s Alpha was found to be 0.945 without standardisation and it was 0.912 after standardisation.

\section{Results}

In this study after doing a 40 competence self-assessment item analysis and student-tutor assessment, on the nurse tutors, it is clear that there is reduced level of nurse tutor achievement in most competences in nursing education both in classroom and at the clinical area. Nurse tutors even fail to ask proper questions during teaching (OR $\leq 0.385 ; 95 \% \mathrm{CI}(0.133 \pm 1.118) ; \mathrm{p} \geq 0.079)$. Despite the experience of the nurse tutors in teaching students for a long time there is still very poor competence of punctuality $(\mathrm{OR} \leq 0$. $912 ; 95 \% \mathrm{CI}(0.372 \pm 2.234) ; \mathrm{p} \geq 0.840)$. Nurse tutors do not manage in mobilising the teaching and learning resources for the students in Malawi nursing colleges (OR $\leq 0.340 ; 95 \% \mathrm{CI}(0.102 \pm 1.136)$ $; \mathrm{p} \geq 0.080)$.

\section{Conclusions}

As the provision of nursing care becomes more modernized and diverse, it is necessary for nurses to build competencies and skills after a thorough professional training to deliver effective teaching both in class and at the clinical area.

\section{P017 Building capacity to graduate the next generation of healthcare providers and educators in sub-Saharan Africa}

\section{B. Malewezi ${ }^{1}$, L. Cunningham ${ }^{1}$, T. Nyika ${ }^{2}$, S. Sayeed ${ }^{1}$, E. Stuart-} Shor ${ }^{1}$, V. Kerry ${ }^{1}$

1. Seed Global Health, Boston, Massachusetts, USA

2. Peace Corps, Malawi

\section{Introduction}

Resource-constrained countries face the double burden of high morbidity/mortality and limited human resources for health $(\mathrm{HRH})$. The critical shortage of HRH in 83 countries negatively impacts the provision of care and continued education of HRH. To mitigate this urgent problem, the Global Health Service Partnership (GHSP; Seed Global Health/Peace Corps) partners with nursing and medical schools in Sub-Saharan Africa (SSA), with a multi-year commitment, to place US advanced practice nurses and physicians for 1 year and pair them with a faculty counterpart.

\section{Methods}

Qualitative interviews (180 individual/group interviews; GHSP volunteers, $n=61$, SSA students, $n=234$; faculty, $n=110$ ) and data tracking were used to assess outputs and reported impact.

\section{Results}

Between 2013-2015, GHSP placed 69 educators in 13 medical and nursing schools in Malawi, Tanzania, and Uganda for one-year assignments. On average, annual output of each volunteer cohort included: 37,500 service-hours (clinical/classroom) provided; 100 courses/trainings taught; 2,500 faculty, staff, post-graduate, $\mathrm{MD} / \mathrm{RN}$ student trainees; and introduction of new educational initiatives (morning reports, bedside ultrasound training and skills lab instruction). Qualitative data suggest that enhanced (quantity/breadth) clinical supervision improved student clinical skills, confidence and ability to connect theory to practice. Faculty reported that enhancements in curricula pedagogy resulted in improved student critical thinking skills and the promotion of an open, collegial working environment.

\section{Conclusions}

Faculty shortages remain a chronic barrier to essential workforce expansion in resource-limited regions of the world. GHSP is an innovative partnership between PC, Seed, and African academic institutions that aims to improve medical/nursing education in SSA by building human resources for health. Quantitative and qualitative data from the first two years of the program demonstrate a positive impact. Future work will include expansion of the GHSP model to Swaziland and Liberia and continued monitoring of the program's impact on provider shortages and retention in GHSP countries. 
P018 Delayed project initiation not always a curse: Lessons learned from an HIV clinical trial in Malawi

Linda Chauma Mussa ${ }^{1}$, Kenneth Phiri ${ }^{1}$, Wongani Nyangulu ${ }^{1}$, Edson Mwinjiwa ${ }^{1}$, Josh Berman ${ }^{1}$, Titus Divala ${ }^{2}$, Randy Mungwira $^{2}$, Osward Nyirenda ${ }^{2}$, Miriam Laufer ${ }^{3}$, Matthew Laurens ${ }^{3}$, Joep J. van Oosterhout ${ }^{1}$

1. Dignitas International, Zomba Malawi,

2. Blantyre Malaria Project, College of Medicine, University of Malawi,

Blantyre, Malawi

3. University of Maryland School of Medicine, Baltimore, Maryland, USA

\section{Introduction}

In resource-limited settings, it is not unusual to experience delayed project start-up. Initiating projects later than originally planned may lead to implementing activities hastily in order to get back on schedule thereby compromising deliverables. However, recent experience from the initiation of an HIV clinical trial through Dignitas International (DI), a medical and research organization operating in Malawi showed that delayed project initiation is not always detrimental to its deliverables; rather, it can bring unexpected benefits.

\section{Methods}

In 2015, in partnership with the University of Maryland and the Blantyre Malaria Project (Malawi), DI initiated a National Institutes of Health (USA) funded HIV clinical trial. Project initiation was however postponed due to a global shortage of a study drug. The drug supplier estimated a 2-3 months delay for the study drug delivery.

Given this information, DI management evaluated the administrative implications. Rather than temporarily lay off the newly hired study staff, DI opted to utilize the delay to carryout enhanced training. Study protocol, SOPs and data collection tools were repeatedly reviewed and trained in. Twenty complete "dryruns" were carried out while only five were planned. Additional training visits were made to a nearby longer existing trial site to enhance training, confidence and familiarity with study procedures. During the initial study monitoring visit a considerably lower number of citations was reported than found on average in other sites. This performance was well above adequate and unexpected for a newly initiated site.

\section{Results}

Time gained due to delayed project initiation can be put into good use and yield positive results. In this case the "unplanned" training helped the study team to be well conversant with the study procedures.

\section{Conclusions}

When project initiation is delayed, research managers should balance short and medium-term considerations and consider the possible benefits of the delay.
P019 Barriers and enablers to implementing the "Reaching Every Child" approach for improving immunisation services in Malawi: Health care workers' perspective

Atupele Kapito-Tembo ${ }^{1}$, Geofrey Chirwa ${ }^{2}$, Moussa Valle ${ }^{2}$, Chawanangwa Chirwa ${ }^{1}$, Thomas Makwinja ${ }^{1}$, Madalitso Zulu ${ }^{3}$, Waleke Khumalo ${ }^{3}$, Allan Macheso ${ }^{4}$, Don Mathanga ${ }^{1}$, Kyaw Aung ${ }^{4}$

1.Malaria Alert Centre, College of Medicine, University of Malawi, Blantyre, Malawi

2. Expanded Programme on Immunisation, Ministry of Health, Lilongwe, Malawi

3. College of Medicine, University of Malawi, Blantyre, Malawi

4. UNICEF Malawi, Lilongwe, Malawi

\section{Introduction}

WHO developed a "Reaching Every Child" (REC) strategy to improve immunisation coverage by reaching un-immunised children such as those living in hard to reach communities. Between 2006 and 2010, the Ministry of Health with support from UNICEF trained health workers (HWs) in REC strategy in all districts Malawi. However, REC is not fully implemented in health facilities. We asked HWs to provide insights as regards barriers and enablers to REC implementation.

\section{Methods}

HWs attending REC micro-planning sessions from 5 low performing districts (Kasungu, Nkhata-Bay, Chitipa, Mchinji and Mzimba South) in Expanded Program on Immunisation (EPI) were administered a standardised pre-session form in 2015. At least one HW from each facility in the districts attended the sessions. Information collected on REC included trainings attended, experiences and factors affecting REC implementation.

\section{Results}

Of the total $149 \mathrm{HWs}, 47 \%$ indicated they were previously trained in REC approach, varying from $25 \%$ to $76 \%$ in the districts. Twenty percent $(20 \%)$ of the HWs mentioned they were ever oriented or involved in facility-level REC micro-planning for EPI, varying from $4 \%$ to $35 \%$ in the districts. HWs identified availability of resources (55\%), HW skills/capacity including micro-planning $(23 \%)$ and supportive supervision and planning (22\%) as factors that facilitate REC implementation.

HWs mentioned that lack of these factors, in the same order, is hindering REC approach implementation in facilities. Resources cited included transport, fuel, gas/kerosene for refrigerators, data tools, and suitable mode of transportation for hard to reach areas among others.

\section{Conclusion}

Despite previous REC approach trainings, most HWs are either not trained or do not possess micro-planning skills to implement REC strategy effectively. Making available resources for EPI, building HWs' skills and capacity, planning at facility level and supportive supervision will facilitate full implementation of REC strategy and improve immunisation coverage in Malawi. 


\section{P020 Supporting family medicine development through academic partnership}

\author{
A. McDonald, E.C. Hutchinson, M.T. Makwero, A. Otubu, J. \\ Cunningham, B. Malewezi \\ 1. School of Public Health and Family Medicine, College of Medicine, \\ University of Malawi, Blantyre, Malawi \\ 2. WWAMI Network, University of Washington School of Medicine, \\ Seattle, Washington, USA \\ 3. Global Health Service Partnership/Seed Global Health, Boston, \\ Massachusetts, USA
}

\section{Introduction}

The Malawi Ministry of Health recognizes the value of Family Physicians to serve as leaders of primary health care teams in the district hospital setting. The COM School of Public Health \& Family Medicine (SPHFM) is committed to training Family Medicine (FM) registrars in the district setting where they will have their careers. Mangochi District Hospital (MDH) was chosen to be the first FM post-graduate training site. The University of Washington School of Medicine and associated residency programs, (WWAMI) have conducted decentralized FM training for over 4 decades. In order to support decentralized training in Malawi, a partnership between the SPHFM and WWAMI was established.

\section{Methods}

In 2015, 2 WWAMI FM residents at a time worked for one month alongside Malawian trainees at $\mathrm{MDH}$ (medical and $\mathrm{CO}$ students, registrars), to build collegial interactions, teach, and offer mentorship. At the end of year one, anonymous surveys were given to faculty, registrars, students (medical and CO), hospital staff (RN \& CO), and Health Surveillance Assistants at MDH. The surveys included questions assessing the impact of the US residents on the training experience, clinical care, morale, and specific learning objectives, as well as how to improve the efficacy of the partnership.

\section{Results}

Over 30 surveys were collected covering three cohorts of US residents. Based on a 5 -point Likert scale $(1=$ very negative to $5=$ very positive) the average responses were consistently between 3.5-5. Key findings include: impact on teaching, supervision and mentorship: 4.0; impact on clinical care: 4.68; impact on morale and understanding of Family Medicine principles: 4.0.

\section{Conclusions}

By partnering with an established FM program, the SPHFM and $\mathrm{MDH}$ were able to increase access to mentoring and supervision for trainees, strengthen the learning environment at $\mathrm{MDH}$, and demonstrate a positive impact on training and clinical care.

\section{P021 Impact of the PACAM STEP UP project on small finance contribution to 13 district hospitals in the Southern Region of Malawi}

\author{
Lameck Thambo, Fred Chiputula, Ida Lajabu \\ Palliative Care Association of Malawi, Lilongwe, Malawi
}

\section{Introduction}

Lack of space to provide palliative care services to patients is an issue in most Government public district hospitals in Malawi as a result palliative care services are offered in combination with other related services in a shared room in alternate days. Following this Palliative care services are offered once a week. This prevents many palliative care needy patients access to palliative care services due to congestion.

\section{Methods}

Palliative Care Association of Malawi (PACAM) through STEP UP project with funding from True Colours Trust did situation analysis in 13 district hospitals in southern region of Malawi to find out challenges hospitals are facing which retard palliative care service provision. Lack of space was a major problem identified amongst others. A memorandum of understanding with the hospital management was set on cost sharing and contribution for the refurbishment of palliative care clinic room. PACAM STEP UP project contributed finance support of MK 450.000 (\$600) to all 13 districts hospitals

\section{Results}

All 13 Public district hospitals who received finance support have an independent palliative care clinic room. 5 district hospitals are operating daily full time, 6 are operating twice a week and 2 are operating once a week. Palliative care services delivery and patients access to palliative care services have been increased in 13 hospitals. PACAM finance officer did financial audit in all 13 districts hospitals and no report of misuse of funds was found.

\section{Conclusions}

Finance support among others are the needs of hospitals to increase palliative care coverage. Investing Government public hospitals with small finance support by establishing MOU can increase palliative care service delivery and patients access to palliative care. When donors cannot provide direct funding to Government health facilities it can be channel through relevant NGO which complement the Government services in order to increase palliative care coverage.

\section{P022 Strengthening use of evidence in science communication: Activities at the Malawi- Liverpool-Wellcome Trust Clinical Research Programme}

Kate Gooding, Regina Makwinja, Rodrick Sambakunsi, Deborah Nyirenda, Nicola Desmond

Malawi-Liverpool-Wellcome Trust Clinical Research Programme, Blantyre, Malawi

\section{Introduction}

Engagement with research participants and wider society is a key part of research excellence and an important function of health research systems. The Malawi-Liverpool-Wellcome Trust Clinical Research Programme (MLW) undertakes public engagement to share findings and increase awareness of research, and community engagement to ensure research is ethical and locally appropriate. Activities include radio programmes, working with journalists, public exhibitions, outreach to students, science cafes, and community advisory groups.

MLW is working to strengthen use of evidence in public and community engagement through various research, monitoring and evaluation initiatives. This poster introduces some of these initiatives.

\section{Methods}

Various approaches and initiatives are being used to assess current public and community engagement, and to identify challenges and areas for future work:

- Developing theories of change as a basis for monitoring and evaluating Science Communication activities

- Collaboration with the Global Health Bioethics Network to conduct research and learn from research in other countries

- Research projects on ethics and engagement 


\section{Results}

So far these initiatives have identified several challenges and lessons about public and community engagement, for example difficulties facing fieldworkers and conditions affecting the value of Community Advisory Groups. These lessons are being used to revise and improve Science Communication activities.

\section{Conclusions}

Use of evidence to understand the effectiveness of public and community engagement activities is essential to avoid harm and maximize the benefits of MLW research. We hope our poster can contribute to exchange of lessons on Science Communication between research organizations in Malawi.

\section{P023 Implementation of the 5S Model in Malawi: A case study of Mzimba South and Chiradzulu district hospitals}

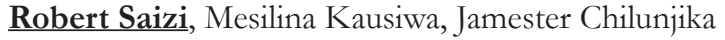 \\ College of Medicine, University of Malawi, Blantyre, Malawi
}

\section{Introduction}

In an effort to improve quality of healthcare, Ministry of Health adopted 5S quality improvement model in 2007. The model uses five Japanese names which translate as sort, set, shine, standardize and sustain. It is a low cost approach aimed at managing work environment with an intention of improving efficiency, eliminating waste and increasing process consistency. Like many other countries that have implemented $5 \mathrm{~S}$ model, evaluation of district hospitals in Malawi has shown great variations in success in its implementation. This is so despite that all implementing districts had undergone similar processes to prepare them to successfully implement the model.

\section{Methods}

A comparative study was done between Mzimba South and Chiradzulu district hospitals. Descriptive cross sectional design using qualitative methods was used to study the research question. Semi-structured interview guide was used to collect data. The data was transcribed from audio to text and analysed using manual thematic analysis. A purposeful sample of 15 participants was selected at each study site.

\section{Results}

The factors that affected successful implementation of $5 \mathrm{~S}$ were (1) top management support and leadership (2) commitment, (3) motivation, (4) team spirit, (5) supervision and (6) training.

\section{Conclusions}

The study findings demonstrate the need to channel more effort in addressing the factors that affected successful implementation of the model. As such, Ministry of Health and key stakeholders should critically look into the factors as the model is rolling out to many hospitals to improve implementation.
P024 Strengthening a network for evidenceinformed decision-making in health policy and practice in Malawi (EvIDeNt)

Collins Mitambo ${ }^{1}$, Dianne J. Terlouw ${ }^{2}$, Josh Berman ${ }^{3}$, Bertie Squire $^{4}$, Damson Kathyola ${ }^{5}$, Nyanyiwe Mbeye $^{6}$, Kamija Phiri ${ }^{6}$

1. Malawi Knowledge Translation Platform, Ministry of Health, Lilongwe, Malawi

2. Malawi-Liverpool-Wellcome Trust Clinical Research Programme, Blantyre, Malawi

3. Dignitas International, Zomba, Malawi

4. Collaboration for Applied Health Research \& Delivery, Liverpool

School of Tropical Medicine, Liverpool, United Kingdom

5. Department of Research, Ministry of Health, Lilongwe, Malawi 6. College of Medicine, University of Malawi, Blantyre, Malawi

\section{Introduction}

In Malawi's highly resource-constrained health sector, it is vital that decisions about allocation and prioritisation of public funds are taken on the basis of robust research and economic evidence that has been objectively synthesised and transparently and ethically considered.

\section{Methods}

A national case study of a network in development which aims to:

- strengthen cross-linkage and co-ordination between existing initiatives in the formulation and implementation of evidence-informed health decisions about policy and practice

- address identified gaps in local capacity to undertake economic evaluation and modelling and evidence synthesis

- $\quad$ address key hurdles in effective formulation of evidence informed health decisions

Over a 10-year period, we plan to build on existing research excellence to develop excellence in research uptake, decisionmaking and implementation.

\section{Results}

We will present a conceptual framework for the inter-linked steps that make up the process of evidence-informed decision making. We describe four recent key developments within Malawi that demonstrate the existence of developing expertise and leadership across the spectrum of evidence-informed health policy and practice. We lay out a 5-year strategic plan with seven objectives for the proposed network with key activities and lead partners for each.

\section{Conclusions}

The network will build on enabling developments within Malawi and support activities that are designed to strengthen existing expertise with support from established national and international academic groups and develop a diversity of funding streams in order to build resilience, responsiveness and sustainability. 
P025 Managing and monitoring chronic noncommunicable diseases in a primary health care clinic, Lilongwe, Malawi

Ronald Manjomo, Beatrice Mwagomba ${ }^{2}$, Serge Ade ${ }^{3,4}$, Engy Ali $^{5}$, Anne Ben-Smith ${ }^{6}$, Patricia Khomani ${ }^{1}$, Precious Bondwe ${ }^{1}$, Deliwe Nkhoma ${ }^{1}$, Gerald P. Douglas ${ }^{7}$, Katie Tayler-Smith ${ }^{5}$, Loyce Chikosi $^{8}$, Anthony D. Harries ${ }^{3,9}$, Oliver J. Gadabu ${ }^{1}$

1. Baobab Health Trust, Lilongwe, Malawi,

2. Department of Non-Communicable Diseases, Ministry of Health, Lilongwe, Malawi

3. International Union Against Tuberculosis and Lung Disease, Paris, France

4. National Tuberculosis Programme, Benin

5. Médecins sans Frontières, Medical Department, Operational Centre Brussels, MSF-Luxembourg, Luxembourg

6. Department of Biomedical Informatics, University of Pittsburgh,

Pittsburgh, Pennsylvania, USA

7. Center for Health Informatics for the Underserved, University of

Pittsburgh, Pittsburgh, Pennsylvania, USA

8. Area 25 Urban Health Centre, Lilongwe, Malawi

9. London School of Hygiene and Tropical Medicine, London, United Kingdom

\section{Introduction}

The burden of non-communicable diseases (NCDs) has been increasing in Malawi. As a policy response, the Ministry of Health established a unit within the ministry to coordinate the management and monitoring of NCDs in Malawi. Using an electronic medical record monitoring system, we carried out a study to describe the quarterly and cumulative disease burden, management and outcomes of patients registered between March 2014 and June 2015 at a primary health care facility.

\section{Methods}

This was a descriptive cross-sectional study involving patients registered with specific NCDs at Area 25 Health Centre, Lilongwe, Malawi.

\section{Results}

Of the 1135 patients registered, $66 \%$ were female, $21 \%$ were aged 65 years, $20 \%$ were obese, $53 \%$ had hypertension alone, $18 \%$ had diabetes alone, 12\% had asthma, 10\% had epilepsy and 7\% had both hypertension and diabetes. In every quarter, about $30 \%$ of patients did not attend the clinic and 19\% were registered as lost to follow-up (not seen for $>1$ year) in the last quarter. Of those attending, over $90 \%$ were prescribed medication, and 80-90\% with hypertension and/or diabetes had blood pressure/blood glucose measured. Over $85 \%$ of those with epilepsy had no seizures and $60-75 \%$ with asthma had no severe attacks. Control of blood pressure (41-51\%) and diabetes (15-38\%) was poor.

\section{Conclusions}

It is feasible to manage patients with non-communicable diseases in a primary health care setting in Malawi, although more attention is needed to improve clinic attendance and the control of hypertension and diabetes.
P026 Pregnancy intentions among HIV-infected women at 4-26 weeks post-partum in Option $\mathrm{B}+$ in Malawi

D. Thindwa $a^{5}$, M. Landes ${ }^{1,2}$, M. van Lettow ${ }^{1,3}$, A. Kanyemba ${ }^{5}$, E. $\mathrm{Nkhoma}^{5}$, H. Phiri ${ }^{5}$, J.J. van Oosterhout ${ }^{1,6}$, T. Kalua ${ }^{8}$, B. Tippett$\mathrm{Barr}^{7}$

1. Dignitas International, Zomba, Malawi

2. Department of Family and Community Medicine, University of Toronto, Toronto, Ontario, Canada

3. Dalla Lana School of Public Health, University of Toronto, Toronto, Ontario, Canada

4. Centers for Disease Control and Prevention, Lilongwe, Malawi

5. Management Sciences for Health, Lilongwe, Malawi

6. Department of Medicine, College of Medicine, University of Malawi,

Blantyre, Malawi

7. Centers for Disease Control and Prevention, Zimbabwe

8. Ministry of Health, Lilongwe, Malawi

\section{Introduction}

Avoiding unintended pregnancies is key to prevent pediatric HIV infection. We describe factors associated with pregnancy intentions among HIV-infected women presenting to under-5 clinics in Malawi.

\section{Methods}

A nested cross-sectional study was conducted in 2015 as part of a nationally representative cohort of HIV-infected women at 4-26 weeks postpartum in Malawi. Structured interviews collected data on socio-demographics, HIV/ART status and pregnancy intentions.

\section{Results}

414 HIV-infected women were enrolled; mean age was 28.1 years (95\%CI 27.5-28.7). Overall, 238/410 (58.0\%) women reported the index pregnancy was intended and $42.0 \%$ unintended. $223 / 411$ $(54.3 \%)$ and $150 / 411(36.5 \%)$ women had primary and secondary education respectively. A majority was Christian 356/412(86.5\%). Only $71 / 348(20.4 \%$ ) women had lost at least 1 child before this index pregnancy. Women over 30 years of age (OR 0.46, 95\%-CI $0.29-0.73, \mathrm{p}<0.001$ ) and those with $\geq 3$ children (OR: $0.36,95 \% \mathrm{CI}$ $0.20-0.66, \mathrm{p}<0.001)$ were less likely to have intended pregnancies. Known HIV status at conception $(n=374 / 408, p=0.251)$ was not significantly associated with pregnancy intention. However, $180(70.0 \%)$ women knew their partner was HIV-infected and were less likely to have intended pregnancy (OR 0.56, 95\%-CI $0.32-0.99, \mathrm{p}=0.044)$. A total of $333 / 389(85.6 \%)$ disclosed their status to their partners and this was not associated with pregnancy intention $(\mathrm{p}=0.841)$. In multivariable analysis, women with $<3$ children (aOR: 2.45, 95\%CI 1.30-4.64, $\mathrm{p}=0.006$ ) and those who started ART during (aOR: 1.76, 95\%CI 1.04-2.97, $\mathrm{p}=0.030$ ), rather than prior to index pregnancy were more likely to have intended pregnancy.

\section{Conclusions}

Rates of unintended pregnancies among HIV-infected women are high and associated with higher parity and age. This represents missed opportunity for PMTCT and family planning. Exploring how knowledge of HIV-infection for a woman and her partner, along with access to and uptake of ART, influences pregnancy intention could help inform family planning interventions in Option B+. 
P027 Facility-based strategies for enhancing retention and adherence among people with HIV and AIDS in Malawi: The expert client model of care

Alinafe Chibwana, G. Mateyu, V. van Schoor, I. Mayuni, Matengeni, J. Msonkho, Joep J. van Oosterhout, G. Sankhulani, F. Cataldo, D. Garone

Dignitas International, Zomba, Malawi

\section{Introduction}

In settings with high HIV burden and shortages of health staff, task-shifting strategies have used lay health workers to enhance retention in care and adherence to ART. Dignitas International and the Malawi Ministry of Health have scaled-up an Expert Client (EC) program in 106 health facilities in the South Eastern region of Malawi after a pilot phase at Zomba Central Hospital. ECs are HIV positive individuals who are open about their status and are trained in providing psychosocial support, health promotion and defaulter tracing. They work in ART-PMTCT and Under 5 clinics and receive remuneration as lay health workers.

\section{Methods}

Between August 2014 and December 2015, 328 ECs were enrolled. Routinely collected program data from 106 health facilities were used to explore the role of ECs in HIV service delivery.

\section{Results}

ECs reached 675,405 patients with health promotion messages, weighed 231,878 patients, and screened 56,069 children's nutritional status. $802 / 1,070(75 \%)$ defaulters identified by ECs returned into care, $98(9 \%)$ were not found, $107(10 \%)$ were on ART in other facilities and $63(6 \%)$ were deceased. ECs gave psychosocial support to 362,458 individuals of whom 47,997 were Option B+ women and followed-up 6,813 PMTCT women and 7,000 HIVexposed babies at their homes. Challenges in the program included dissatisfaction with low remuneration and continuing need for mentoring and training of an increasingly large workforce. ECs offered a remarkable range of support to patients and relieved the burden of formal health workers by performing health promotion, defaulter tracing and simple medical tasks.

\section{Conclusion}

Further evaluation of the contribution of ECs to reaching the 9090-90 UNAIDS targets is needed and how this compares to other types of lay-health workers, also in terms of cost-effectiveness. A more formal inclusion of ECs in the health care structure needs to be considered.

\section{P028 Factors associated with acquisition of HIV during 2005-2014 among men and women in 6 African cohorts}

\author{
E. Slaymaker ${ }^{1,7}$, M. Marston ${ }^{1,7}$, C. Calvert ${ }^{1,7}$ A. Dube ${ }^{2,3,7}$, \\ E. McLean ${ }^{1,3,7}$, C. Kanjala ${ }^{1,2,7}$, E. Martin ${ }^{1}$, D. Michaels ${ }^{1,7}$, A. \\ Takaruza $^{5,7}$, J. Nakiyingi-Miiro ${ }^{4}$, D. Nabukalu' ${ }^{7}$, T. Lutalo ${ }^{7}, \mathrm{~N}$. \\ $\mathrm{McGrath}^{7}$, D. Kwaro ${ }^{7}$, E. Geubbels ${ }^{6,7}$, B. Zaba ${ }^{1,7}$
}

1. Faculty of Epidemiology and Population Health, London School of Hygiene \& Tropical Medicine, London, United Kingdom

2. Malawi Epidemiology and Intervention Research Unit, Chilumba, Malawi

3. Department of Public Health, College of Medicine, University of Malawi, Blantyre, Malawi

4. Medical Research Council, Entebbe, Uganda

http://dx.doi.org/10.4314/mmj.v28i4.9
5. Biomedical Research and Training Institute, Harare, Zimbabwe

6. Ifakala Health Institute, Tanzania

7. ALPHA Network, London School of Hygiene \& Tropical Medicine,

London, United Kindgom

\section{Introduction}

As HIV/AIDS epidemics mature, treatment becomes widespread, and behaviour changes, correlates of incident HIV infection may change. We examined factors associated with HIV incidence during 2005-2014 among men and women aged 15 to 49 in order to implement targeted interventions

\section{Methods}

We analysed longitudinal data from six population-based cohorts from Kenya, Malawi, South Africa, Tanzania, Uganda and Zimbabwe with regular HIV testing to ascertain social and demographic factors associated with incident infection subsequent to ART rollout (2005-2014). We fitted piecewise exponential models to survival time to estimate hazard ratios (HR) for HIV incidence. Analysis time begins at the first negative HIV test and ends at sero-conversion or is right censored by death, out migration or the end of data collection.

\section{Results}

We found that current marriage, compared to never married, is associated with elevated hazard for young men aged 15 to 24 years (HR of 1.94, $\mathrm{p}<0.05$ ), but a reduced hazard for the older women (Hazard ratio of $0.52, \mathrm{P}<0.05$ ). Having left a marriage was a risk for older men aged 25-49 year (HR of 1.86, $\mathrm{p}<0.05$ ) and young women (HR of 2.61, $\mathrm{p}<0.05$ ). For young men, having one partner was associated with the Hazard ratio of $2.48, \mathrm{p}<0.05$ compared to the Hazard ratio of $7.09, \mathrm{p}<0.001$ for young men with four plus partners. Having a new partner increased women's hazard of seroconversion $(1.53, \mathrm{P}<0.05)$.

\section{Conclusions}

For younger women, risk increases as time since first sex increases, could be due to male partner seroconverting or due to acquisition from older already infected male partner. Factors associated with incidence vary between men and women and by age calling for the need to design and implement targeted interventions.

\section{P029 Growth patterns of HIV exposed uninfected children: Cohort study on children born before and after introduction of the "Option $\mathrm{B}+"$ programme in Malawi}

Malango T. Msukwa ${ }^{1,2}$, Andreas D. Haas ${ }^{1}$, Lyson Tenthani ${ }^{1,3}$, Cristina Barroso Hofer ${ }^{1}$, Adrian Spoerri ${ }^{1}$, Frank Chimbwandira ${ }^{4}$, Joep J. van Oosterhout ${ }^{5,6}$, Olivia Keiser

1. Institute of Social \& Preventive Medicine, University of Bern, Bern, Switzerland

2. The Baobab Health Trust, Lilongwe, Malawi

3. International Training \& Education Center for Health Malawi,

Lilongwe, Malawi

4. Department of HIV and AIDS, Ministry of Health, Lilongwe, Malawi

5. Dignitas International, Zomba, Malawi

6. Department of Medicine, College of Medicine, University of Malawi, Blantyre, Malawi

\section{Introduction}

There is concern that in-utero ARV exposure may impair the development of HIV exposed uninfected children. We compared weight-for-age-z-scores of HIV uninfected children who were retained in care and exposed to either AZT or $\mathrm{d} 4 \mathrm{~T} / 3 \mathrm{TC} / \mathrm{NVP}$ in 
the pre-Option $\mathrm{B}+$ period or to $\mathrm{TDF} / 3 \mathrm{TC} / \mathrm{EFV}$ in the Option $\mathrm{B}+$ period.

\section{Methods}

This was a retrospective cohort analysis of routine data on HIV exposed-uninfected children born between October 2009 and December 2014 at 21 health facilitiesin Malawi. We included all HIV exposed children with known sex, baseline weight, with at least one follow-up weight measurement and one valid HIV test result. We excluded children with either a positive HIV PCR test result or a positive HIV antibody test that was taken at least 12 months after birth. We used linear mixed effects models to assess weight-for-age-z-scores until 24 months of age according to the child's birth period. We defined birth period as pre-Option B+ if born before September 2011 and Option B+ if born after May 2012.

\section{Results}

We included 6,566 children (median follow-up duration 14.2 months; IQR 7.9; 21.1), 68.7\% were born in the Option B+ period and $50.0 \%$ were male. There were no differences in birth weight between children born pre-Option $\mathrm{B}+$ and those born during the Option $\mathrm{B}+$ period $(\mathrm{p}=0.700)$. Children born during the Option $\mathrm{B}+$ period grew faster than those born pre-Option $\mathrm{B}+$ (adjusted

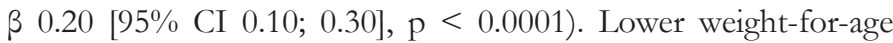
z-scores over time was independently associated with low birth weight, non-exposure to nevirapine prophylaxis at birth, nonexclusive breast feeding, any report of illness, and pre-Option $\mathrm{B}+$ birth period.

\section{Conclusions}

After the introduction of Option $\mathrm{B}+$ the growth rate of $\mathrm{HIV}$ exposed uninfected children improved, possibly due to effects related to improved health of the mothers.

\section{P030 Antiretroviral therapy and non- communicable diseases in HIV-infected patients in Malawi}

V. Tamba Tolno ${ }^{1}$, G. Guidotti ${ }^{2}$, H. Jere ${ }^{1}$, M. Giuliano ${ }^{3}$, J.B. Sagno $^{1}$, P. Germano ${ }^{2}$, G. Liotta ${ }^{4}$, D. Thole ${ }^{1}$, H. Sangarè ${ }^{1}$, M. Andreotti ${ }^{3}$, M. Floridia ${ }^{3}$, L. Palombi ${ }^{4}$, M.C. Marazzi ${ }^{5}$

1. DREAM Program, Blantyre, Malawi

2. DREAM Program, Rome, Italy

3. ISS, Rome, Italy

4. Biomedicine and Prevention Department, University of Rome, Rome, Italy

5. LUMSA University, Rome, Italy

\section{Introduction}

The burden of Non-Communicable Diseases (NCDs) is increasing in sub-Saharan Africa, and is becoming significant cause of morbidity and mortality. The objective of this study was to assess the prevalence of hypertension, raised blood glucose and obesity in a population of $\mathrm{HIV}$-positive patients naïve to antiretrovirals and to assess the impact of 6 years of ART on these parameters. selecting patients for 2 nd line ART.

\section{Methods}

A retrospective analysis was performed of routinely collected data of HIV-positive patients starting antiretroviral treatment in Malawi within the DREAM Program from January 2006 to May 2015. Multiple logistic regression models were used to identify factors associated with NCDs and the Cox model was used to assess predictors of survival in the cohort. All patients have been started on ARV with Stavudine/Lamivudine/Nevirapine that was the standard first line at that time in Malawi.

\section{Results}

A total of 6297 patients, mean age 37 years, females $57.4 \%$, were studied. At baseline the prevalence of hypertension was $10 \%$ (grade II hypertension was $5.2 \%$ ), diabetes was present in $0.3 \%$, obesity in $1.8 \%$ of the subjects. Increasing age, male sex and higher BMI were correlated to both hypertension and diabetes at baseline. After 6 years of ART on average the prevalence of hypertension was unchanged, diabetes and obesity slightly increased $(1.5 \%$ and $3.6 \%$ respectively). Male sex $(\mathrm{P}=0.017)$, increasing age $(\mathrm{P}<$ 0.001) low baseline BMI $(\mathrm{P}<0.001)$, low baseline hemoglobin $(\mathrm{P}<$ $0.001)$ and the presence of diabetes at baseline $(\mathrm{P}=0.001)$ were all independent predictors of mortality in the cohort.

\section{Conclusions}

Diabetes and obesity were rare in this HIV-infected population, while the prevalence of hypertension was moderate. Antiretroviral therapy had a modest impact on these conditions. These findings are re-assuring in a perspective of general progressive expansion of ART to all HIV-infected people.

\section{P031 Assessing implementation of models of differentiated care for HIV service delivery in Malawi: Multi-month prescriptions, fast- track refills, and community ART groups}

\section{Banda ${ }^{1}$, M. Prust ${ }^{1}$, F. Chimbwandira ${ }^{2}$, M. Eliya ${ }^{2}$, K. Callahan ${ }^{1}$,} M. Prescott ${ }^{1}$, E. McCarthy ${ }^{1}$, E. Tagar ${ }^{1}$, A. Gunda ${ }^{1}$

1. Clinton Health Access Initiative

2. Department of HIV and AIDS, Ministry of Health, Lilongwe, Malawi

\section{Introduction}

In Malawi, innovative models have been developed to optimize the efficiency of HIV service delivery in order to achieve the 90-90-90 targets. In particular, three key models of differentiated care have emerged: multi-month prescriptions; fast-track drug refills; and community ART groups. This study assessed the extent to which patients in Malawi are differentiated based on clinical stability and described the characteristics and costs of the models.

\section{Methods}

A mixed methods process evaluation was conducted in a sample of 30 purposefully selected ART facilities across Malawi. Qualitative methods included: interviews with 30 healthcare workers responsible for ART services; and 30 patient focus groups including 216 individuals. Quantitative methods included: clinical record reviews for 74,466 patients; orally-administered questionnaires with 136 health workers; observations of 714 patient visit times; and a questionnaire on facility characteristics in each of the 30 facilities.

\section{Results}

$47 \%$ of ART patients are eligible to be considered stable enough for differentiated care under $\mathrm{MOH}$ guidelines, but a limited number of eligible patients are receiving the models. For example only $39 \%$ of eligible patients are receiving multi-month prescriptions. Furthermore $38 \%$ of ineligible patients are receiving multi-month prescriptions. There are wide variations in the degree of patient differentiation across facilities. Patients and providers reported the benefits of differentiated models as reduced burden on patients 
and health workers and improved patient adherence. However, participants also highlighted challenges which among others included infrequent monitoring of patients which may cause adherence and other problems to go unnoticed for a longer period and challenges with supply chain support for the models.

\section{Conclusions}

There are major benefits to be offered by implementing differentiated models of care, but some challenges remain. In particular, adherence to guidelines for patient differentiation is poor and varied across facilities, and this must be improved to ensure high-quality care.

\section{P032 Expanding HIV and STI care to prisoners: The experience from Zomba Central Prison, Malawi}

D. Garone ${ }^{1}$, G. Mateyu ${ }^{1}$, M. Chigayo ${ }^{2}$, H. Ndindi', S. Gaven ${ }^{1}$, K. Harawa $^{1}$, V. Singano ${ }^{1}$, V. van Schoor ${ }^{1}$, Joep J. van Oosterhout ${ }^{1,3}$, A. Kwekwesa $^{1}$

1. Dignitas International, Zomba, Malawi

2. Malawi Prison Health Services

3. Department of Medicine, College of Medicine, University of Malawi, Blantyre, Malawi

\section{Introduction}

Globally, prevalence rates of HIV, sexually transmitted infections (STI's) and hepatitis B (HBV) in prison populations are 2 to 50 times higher than in general populations. Risks affect prisoners, prison staff, their families and the entire community. In 2013, Malawi Prison Services decided to scale-up HIV care in Malawian Prisons.

\section{Methods}

Since 2014 Dignitas International and the Malawi Prison Health Services have been implementing a comprehensive package of care and treatment for HIV-TB and STI's. Prisoners are routinely screened for HIV, TB and STI at entry and when they are released. In addition, HIV, TB, syphilis and HBV screening campaigns are conducted every six months. We used routinely collected program data to evaluate the HIV cascade and prevalence of syphilis and $\mathrm{HBV}$ in Zomba Central Prison.

\section{Results}

During a June 2015 screening campaign 1052/1745 (60\%) prisoners with unknown HIV status accepted testing for HIV, HBV and syphilis. 68/1052 (6.5\%) tested HIV positive, resulting in an overall prison HIV prevalence of 35\%. 52/1052 (4.9\%) tested positive for syphilis and 59/1052 (5.7\%) tested positive for HBV. $1.1 \%$ were co-infected with HIV/HBV. By October 2015, 482/539 (89\%) HIV positive patients were on ART, $52 \%$ were in WHO stages $3 / 4,48 \%$ had low CD4 $(<350$ or $<500$ cells $/ \mu \mathrm{L}$, depending on calendar episode). $98 \%$ were on the standardized $1^{\text {st }}$ line ART regimen. ART patients who were eligible for routine viral load (VL) monitoring according to National Guidelines received VL testing. $277 / 319(86 \%)$ were virologically suppressed $(<1000$ copies $/ \mathrm{ml}$ ).

\section{Conclusions}

Malawian Prisoners attained acceptable HIV testing coverage, high ART uptake, and good adherence demonstrated by high virological suppression. HIV care is feasible during incarceration for individuals that otherwise may be hard-to-reach. Prison health care programs need to address challenges such as confidentiality and continuity of care within and outside prisons.

http://dx.doi.org/10.4314/mmj.v28i4.9
P033 The burden of gynaecomastia among men on antiretroviral therapy in Zomba, Malawi

V. Singano $^{1}$, A. Amberbir ${ }^{1}$, D. Garone ${ }^{1}$, G. Mateyu ${ }^{1}$, J. Msonko, S. Chu' ${ }^{1}$, M. van Lettow ${ }^{1}$, K.Kalima ${ }^{1}$, J. Berman ${ }^{1}$, Y. Mataka ${ }^{1}$, A. Kwekwesa $^{1}$, A. Matengeni ${ }^{1}$, K. Phiri ${ }^{1}$, Joep J van Oosterhout ${ }^{1,2}$

1. Dignitas International, Zomba, Malawi

2. Department of Medicine, College of Medicine, University of Malawi, Blantyre, Malawi

\section{Introduction}

Gynaecomastia in HIV infected has been associated with hypogonadism and the use of several medications, most commonly efavirenz. After the introduction of the standardized first-line ART regimen $5 \mathrm{~A}$, which contains efavirenz, reports of gynecomastia appeared in popular media, however prevalence data of gynaecomastia in Malawi are lacking.

\section{Methods}

We conducted a cross-sectional study of adult males $(\geq 18$ years) on ART at the Tisungane HIV clinic, Zomba Central Hospital. Males were asked if they had ever experienced breast or nipple enlargement. Males who replied yes received a standard questionnaire for further details about gynecomastia and a physical examination. Clinicians confirmed the presence of gynecomastia along with grading according to accepted criteria. Patients reported perceptions and concerns about their gynecomastia. We extracted data on ART regimen, anthropometric measurements, history of tuberculosis (TB) and WHO clinical staging were extracted from males (irrespective of self-reported gynecomastia) from our electronic database.

\section{Results}

A total of 1,011 men were included in the study. The median age was 44 years (IQR: 38-52), median ART duration was 55 months (IQR: 26-83); 47\% were in WHO disease stage III/IV, 80\% had 5A (tenofovir/lamivudine/efavirenz) as current ART regimen; 9\% were overweight or obese. The prevalence of gynecomastia was 60/1011 (5.9 \%; $95 \%$-CI: $4.6 \%$ to $7.6 \%$ ) of whom $86 \%$ reported nipple enlargement (75\% bilateral) and $98 \%$ increased breast size $(85 \%$ bilateral), $44 \%$ had painful breasts, $3.4 \%$ had nipple discharge. $80 \%$ found the gynecomastia important or very important. Embarrassment was reported by $53 \%$, stigma by $10 \%$. On physical examination, $90 \%$ had confirmed gynecomastia; in $29(54 \%)$ this was grade 3/4. None had a testicular mass.

\section{Conclusions}

The burden of gynecomastia among males on ART in Malawi was higher than previously reported, and was associated with adverse psychological consequences, calling for increased awareness among patients and healthcare workers. 
P034 Contraceptive use and factors associated with unmet contraceptive need among HIVinfected women in Option B+ in Malawi

D. Thindwa ${ }^{5}, \underline{\text { M. Landes }}{ }^{1,2}$, M. van Lettow ${ }^{1,3}$, A. Kanyemba ${ }^{5}$, E. $\mathrm{Nkhoma}^{5}$, H. Phiri ${ }^{5}$, J.J. van Oosterhout ${ }^{1,6}$, T. Kalua ${ }^{8}$, B. TippettBarr $^{7}$

1. Dignitas International, Zomba, Malawi

2. Department of Family and Community Medicine, University of

Toronto, Toronto, Ontario, Canada

3. Dalla Lana School of Public Health, University of Toronto, Toronto,

Ontario, Canada

4. Centers for Disease Control and Prevention, Lilongwe, Malawi

5. Management Sciences for Health, Lilongwe, Malawi

6. Department of Medicine, College of Medicine, University of Malawi,

Blantyre, Malawi

7.Centers for Disease Control and Prevention, Zimbabwe

8. Ministry of Health, Lilongwe, Malawi

\section{Introduction}

Family planning and contraceptive use are cost-effective interventions for preventing HIV MTCT and maternal morbidity/ mortality from unintended pregnancy. We describe uptake of contraceptive methods and factors associated with unmet contraceptive needs among HIV-infected women 4-26 week postpartum in Option B+ in Malawi.

\section{Methods}

A nested cross-sectional study conducted in 2015 as part of a nationally representative cohort, collected data on sociodemographics, HIV/ART status, pregnancy intentions and contraceptives use through structured interviews.

\section{Results}

229/412(55.6\%) women used the following contraceptives: DepoProvera injection (67.2\%), In-plants $(12.7 \%)$, condoms $(10.5 \%)$, $\operatorname{oral}(6.6 \%), \operatorname{IUCD}(1.3 \%)$, and other(1.7\%). Of 172 women with unintended index pregnancy $105(61.1 \%)$ used contraception and $67(38.9 \%)$ had unmet need for contraception. Pregnancy intention was not significantly associated with contraception use $(p=0.072)$. Of 153 women with known HIV status at conception and who did not desire pregnancy, $60(39.2 \%)$ were not using contraception. Of 82 women who knew their partner was HIV-infected and did not desire pregnancy, 26(31.7\%) were not using contraception. Knowing one's own HIV status, knowing partner's HIV status and knowing partner was HIV infected were not significantly associated with contraceptive use ( $\mathrm{p}=0.061,0.400,0.696$ respectively). 335/389 $(86.1 \%)$ women disclosed status to their partners; non-disclosure was associated with lower contraceptive usage (OR $0.51,95 \% \mathrm{CI}$ : $0.29-0.92, \mathrm{p}=0.023)$. In univariable analysis, not using contraceptive among women with unintended pregnancy was associated with age $>20$ years (OR 0.46, 95\%-CI0.29-0.73, p<0.001), multiparity (OR 0.36, 95\%-CI0.20-0.66, $\mathrm{p}<0.001)$ and starting ART during index pregnancy (OR 0.41, 95\%CI-0.20-0.88, $\mathrm{p}<0.018$ ). In multivariable analysis, only multiparity remained independently associated (aOR: 0.41, 95\% CI: 0.22-0.77, $\mathrm{p}=0.006$ ).

\section{Conclusions}

Rates of unmet contraception need are high among women, including those who knew their HIV-status at time of conception and their partner's HIV-infected status. Unmet need for contraception was higher among women who are younger and with lower parity, representing a high risk groups for potential intervention.
P035 Impact of an adherence support program and genotypic resistance testing for second line ART eligibility

\author{
J.B. Sagno ${ }^{1}$, J. van Oosterhout ${ }^{2,3}$, G. Liotta ${ }^{4}$, R. Luhanga ${ }^{1}$, B. \\ Chilima $^{5}$, H. Jere ${ }^{1}$, L. Palombi ${ }^{4}$ \\ 1. DREAM Program, Blantyre, Malawi \\ 2. Dignitas International, Zomba, Malawi \\ 3. Department of Medicine, College of Medicine, University of Malawi, \\ Blantyre, Malawi; \\ 4. Biomedicine and Prevention Department, University of Rome 'Tor \\ Vergata', Rome, Italy; \\ 5. Ministry of Health, CHSU, Lilongwe, Malawi
}

\section{Introduction}

Of more than 500,000 Malawian patients on ART in 2015, around $98 \%$ are on first-line treatment. We aimed to evaluate an adherence support program carried out by the DREAM program in Malawi, and the potential role of HIVDR testing in selecting patients for 2nd line ART.

\section{Methods}

Among 2,614 patients on first-line ART in 5 DREAM health centers, routine VL monitoring after 12 months of ART was conducted between August 2011 and March 2013. Non-pregnant adults with VL $>1,000$ copies/ml underwent genotypic HIVDR testing. They received intensive adherence support for 6 months and at 18 months VL was repeated

\section{Results}

VL $>1,000$ at 12 months was found in $85(3.3 \%)$ patients. $65(76 \%)$ were female, mean age was 38 years. In multivariable analysis $\mathrm{VL}>1,000$ at 12 months was associated only with lower adherence. Genotyping showed $42(49 \%)$ patients harbouring major HIVDR mutations ( 8 to NRTI, 3 to NNRTI and 31 to both). Presence of HIVDR mutations was associated with lower BMI and CD4 count at ART initiation and with current ART regimen. After the adherence support program 41/82 (50\%) patients had VL $<1000$ copies/ml at 18 months, of whom 31 fully suppressed. 14/41 $(32.6 \%)$ with mutations at 12 months had $\mathrm{VL}<1,000$ at 18 months, this was $27 / 41(65.9 \%)$ for those without mutations $(p=0.012)$. Independent determinants of 18 months $\mathrm{VL}>1,000$ were higher $\mathrm{VL}$ at 12 months (OR 2.12; 95\%-CI: 1.02-4.47) and presence of HIVDR mutations at 12 months (OR 4.35; 95\%-CI: 01.59-11.1).

\section{Conclusions}

Similar to other experience, our adherence intervention for patients found with $\mathrm{VL}>1,000$ copies/ml upon routine VL monitoring at 12 months was highly effective in re-suppressing VL to below the 2nd line ART threshold. Presence of HIVDR mutations at 12 months was strongly associated with $\mathrm{VL}>1,000$ after adherence support, although an important percentage with mutations still suppressed. 
P036 The importance of cognitive interviews in the development of effective and efficient instruction for use tools: HIV self-testing Africa - Malawi

L. Kayira $^{1}$, M. Sambo ${ }^{1}$, W. Sibande ${ }^{1}$, E.L. Corbett ${ }^{1,2}$, N.

Desmond $^{1,3}$, M. Kumwenda ${ }^{1,4}$, W. Lora ${ }^{1,3}$

1. Malawi-Liverpool-Wellcome Trust Clinical Research Programme, Blantyre, Malawi

2. London School of Hygiene and Tropical Medicine, London, United Kingdom

3. Liverpool School of Tropical Medicine, Liverpool, United Kingdom

4. College of Medicine, University of Malawi, Blantyre, Malawi

\section{Introduction}

HIV self-testing (HIVST) is becoming an established option for providing highly accurate results when used by lay clients. To effectively use the HIVST kit (OraQuick), clients require to have a clear understanding and grasp of the instruction for use (IFUs) accompanying the test kit. Developing simplified and user-friendly is important to enable lay and illiterate people perform an HIV test without difficulty and promote the accuracy of interpretation of test results in absence of trained counsellors.

\section{Methods}

We conducted qualitative research using cognitive interviews (Cis) to improve IFUs for HIVST in urban and rural Blantyre, Malawi. 20 Cognitive interviews were carried out in four health centres during the first of the four iterative phases.

\section{Results}

Literate individuals in both urban and rural areas had ease with understanding, synthesising and translating pictorial and word instructions. Being able to grasp IFUs translated into easy performance of HIVST process and accurate interpretation of test results without support from a professional counsellor. Less literate individuals greatly dependent on pictorial instructions than word instruction to understand what they were required to do and had more errors in performing the HIVST.

\section{Conclusions}

Prior testing of IFUs for HIVST proved important in refining the instruction and fostering accurate interpretation of the instructions. Pictorial IFUs seemed more appropriate for a setting that has high levels of illiteracy.

\section{P037 Factors associated with lifetime experience of infant/child HIV-infection and mortality among HIV-infected mothers enrolled in Option B+ in Malawi}

\author{
M. Landes ${ }^{1,2}$, M. van Lettow ${ }^{1,3}$, A. Maida ${ }^{4}$, E. Schouten ${ }^{5}$, J.J. \\ van Oosterhout ${ }^{1,6}$, B. Tippett Barr ${ }^{7}$; on behalf of the NEMAPP \\ Consortium
}

1. Dignitas International, Zomba, Malawi

2. Department of Family and Community Medicine, University of

Toronto, Toronto, Ontario, Canada

3. Dalla Lana School of Public Health, University of Toronto, Toronto,

Ontario, Canada

4. Centers for Disease Control and Prevention, Lilongwe, Malawi

5. Management Sciences for Health, Lilongwe, Malaw

6. Department of Medicine, College of Medicine, University of Malawi,

Blantyre, Malawi

7. Centers for Disease Control and Prevention, Zimbabwe

http://dx.doi.org/10.4314/mmj.v28i4.9

\section{Introduction}

Cumulative child mortality is greater over an HIV-infected woman's lifetime than among uninfected women. We describe the infant/child mortality among HIV-infected women at 4-26 weeks postpartum within a national evaluation of Option $\mathrm{B}+$, and associations with fertility/PMTCT indicators.

\section{Methods}

A cross-sectional study of HIV-infected women at 4-26 weeks postpartum. Structured interviews collected data on sociodemographics, previous HIV-infected children/mortality and PMTCT indicators.

\section{Results}

$1140 \mathrm{HIV}$-infected women were enrolled. Total fertility rate was 7.7 births/woman and not significantly associated with timing of HIV diagnosis ( $\mathrm{p}=0.9)$. 965/1140(84.6\%) multiparous women reported 2400 previous live births; women diagnosed HIV+ prior to this pregnancy had more previous children ever tested than those diagnosed during this pregnancy $(\mathrm{p}=0.001)$. Timing of HIV diagnosis was not associated with having a previously diagnosed HIV-infected child $(p=0.475) .223 / 965(23.1 \%)$ multiparous women had $\geq 1$ previous child death (0-5 years) and $74(7.7 \%)$ had $>1$ previous child deaths. The infant mortality rate was 37.3 deaths $/ 1000$ births and the Under- 5 year mortality rate was 68.6 deaths/1000 births; both rates were greater among women diagnosed with HIV prior to- vs. during the index pregnancy $(67.0$ vs $29.3 ; \mathrm{p}=0.001$ and 96.0 vs $61.4 ; \mathrm{p}=0.001$ ).

In multivariable analysis, having $\geq 1$ previous infant death was associated with age $>35$ years (aOR1.6, 95\%CI1.0-2.7, $\mathrm{p}=0.05$ ), HIV diagnosis prior to index pregnancy (aOR 2.5, 95\%CI1.64.0, $\mathrm{p}<0.001)$ and 'very sick' self-report (aOR 10.1, 95\%CI 2.5$40.9, \mathrm{p}<0.001)$. Additionally, having a death of a child aged 1-5 was associated with age $>35$ (aOR 1.9, 95\% 1.2-2.7, $\mathrm{p}<0.001$ ), no formal education ( $\mathrm{aOR} 1.9,95 \% \mathrm{CI} 1.2-3.2, \mathrm{p}=0.01$ ), and being 'very sick' (aOR 5.2, 95\%CI1.3-20.5, $\mathrm{p}=0.02$ ).

\section{Conclusions}

Fertility and lifetime burden of child mortality in this cohort were high and associated with HIV-infection duration/severity. Further studies should explore ways to improve child survival in multiparous women under Option B+ in Malawi.

\section{P038 Risk Factors for Delayed HIV Diagnosis Among People With TB Symptoms: A Cross Sectional Study of People With TB Symptoms Attending Primary Clinics In Malawi}

Lucky-Gift Ngwira $^{1}$, David D. Dowdy ${ }^{2}$, McEwen Khundi ${ }^{1}$, Austin Nkhoma ${ }^{1}$, Augustine T. Choko ${ }^{1,6}$, Simon Makombe ${ }^{3}$, Michael Murowa ${ }^{4}$, Grace L. Barnes ${ }^{5}$, Richard E. Chaisson ${ }^{5}$, Elizabeth L. Corbett ${ }^{1,6}$, Katherine Fielding ${ }^{6}$

1. Malawi-Liverpool-Wellcome Trust Clinical Research Programme, Blantyre, Malawi

2. Department of Epidemiology, Johns Hopkins Bloomberg School of Public Health, Baltimore, Maryland, USA

3. HIV Department, Ministry of Health, Lilongwe, Malawi

4. Thyolo District Hospital, Thyolo, Malawi

5. Johns Hopkins School of Medicine, Baltimore, Maryland, USA

6. London School of Hygiene and Tropical Medicine, London, United Kingdom

\section{Introduction}

HIV and TB are closely related epidemiologically and clinically. MMJ VOL 28 (4): December 2016 
We investigated time to HIV diagnosis as a proxy for TB health seeking in adults newly diagnosed with HIV. Delay reporting of TB symptoms was defined as $>30$ days from debut of TB symptom to HIV diagnosis.

\section{Methods}

Newly diagnosed HIV positive adults ( $\geq 18 \mathrm{yrs}$ ) were asked about presence and duration of TB symptoms on enrolment from 10 rural primary clinics, as part of a cluster randomised trial. Considered risk factors for delayed health seeking included wealth score, age, gender by smoking status and time to clinic. Associations with delayed health seeking (>30dys) were evaluated using logistic regression

\section{Results}

TB symptoms were reported by 416/1,265 (32.9\%) of participants. Overall, 36.1\% (150/416) of participants with TB symptoms at recruitment had been symptomatic for $>30$ days. Most of the participants (62.5\%) were below the poverty line. After controlling for potential confounders, men (both smokers and non-smokers) were associated with higher odds of delayed health seeking, but this was not significant. Wealth, age and time taken to get to clinic from home were not significantly associated with delay.

\section{Conclusions}

Delayed diagnosis of HIV was common. No obvious effect of wealth in this study was observed but levels of poverty were strikingly common and severe in this population, with $62.5 \%$ fully below the 1998 poverty data line of a proxy means test. The findings would suggest that time to HIV diagnosis from onset of TB symptoms is readily obtainable and may inform on $90-90-90$ UNAIDS goals.

\section{P039 Why I keep at it: A qualitative study of women retained in Malawi's Option B+ program}

\author{
Nozgechi Phiri ${ }^{1}$, Andreas D. Haas ${ }^{1}$, Malango.T. Msukwa ${ }^{1,2}$, \\ Joep J. van Oosterhout ${ }^{3,4}$, Kali Tal ${ }^{1}$, Lyson Tenthani ${ }^{1,5}$, Hannock \\ Tweya $^{1,6,7}$, Olivia Keiser ${ }^{1}$ \\ 1. Institute of Social \& Preventive Medicine, University of Bern, Bern, \\ Switzerland \\ 2. The Baobab Health Trust, Lilongwe, Malawi \\ 3. Dignitas International, Zomba, Malawi \\ 4. Department of Medicine, College of Medicine, University of Malawi, \\ Blantyre, Malawi \\ 5. International Training \& Education Center for Health Malawi, \\ Lilongwe, Malawi \\ 6. Lighthouse Trust, Lilongwe, Malawi \\ 7. The International Union Against Tuberculosis and Lung Disease, \\ Paris, France
}

\section{Introduction}

Several studies have focused on barriers to uptake, retention and adherence in Malawi's Option B+ program, but only few studies have explored why women continue on ART.

\section{Methods}

We purposively selected pregnant and lactating women who were retained in Option $\mathrm{B}+$ for more than six months. Women $(\mathrm{n}=76)$ were recruited from 3 urban and 9 rural health facilities in Malawi. From November 2014 to December 2015, we conducted semistructured interviews $(\mathrm{N}=20)$ and focus group discussions $(\mathrm{N}=56)$ and performed a thematic analysis.
Results

From both data sources, we identified three major reasons why women continue ART: 1) desire to keep themselves healthy $(\mathrm{N}=25)$ and to protect their children from HIV infection $(\mathrm{N}=21)$; 2) partners $(\mathrm{N}=24)$, family or friends $(\mathrm{N}=25)$ encouraged them to continue treatment; 3) ART counselling helped them get through side effects $(\mathrm{N}=22)$. The surmounted obstacles to treatment, including social stigma, side effects, transport costs and verbal and physical harassment by health care workers.

\section{Conclusions}

The women in our study made informed treatment decisions and were able to overcome cultural norms, community, and facility barriers to continuing ART. In the absence of insurmountable economic barriers, improving ART counselling and increasing social support for HIV-positive women could improve retention and adherence. Programs that for example, strengthen follow up counselling by community health workers, peer support groups or campaigns designed to encourage disclosure and engage partners and family in ART.

\section{P040 We are offering HIV testing in HIV-exposed infants, not early infant diagnosis}

\section{Chikondi Malamba, Victor Mwapasa, Kenneth M. Maleta}

School of Public Health and Family Medicine, College of Medicine, University of Malawi, Blantyre, Malawi

\section{Introduction}

EID in HIV exposed infants and ART in infants is critical. In 2014 only $36-53 \%$ of infants less than 2 months who had a positive HIV results or diagnosed with severe HIV disease in Malawi were initiated on ART (1). In Malawi HIV testing for infants is centralized

\section{Methods}

Both quantitative and qualitative data collection methods were used. EID $\log$ books were reviewed and also structured questionnaires were administered to HIV positive mothers. Focus Group Discussions (FGDs) were conducted with Health workers and the HIV positive mothers with infants in Mangochi and Salima districts

\section{Results}

Turnaround time for the results was 63days (IQR 42 to 92) from Central Laboratories to Health Centres and 9 days (IQR 2 to 48) from the Health Centres to mothers and or guardians. From FGDs there identified were inefficient sample transportation, poor DBS tracking, stigma and discrimination and lack of community knowledge on EID and infants ART. Possible solutions to the stated problem were decentralization of HIV DNA PCR testing, conducting EID community awareness, introduction of Mother Infant Pairs (MIP) clinics and drones (Unmanned vehicles).

\section{Conclusions}

HIV DNA PCR test to HEI has to be at 6 to 8 weeks of age as recommended by WHO. This is not so in Malawi, many HEI attained this test late in 2014. Therefore there is need to increase the EID uptake, coverage and linkage to HIV care and treatment amongst the HIV exposed infants in Malawi. 